
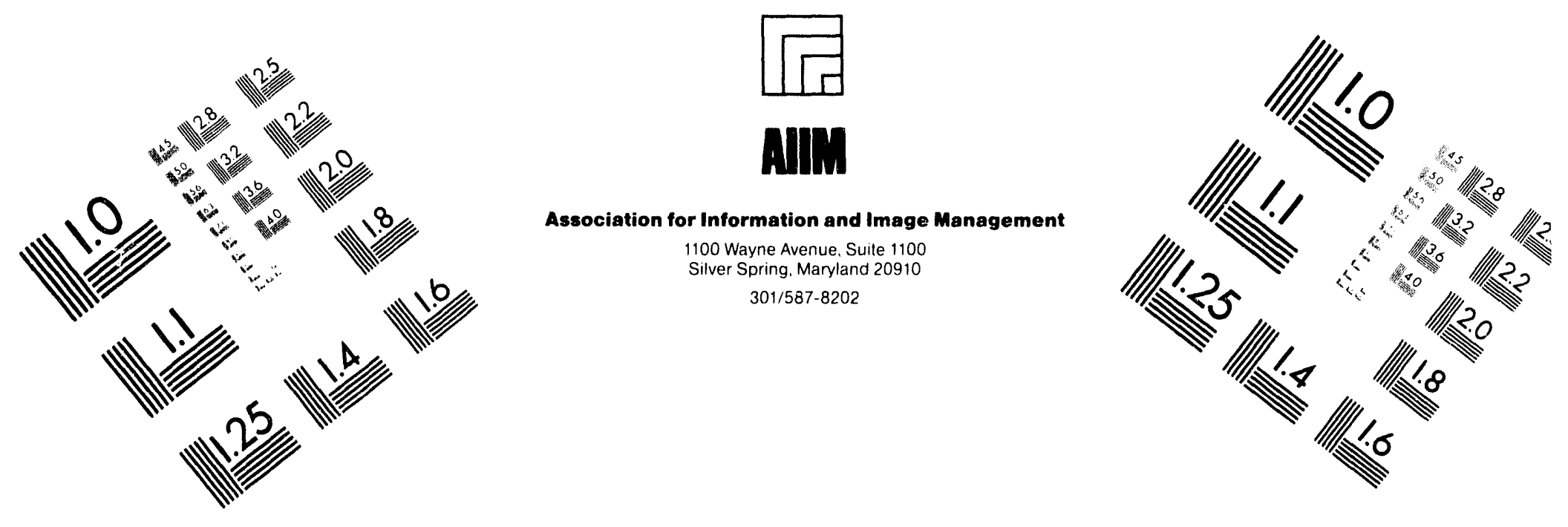

\title{
Centimeter
}
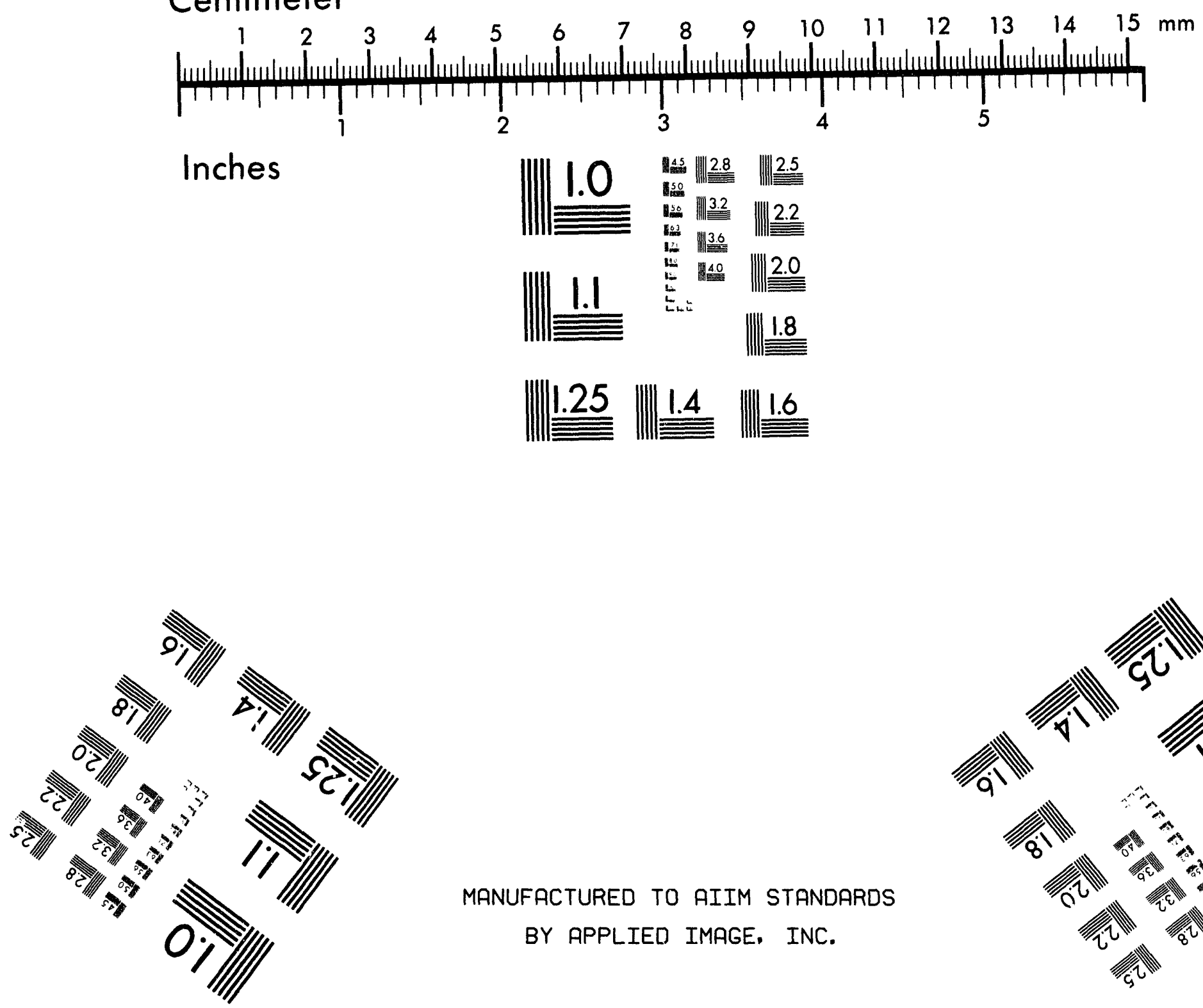

MANUFACTURED TO AIIM STANDARDS BY APPLIED IMAGE, INC.

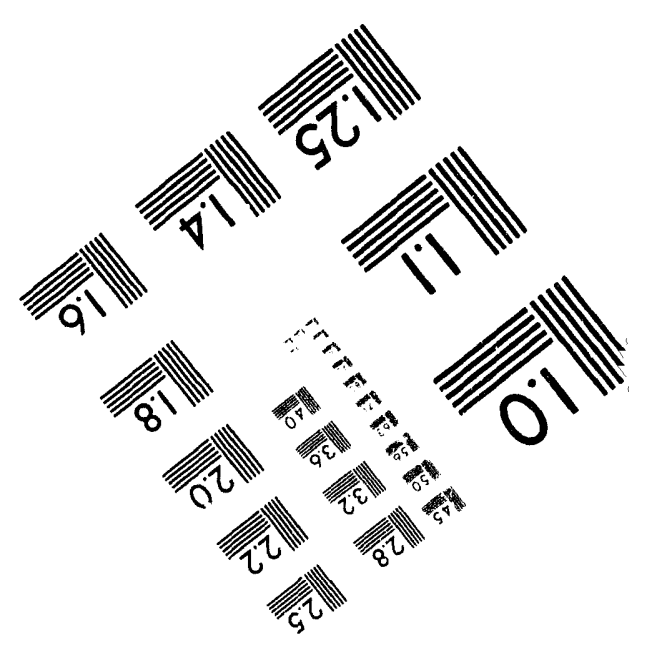



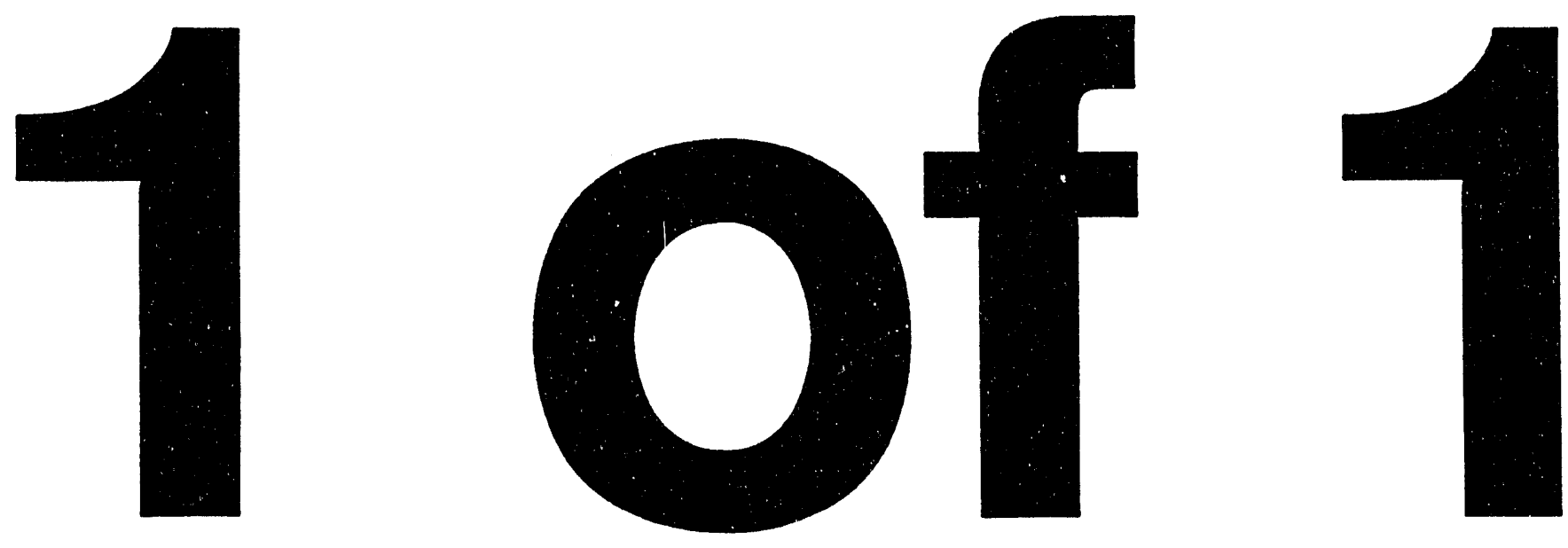


\section{Modeling Field Scale Unsaturated Flow and Transport Processes}

Manuscript Completed: June 1994

Date Published: August 1994

Prepared by

L.W. Gelhar, M.A. Celia, D. McLaughlin

T.J. Nicholson, NRC Project Manager

Department of Civil and Environmental Engineering Massachusetts Institute of Technology

77 Massachusetts Avenue

Cambridge, MA 02139

Prepared for

Division of Regulatory Applications

Office of Nuclear Regulatory Research

U.S. Nuclear Regulatory Commission

Washington, DC 20555-0001

NRC FIN D2044 


\begin{abstract}
A stochastic theory describing unsaturated flow and contamination transport in naturally hemerogeneous soils has been enhanced by adopting a more realistic characterization of soil variability. The enhanced theory is used to predict field-scale effective properties and variances of tension and moisture content. Applications illustrate the important effects of small-scale heterogeneity on large-scale anisotropy and hysteresis and demonstrate the feasibility of simulating two-dimensional flow systems at time and space scales of interest in radioactive waste disposal investigations.

Numerical algorithms for predicting field scale unsaturated flow and contaminant transport have been improved by requiring them to respect fundamental physical principles such as mass conservation. These algorithms are able to provide realistic simulations of systems with very dry initial conditions and high degrees of heterogeneity. Numerical simulation of the simultaneous movement of water and air in unsaturated soils has demonstrated the importance of air pathways for contaminant transport.

The stochastic flow and transport theory has been used to develop a systematic approach to performance assessment and site characterization. Prediction uncertainties have been quantified by considering the role of both natural heterogeneity and measurement error. Hypothesis-testing techniques have been used to determine whether model predictions are consistent with observed data.
\end{abstract}




\section{CONTENTS}



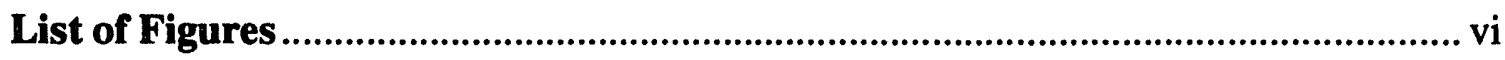

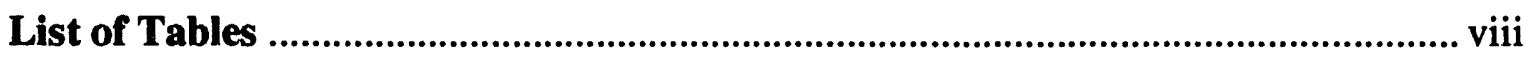

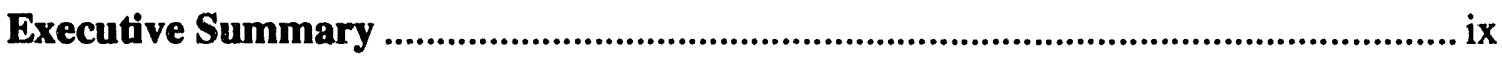

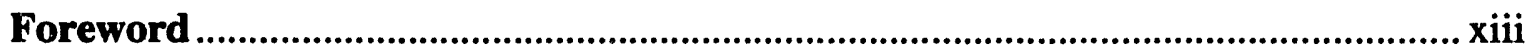

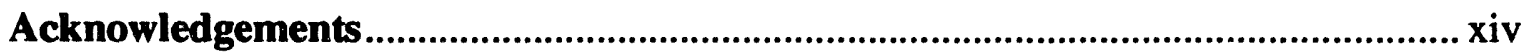

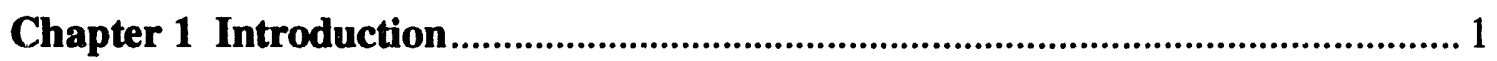

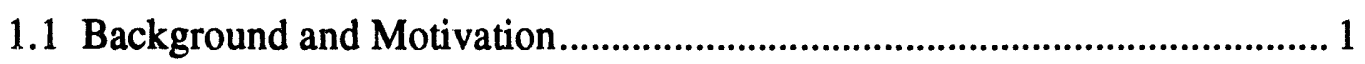

1.2 Report Scope and Content.......................................................................... 3

Chapter 2 Stochastic Theory of Unsaturated Flow and Transport........................ 5

2.1 Enhanced Stochastic Flow Theory ................................................................. 5

2.2 Applications of Stochastic Flow Theory ......................................................... 10

2.3 Stochastic Unsaturated Contaminant Transport ............................................... 15

Chapter 3 Numerical Modeling and Vapor Transport ........................................... 18

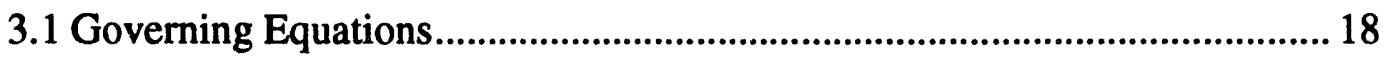

3.2 Numerical Methods for Richards Equation ................................................ 19

3.3 Inclusion of Air-Phase Dynamics .................................................................. 23

3.4 Modeling Contaminant Transport ............................................................... 26

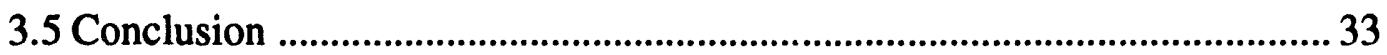

Chapter 4 Performance Assessment, Site Characterization and Model



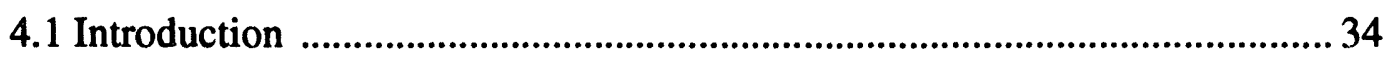

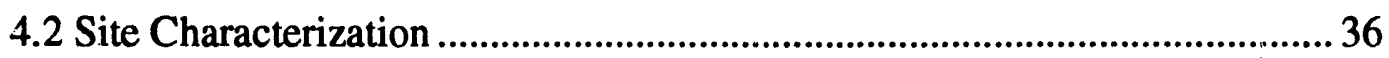

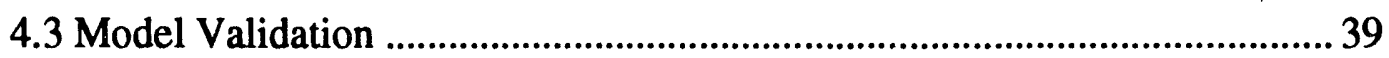

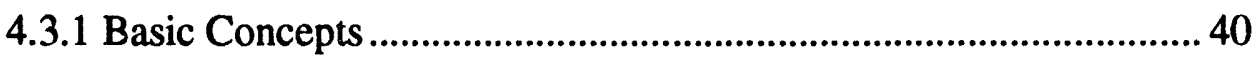

4.3.2 The Las Cruces Validation Study .................................................. 43

4.4 Uncertainty Evaluation ................................................................................... 55

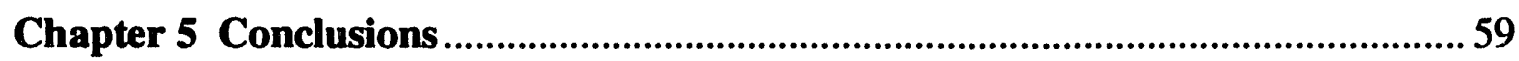

References 


\section{LIST OF FIGURES}

Page

Figure 1-1. Schematic illustration of subsurface transport processes and possible transport pathways found at a hypothetical low-level disposal site.

Figure 2-1. Unsaturated hydraulic conductivity versus capillary tension head for the Maddock sandy loam. Each curve corresponds to a different spatial location (from Polmann et al., 1988)

Figure 2-2. Logarithmic hydraulic conductivity curve illustrating the parameterization of equation [2.2].

Figure 2-3. Sensitivity of effective conductivity, anisotropy and tension variance to variations in a (from Polmann, 1990).

Figure 2-4. Contours of equal bromide concentration ( $¥ 10-5 \mathrm{~mol} / \mathrm{kg}$ soil) from hillslope tracer experiment on a sand dune (from McCord et al., 1991). 9

Figure 2-5. Detailed and mean simulation domains (from Polmann et al., 1991)....... 12

Figure 2-6. Tension distributions for the detailed and mean simulations at 15 days (from Polmann et al., 1991).

Figure 2-7. Vertical tension profiles under wetted strip centerline for the detailed and mean simulations (from Polmann et al., 1991)

Figure 2-8. Longitudinal macrodispersivity versus mean tension for Maddock soil under steady-state flow with uniform mean tension (from Polmann, 1990).

Figure 2-9. Longitudinal dispersivities for unsaturated soils as related to displacement scale (from Gelhar, 1992).

Figure 3-1. Numerical solutions using (a) h-based simulator and (b) mixed formulation (from Celia et al., 1990a).

Figure 3-2. Mass balance result for finite element and finite difference solutions to the h-based equation. Mixed formulation solutions produce mass balance ratios equal to one for all time step sizes (from Celia et al., 1990a).

Figure 3-3. Experimental setup for air-water example showing boundary conditions, including possible ponding. Darker shading indicates higher water content (from Celia and Binning, 1992a)

Figure 3-4. Time evolution of infiltrating water front (as shown in grey scale) and air movement (shown as particle trajectories) for column with open bottom and no ponding at the surface (from Celia and Binning, 1992a).

Figure 3-5. Schematic for contaminant transport simulations (from Sweed et al., 1992). 
Figure 3-6. Contamination transport only in the water phase. Concentration profiles are shown at 10 minute intervals.

Figure 3-7. Contaminant plume evolution in time with equilibrium partitioning between the water and air phases. Concentration in the air phase differs from the water-phase concentration by Henry's constant.

Figure 3-8. Concentration in the water phase with a kinetic mass transfer between the water and air phases.

Figure 3-9. Concentration in the air phase for kinetic mass transfer with initial condition $\mathrm{ca}=0$ and $\mathrm{cw}=1$ between $30-35 \mathrm{~cm}$ depth.

Figure 4-1. A clustered sampling strategy for site characterization (from Rajaram and McLaughlin, 1990). 38

Figure 4-2. Effective parameters for the deterministic model. 45

Figure 4-3a. Effective parameters for the stochastic model. 46

Figure 4-3b, c, d. Effective parameters for the stochastic model. 47

Figure 4-4. Las Cruces moisture content contours at 82 days. 49

Figure 4-5. Las Cruces moisture content contours at 737 days. 50

Figure 4-6. Las Cruces vertical transects of moisture content at 82 days...................... 51

Figure 4-7. Las Cruces vertical transects of moisture content at 737 days....................552

Figure 4-8. Margins of error for models with different prediction uncertainty ( $\mathrm{u}=$ exposure, $\mathrm{uT}=$ specified exposure threshold, $\hat{u}=$ model prediction). 56 


\section{LIST OF TABLES}

Page

TABLE 1 Hypothesis Tests Results Over All Locations at Specified Times (5\% Significance Level) ..............................................................................................5 54

TABLE 2 Hypothesis Tests Results at Outlying Boreholes at Specified Times (5\% Significance Level) 54

TABLE 3 Hypothesis Tests Results at Centerline Borehole at Specified Times (5\% Significance Level) 54 


\section{EXECUTIVE SUMMARY}

The scales of concern in subsurface transport of contaminants from low-level radioactive waste disposal facilities are in the range of 1 to $1000 \mathrm{~m}$. Natural geologic materials generally show very substantial spatial variability in hydraulic properties over this range of scales. Such heterogeneity can significantly influence the migration of contaminants. It is also envisioned that complex earth structures will be constructed to isolate the waste and minimize infiltration of water into the facility. The flow of water and possible contaminated gases tïrough such complex constructed facilities must also be a concern. This report focus on unsaturated flow and transport processes which are pertinent to fieldscale modeling for LLW facilities.

Detailed small-scale (say less than $10 \%$ of the overall problem scale) variations of unsaturated hydraulic properties are practically impossible to resolve in a realistic measurement program. Practical field-scale models at least implicitly assume some type of aggregated or smoothed "effective" parameters which presumably incorporate the influence of small-scale heterogeneity. The stochastic theory developed through this research provides an explicit method of predicting the effective large-scale parameters using practical small-scale measurements interpreted in a statistical framework.

Unsaturated flow theory, based on the description of soil heterogeneity in terms of threedimensional statistically anisotropic stationary random fields representing the imperfect horizontai iayering which is typical of sedimentary deposits, has produced new results quantitatively demonstrating the tension-dependent anisotropy in the large-scale effective hydraulic conductivity tensor as well as macro-hysteresis. The stochastic flow theory has been enhanced to realistically represent unsaturated hydraulic conductivity characteristics over a wide range of tensions, thus making it possible to treat the initially very dry soils which are frequently encountered in semi-arid settings. It was found that the degree of hydraulic anisotropy (ratio of horizontal to vertical conductivity) varies greatly and is typically most pronounced in dry (high tension) soils. The degree of anisotropy is very sensitive to the variability of the slope of the log-conductivity vs. tension curves; this fact emphasizes the importance of measuring unsaturated hydraulic conductivities if this anisotropy phenomenon is to be realistically represented. The stochastic approach also predicts the variance of tension or moisture content, and therefore can be used to assess the uncertainty of model predictions. Applications of the stochastic theory using advanced numerical techniques demonstrate the feasibility of simulating realistic fieldscale unsaturated flow problems over scales of tens of meters and periods of tens of years.

A stochastic treatment of transport of a nonreactive solute under unsaturated flow conditions shows that the macrodispersivity is influenced primarily by the variations in the unsaturated hydraulic conductivity with only a minor effect of the variation in moisture content. The theory indicates that the longitudinal macrodispersivity increases modestly with increasing tension (as the soil becomes drier). In the unsaturated case, the existing field experiments are not adequate to test the transport theory, but a summary of 
field observations shows that unsaturated longitudinal macrodispersivities are of the same order of magnitude as predicted by the theory.

The research also addressed the influence of soil heterogeneity on diffusive transport of gaseous contaminants. Using a stochastic treatment, it has been found that the effective diffusion coefficient becomes a tensorial property with only a modest degree of anisotropy for perfectly dry soils (at most a factor of 2) but as much as an order of magnitude decrease in the effective diffusivity in the direction perpendicular to bedding in the more realistic case of moist soils.

Because numerical simulation will play an important role in evaluating disposal facilities, robust and reliable numerical methods are required. Commonly used numerical approaches are often inadequate to solve certain types of problems that are relevant to disposal facilities, such as initially dry soils in arid regions. The research performed under this contract resulted in a clear explanation of why numerical difficulties occur in standard simulators. It also presented an alternate approach that alleviates the problems found in standard approaches. The new methodology was shown to be reliable and accurate over a wide range of soil conditions. Coupled with specific conjugate gradient algorithms for matrix solution, the method provides efficient and robust simulations of infiltration events in initially very dry and highly heterogeneous soils.

The numerical approach described above for the Richards equation has been extended and applied to the case of the simultaneous movement of air and water in unsaturated soils. Such a simulator provides quantitative estimates of both water and air fluxes, from which potential exposures can be assessed. These simulations also provide guidance about when Richards equation is a good approximation for water flow. In addition, it points out that the solution of Richards equation provides no information about air movement, so that if vapor transport is of concern, Richards equation may be inappropriate under any circumstance. If advective transport of gaseous contaminants is a concern, then a two-phase simulation of both air and water is appropriate.

Incorporation of contaminant transport simulators into the two-phase flow simulator provides further insights into contaminant movement in unsaturated soils. The partitioning of a contaminant between the air and water phases, and the rate at which this partitioning takes place, are very important considerations when considering vapor transport. This will indicate whether, or in what amounts, a contaminant will migrate in the air phase, potentially towards the land surface. It also highlights the importance that source-term characterization plays in unsaturated-zone simulations.

Even the best models of unsaturated flow and transport cannot be expected to field perfect predictions. Model prediction accuracy is compromised by natural heterogeneity, uncertain external disturbances, and data limitations. A complete performance assessment should consider all of these factors in an integrated way. It is convenient to distinguish three closely related aspects of performance assessment: site characterization, model validation, and uncertainty evaluation. Site characterization provides the field information needed to construct and test predictive models. Model validation checks that predictions 
are consistent with field observations. Uncertainty evaluation provides quantitative estimates of prediction accuracy over the time and space scales of interest in performance assessment.

A LLW performance assessment is no better than the site characterization on which it depends. The characterization should be designed, as much as possible, to identify the physical and chemical processes that affect waste transport at the site. In particular, the characterization should provide information about large-scale hydrogeologic features and climatic fluctuations as well as information about small-scale variability. If small-scale variability is characterized statistically, a special effort should be made to collect the data needed to estimate effective parameters and small-scale variances. Clustered sampling provides an efficient and cost-effective way to characterize multi-scale variability. Although general guidelines can be helpful, characterization efforts should always be tailored to the unique features of each site.

Most methods for evaluating prediction accuracy assume that the model in question is structurally correct -- i.e., that it is able to provide perfect large-scale predictions for some set of physically plausible inputs. When structural errors are negligible, prediction errors can be attributed either to small-scale variability or to input errors. In classical statistics, the primary objective of model validation is to test for structural errors. If a model is not rejected by the validation procedure, it may be used to derive long-term accuracy estimates which are conditioned on the hypothesis that structural errors are negligible. This classical approach to model validation can be extended to unsaturated flow and transport models. The key is to derive the statistical properties of small-scale variability and input error from a stochastic theory which explicitly accounts for natural heterogeneity. An application of stochastic concepts to the Las Cruces, New Mexico infiltration experiment indicates that classical hypothesis testing methods can provide a rigorous basis for model validation. Hypothesis tests are also able to identify the strengths and weaknesses of models which rely on simplifying assumptions and limited field data.

The methods currently available for analyzing the long-term predictive accuracy of conditionally validated models rely on many simplifying assumptions and/or are computationally demanding. For these reasons, practical site performance analyses have to be based on simplified models which focus on a few key transport processes. These models will probably achieve computational efficiency by sacrificing temporal and spatial resolution. Future performance assessment research should recognize that a quantitative evaluation of predictive uncertainty is needed to determine whether there is a "reasonable expectation" that a candidate site can meet LLW safety requirements. Uncertainty evaluation should also play a prominent role in LLW performance assessment guidelines and licensing regulations. 


\section{FOREWORD}

This technical report was prepared by Massachusetts Institute of Technology (MIT) under their research project with the Waste Management Branch in the Office of Nuclear Regulatory Research (FIN D2044). The report presents information on improved methods for predicting field-scale contaminant transport in heterogeneous unsaturated soils relevant to near-future low-level radioactive waste (LLW) disposal. In particular, recent enhancements and applications of MIT's stochastic theory and methods are documented and demonstrated. This report also discusses research results in the topical areas of numerical modeling, vapor transport, performance assessment, site characterization and model validation related to LLW.

NUREG/CR-5965 is not a substitute for NRC regulations, and compliance is not required. The approaches and/or methods described in this NUREG/CR are provided for information only. Publication of this report does not necessarily constitute NRC approval or agreement with the information contained herein. 


\section{ACKNOWLEDGEMENTS}

The research described in this report is part of a continuing study of flow and contaminant transport in heterogeneous unsaturated soils supported by the U.S. Nuclear Regulatory Commission (USNRC) through the research project entitled "Improved Methods for Predicting Field-Scale Contaminant Transport in Heterogeneous Unsaturated Soils" (Contract No. NRC-04-88-074).

Thomas Nicholson of the USNRC served as Project Manager for the project and contributed significantly to this work through his enthusiastic support of the MIT research and the cooperative work on the field experiment at New Mexico State University (NMSU) in Las Cruces, NM. Tom raised many important questions and inspired considerable thought and discussion during lively meetings.

Peter J. Wierenga, now at the University of Arizona, was responsible for developing the Las Cruces field experiment and provided the field data used in the model validation work; his suggestions and cooperation are gratefully acknowledged. Glendon Gee of Pacific Northwest Labs also provided useful insights on modeling and interpretation of experiments. 


\section{CHAPTER 1}

\section{INTRODUCTION}

\subsection{Background and Motivation}

The U.S. Nuclear Regulatory Commission (USNRC) has been directed by Congress in the Low-Level Radioactive Waste Policy Amendments Act of 1985 to develop regulatory guidance and to assist the states in their responsibilities of siting, assessing and licensing future low-level radioactive waste (LLW) disposal facilities. General performance objectives and technical requirements for such facilities are outlined in Subparts C and D of 10 CFR Part 61 (Dffice of the Federal Register, 1987) and discussed in more detail in NUREG-1200, the USNRC's Standard Review Plan (USNRC, 1991). Both of these documents emphasize near-surface land disposal in the unsaturated zone. Subpart $C$ of 10 CFR Part 61 limits the release of radioactive materials "which may be released to the general environment in ground water, surface water, air, soil, plants and animals", and Subpart D states that near-surface sites "shall be capable of being characterized, modeled, analyzed and monitored". These requirements form the basis for the extensive discussion of site meteorology, geology, and hydrclogy presented in NUREG-1200.

Subsurface hydrology is a particularly important aspect of site characterization because subsurface water and vapor transport are the primary natural pathways for the movement of contaminants from a disposal facility to the accessible environment. Such transport may move in any direction -- down to the water table, laterally into adjacent soils and root systems, or upward towards the atmosphere. This is illustrated in a schematic way in Figure 1-1, which shows some of the subsurface transport processes which may operate at a near surface site. A site analysis should consider all of these processes, if it is to satisfy the broad requirements of 10 CFR 61, Part C.

Analysis of subsurface contaminant transport is complicated by a number of factors. These include the difficulty of observing subsurface processes, the long-time scales required for experiments, and the need to account for soil-water interactions and spatial variability. The situation is particularly complex in the unsaturated zone, where soil properties change dramatically with moisture content. Since spatial variability and nonlinear behavior greatly complicate the equations which describe unsaturated flow and transport, performance assessments for unsaturated sites must rely heavily on numerical models. This is explicitly acknowledged in the ground-water characterization section of NUREG-1200. It is expected that subsurface flow and transport modeling will play a major role in performance assessment and safety analyses for LLW facilities.

This report summarizes recent enhancements and applications of MIT's stochastic approach for modeling contaminant transport in heterogeneous unsaturated soils which were developed through the USNRC research project entitled "Improved Methods for Predicting Field-Scale Contaminant Transport in Heterogeneous Unsaturated Soils" (Contract No. NRC-04-88-074). 


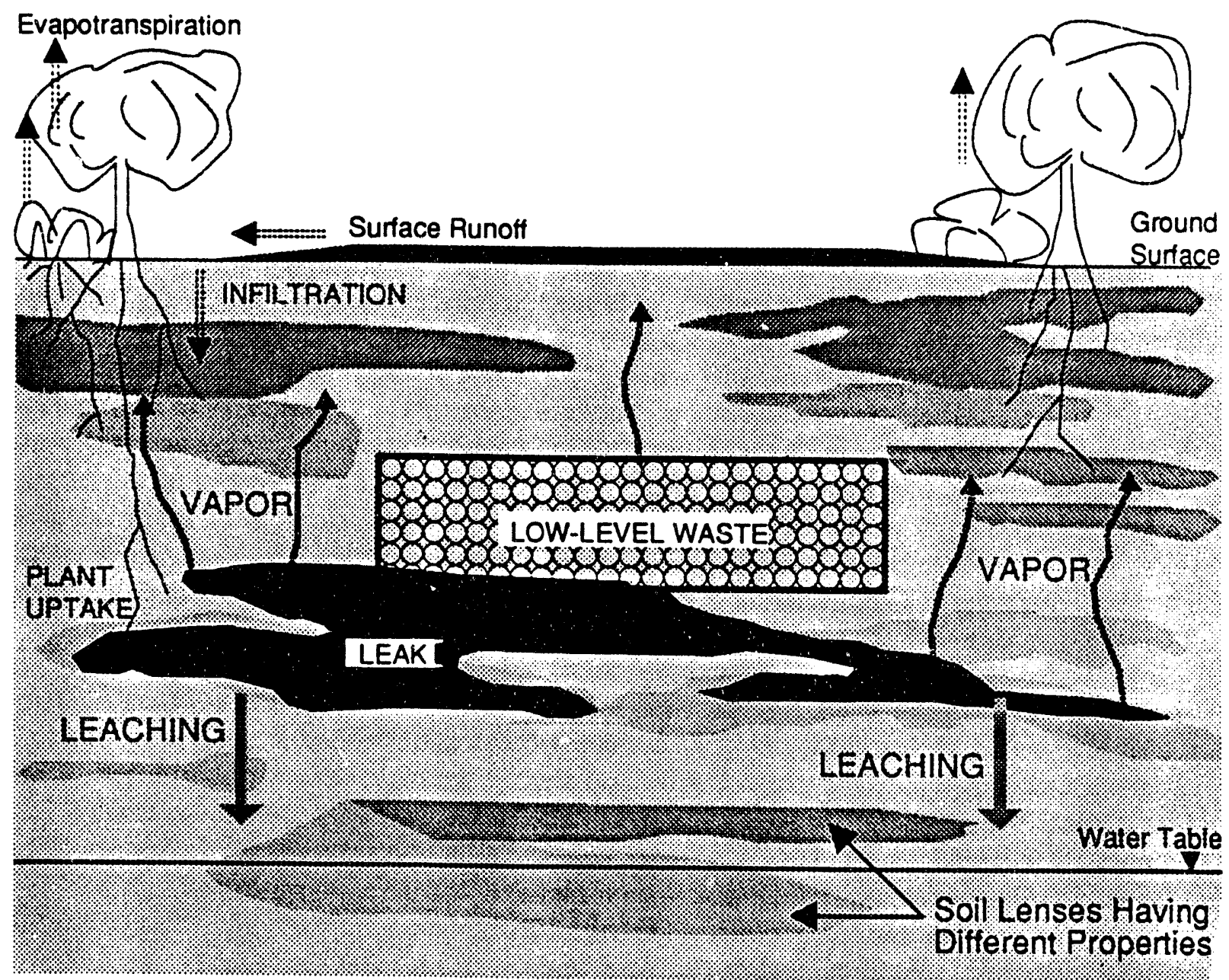

Figure 1-1. Schematic illustration of subsurface transport processes and possible transport pathways found at a hypothetical low-level disposal site. 
The MIT stochastic approach to unsaturated flow and transport has a number of important advantages. It is able to represent natural variability with a relatively small number of parameters which can be estimated from field observations. It yields relatively simple expressions for the "effective" soil properties which influence the large-scale behavior of contaminant plumes. Numerical models based on such effective parameters can be used to analyze waste migration in situations where data limitations make conventional deterministic modeling impractical. Finally, the stochastic approach explicitly acknowledges the uncertainty associated with predictions of subsurface contaminant transport. This makes it particularly compatible with a broader analysis of exposure risk.

Although MIT's stochastic theory is relatively new, it already promises to change the way hydrologists and soil physicists think about unsaturated flow and transport at the field scale. Traditional deterministic models of the unsaturated zone cannot account for the highly variable soil conditions found in natural environments unless they are provided with enormous amounts of data. A detailed three-dimensional characterization of a site having dimensions on the order of 50 by 50 by 10 meters could easily require tens of thousands of soil samples (Ababou et al, 1988). Since this is impractical, most deterministic models rely on highly aggregated descriptions which cannot account for the spreading and dispersion caused by small-scale heterogeneities.

It is important to note that small-scale fluctuations in soil properties are important because they have large-scale consequences (e.g., anisotropy and macrodispersion). On thc other hand, small-scale fluctuations in moisture content or concentration may not be particularly important, at least from a licensing point-of-view. Stochastic methods capitalize on this contrast by treating small-scale soil property fluctuations as if they were random and then focusing on mean (i.e., large-scale) moisture and solute behavior. In this way, the large-scale consequences of local variability are incorporated in the analysis without requiring a detailed characterization of individual soil layers and anomalies. This is why the stochastic approach can provide such an efficient characterization of heterogeneous real-world sites.

\subsection{Report Scope and Content}

Earlier developments of the MIT stochastic approach (Polmann et al., 1988) demonstrated the possibility of simulating field-scale unsaturated flows using stochastically-derived effective parameters, but pointed to the need for enhancements of the theory to treat more realistic soil characteristics and improvements in numerical techniques to permit simulations over space scales and times of practical interest. That report also emphasized the need to investigate the implications of the stochastic approach for model validation, site characterization and performance assessment. The purpose of this report is to summarize the key findings of this research which focused on these unresolved issues. The report covers only the important highlights of the research and their practical implications; full technical details are available in several theses, reports and journal articles as cited herein. 
Chapter 2 of the report outlines the important enhancements of the stochastic flow theory and illustrates some applications of the theoretical results using simple analytical expressions and advanced numerical simulation techniques. The use of the enhanced flow theory to derive more general results for field-scale transport of contaminants in liquid and gaseous phases is also summarized. Discussions emphasize the applied implications of the new results and the important limitations of current scientific understanding of the pertinent processes.

Chapter 3 outlines the numerical procedures that have been developed in this research, explaining why certain approximation methods are better than others. The results of this analysis, which was based on the Richards equation, are then expanded and applied to the case of two-phase flow, involving the simultaneous movement of air and water in the unsaturated zone. Contaminant transport is included in each phase, with both equilibrium and nonequilibrium partitioning of the contaminant included. Numerical simulations are used to demonstrate the potential importance of the air pathways. These simulations serve as examples of the procedure by which quantitative estimates of contaminant fluxes in both the water and vapor phases may be obtained.

Chapter 4 considers how predictive models, both stochastic and deterministic, can be used to evaluate site performance in realistic situations where input data are limited and uncertain. In such situations, performance assessment should account for the fact that long-term model predictions will be imperfect approximations. 'The accuracy of these predictions depends upon the heterogeneity of the site, the nature of external disturbances and data available for site characterization. Chapter 4 presents a stochastic approach to performance evaluation which integrates all of these factors in a systematic way. This approach suggests some general guidelines for site characterization and indicates how model validation fits into the overall performance assessment procedure. A new hypothesis-testing approach to model validation is proposed and illustrated with a field application. Chapter 4 ends with a discussion of the factors which affect the uncertainty of long-term predictions obtained from validated models.

Chapter 5 provides general conclusions as to the major findings and implications of the research. In particular, potential applications of the earlier detailed improved methods developed in this project for LLW sites are briefly discussed. 


\section{CHAPTER 2}

\section{STOCHASTIC THEORY OF UNSATURATED FLOW AND TRANSPORT}

\subsection{Enhanced Stochastic Flow Theory}

The unsaturated hydraulic characteristics curves for natural soils tend to be more complex than the simple analytical forms which are commonly used. Earlier developments of stochastic models of unsaturated flow (Yeh et al., 1985c; Mantoglou and Gelhar, 1987c) were based on such simplified representations of the hydraulic characteristics and experience in applying those theories in field situations with initially very dry soils (e.g., Polmann et al., 1988) indicated that more general representations would be required to realistically simulate a wide range of field conditions. Therefore, as part of the project work, the stochastic theory has been enhanced to provide greater realism and applications flexibility. The approach used to enhance the stochastic theory is summarized below, focusing on the representation of the hydraulic conductivity. Compicte details on the eni..nncements of the theory are available in Polmann (1990).

A frequently used form for the unsaturated hydraulic conductivity is

$$
K(\psi)=K_{s} e^{-\alpha \psi}
$$

where $\psi$ is the tension, $K_{S}$ is the saturated conductivity and $\alpha$ is a parameter representing the slope of the $\ln K$ vs. $\psi$ graph. Figure 2-1 shows the unsaturated hydraulic conductivity curves observed at several different locations in a field of Maddock sandy loam. This graph illustrates the variability of unsaturated hydraulic conductivity and shows that $\ln K$ decreases in a roughly linear fashion with increasing tension as represented by [2.1] with $K_{S}$ and $\alpha$ as constant parameters. In order to represent the modest nonlinearity of the individual curves in Figure 2-1, [2.1] is generalized as follows:

$$
\ln K=F_{0}(\psi)-\alpha(\psi) \psi
$$

Here $\alpha(\psi)$ and $F_{O}(\psi)$ are slowly varying functions of tension which represents the local slope and corresponding intercept of an individual curve at a given tension (see Figure 22). Using this generalized representation, the parameters $\alpha$ and $F_{O}$ can be evaluated as a function of tension for several different soil samples. From those data the mean and variation about the mean are then determined as a function of tension. In general the variations about the mean will vary systematically with mean tension so that normalizing functions are introduced to produce scaled variations which are independent of tension. The cross-correlation between the normalized fluctuations of $\alpha$ and $F_{O}$ is also evaluated using this approach. Polmann (1990) demonstrates the application of this characterization approach using the data for the Maddock soil (Figure 2-1).

The generalized soil characterization outlined above then serves as the input to the stochastic analysis which treats the fluctuations of input parameters and the tension as locally stationary statistically anisotropic random fields. These fluctuations are introduced 


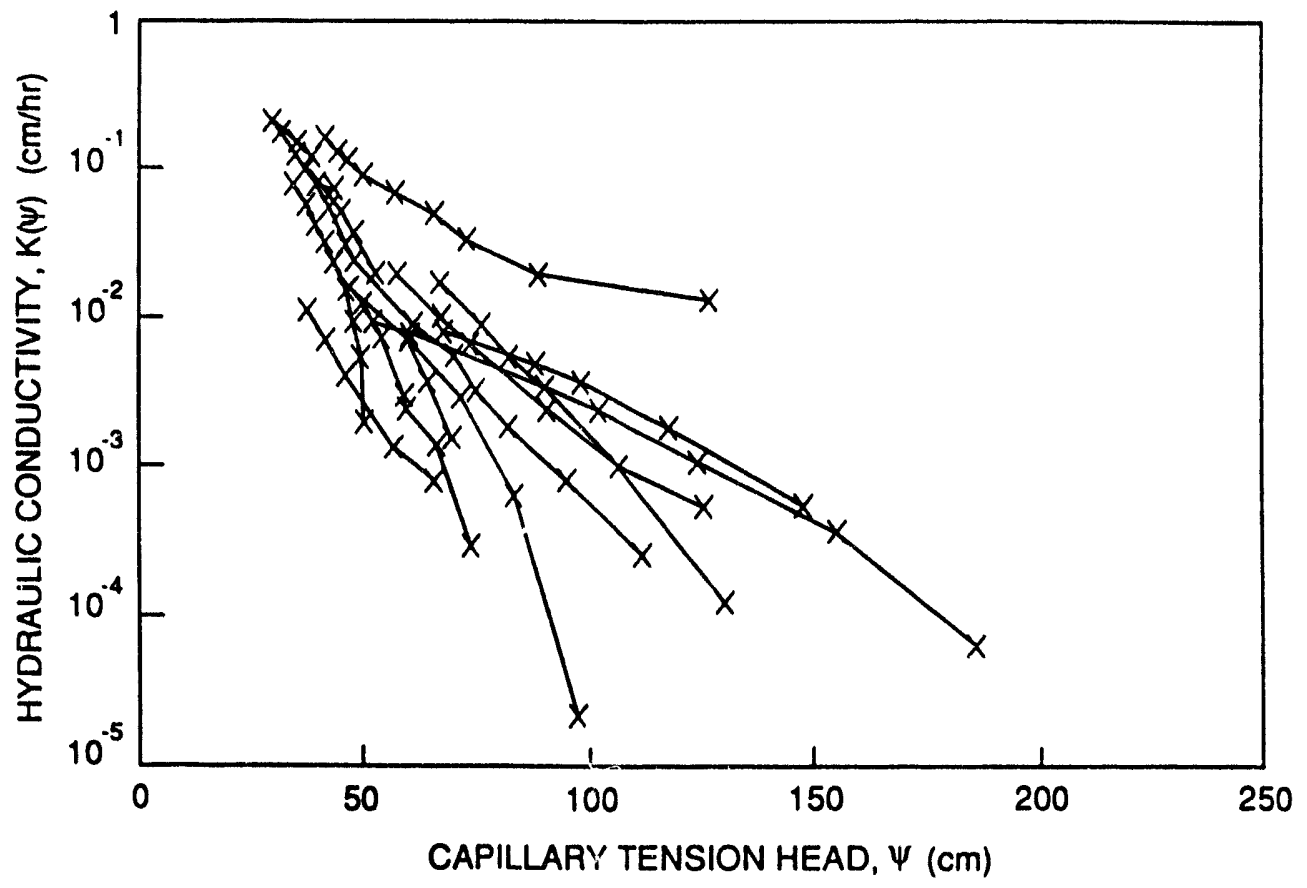

Figure 2-1. Unsaturated hydraulic conductivity versus capillary tension head for the Maddock sandy loam. Each curve corresponds to a different spatial location (from Polmann et al., 1988)

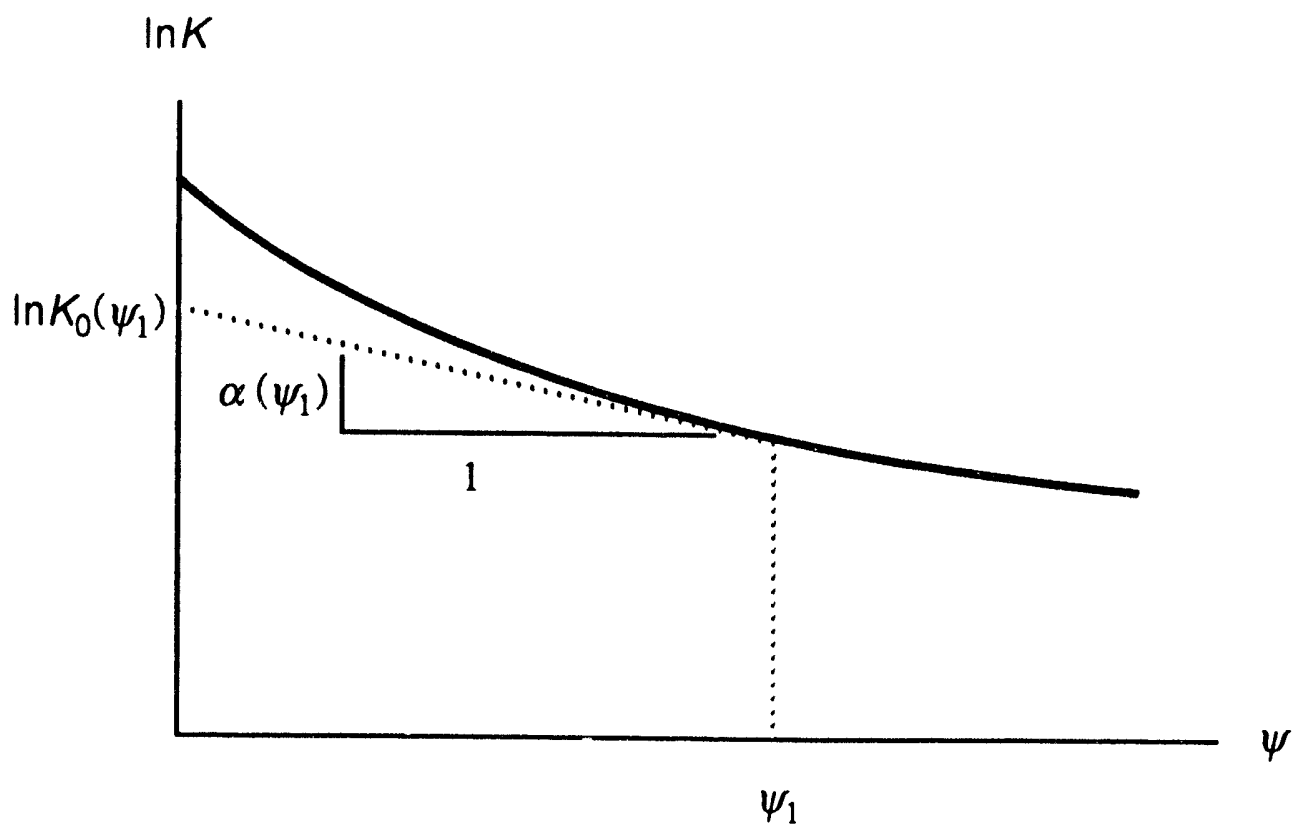

Figure 2-2. Logarithmic hydraulic conductivity curve illustrating the parameterization of equation [2.2]. 
in the unsaturated flow equation and the resulting stochastic differential equation is solved analytically by spectral methods under the assumption of relatively small fluctuations. The resulting solution is used to evaluate the effective large-scale parameters which appear in the mean flow equation and the variance of the predicted variables (tension and moisture content).

The results of the enhanced stochastic unsaturated flow analysis are expressed in terms of a general system of simultaneous nonlinear algebraic equations which determine the effective hydraulic conductivity tensor and the tension variance (see Polmann, 1990, Table 3-1). General expressions for the effective conductivity and the tension variance can not be found explicitly but rather depend on the spatial and temporal derivatives of the mean tension which in turn depend on the effective conductivity through the governing mean equation. In this general case the mean flow equation must be solved simultaneously with the effective conductivity relationships using numerical methods. The effective conductivity generally exhibits tension-dependent anisotropy and hysteresis.

For the special case of steady uniform vertical infiltration, for which the temporal and spatial derivatives of mean tension are null, explicit results for the effective conductivity and tension variance can be found (see Polmann, 1990, Table 3-2 and equations 3-161 through 3-163). Some results for Maddock soil with steady infiltration are illustrated in Figure 2-3 which shows degree of anisotropy of the effective hydraulic conductivity tensor and the head variance as a function of mean tension; also shown is the sensitivity of the results to changes in the variance of the normalized residual of the $\alpha$ fluctuations by factors of 2 . The results are generally found to be most sensitive to the degree of variability of the slope of the $\ln K-\psi$ curve, but are also importantly influenced by the correlation between $\alpha$ and $F_{o}$.

The enhanced theory also takes into account the effects of nonlinear moisture retention characteristic curves as illustrated by Polmann (1990). Mantoglou (1992) has also proposed a somewhat different method of incorporating the influence of nonlinear soil characteristic curves.

The enhancements of the stochastic theory are found to be very significant in terms of the influence on the degree of anisotropy of the effective conductivity. For the Maddock soil, if the log-linear form, [2.1], is used assuming uncorrelated $K_{S}$ and $\alpha$, the calculated anisotropy ratio at $200 \mathrm{~cm}$ of mean tension is around 1011 (see Polmann, 1990), whereas the enhanced theory produces a ratio of 4 (Figure 2-3). The classical log-linear results will become even more unrealistic when a wider range of tension is involved. Overall, the enhanced theory demonstrates the sensitivity of the large-scale effective conductivity to the variability of unsaturated hydraulic conductivity, thereby emphasizing the importance of making direct measurements of unsaturated hydraulic conductivity. Extrapolations based on only saturated conductivity measurements (e.g. van Genuchten, 1980) are not likely to realistically capture these important effects. 

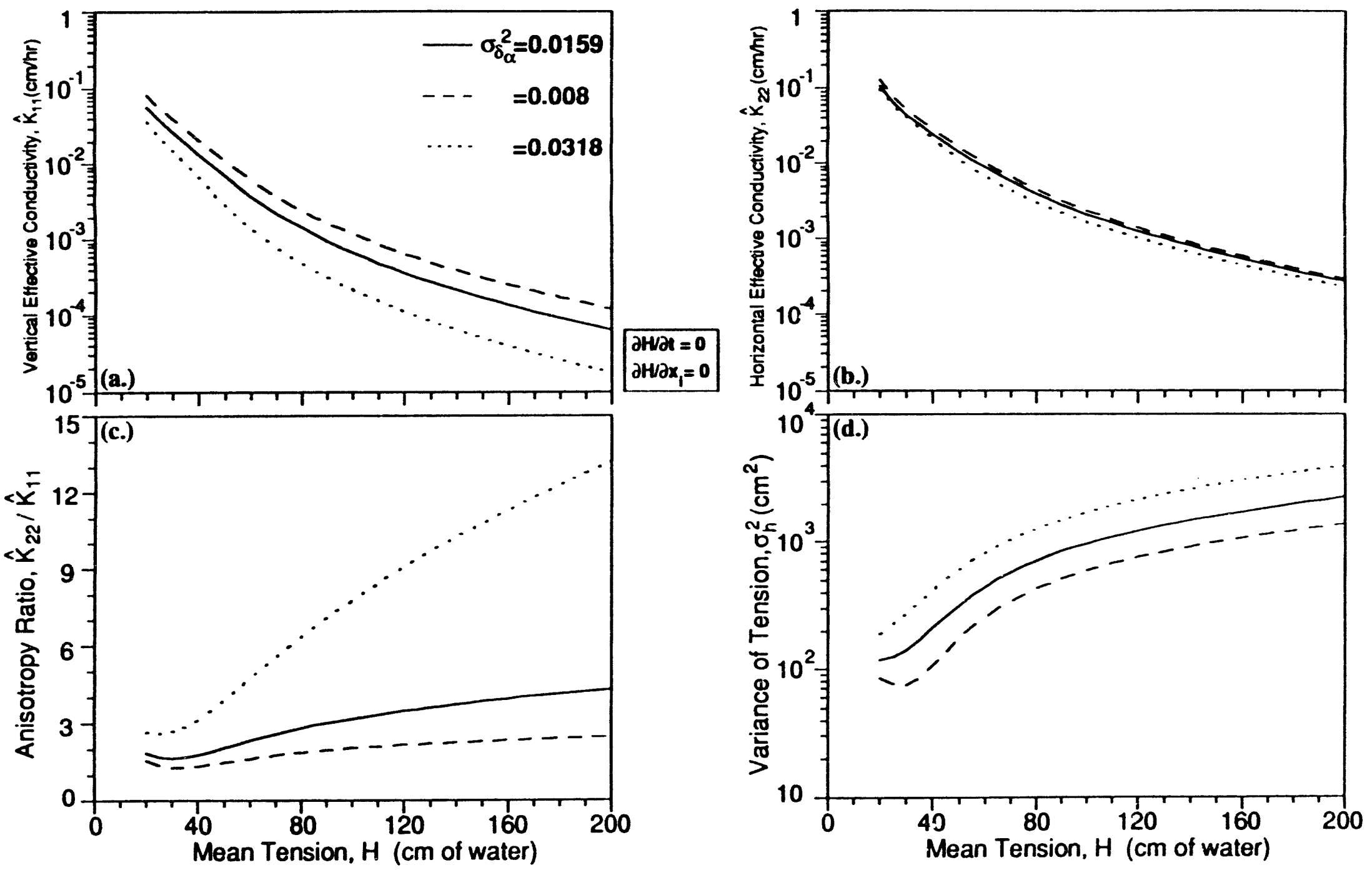

Figure 2-3. Sensitivity of effective conductivity, anisotropy and tension variance to variations in $\alpha$ (from Polmann, 1990), 


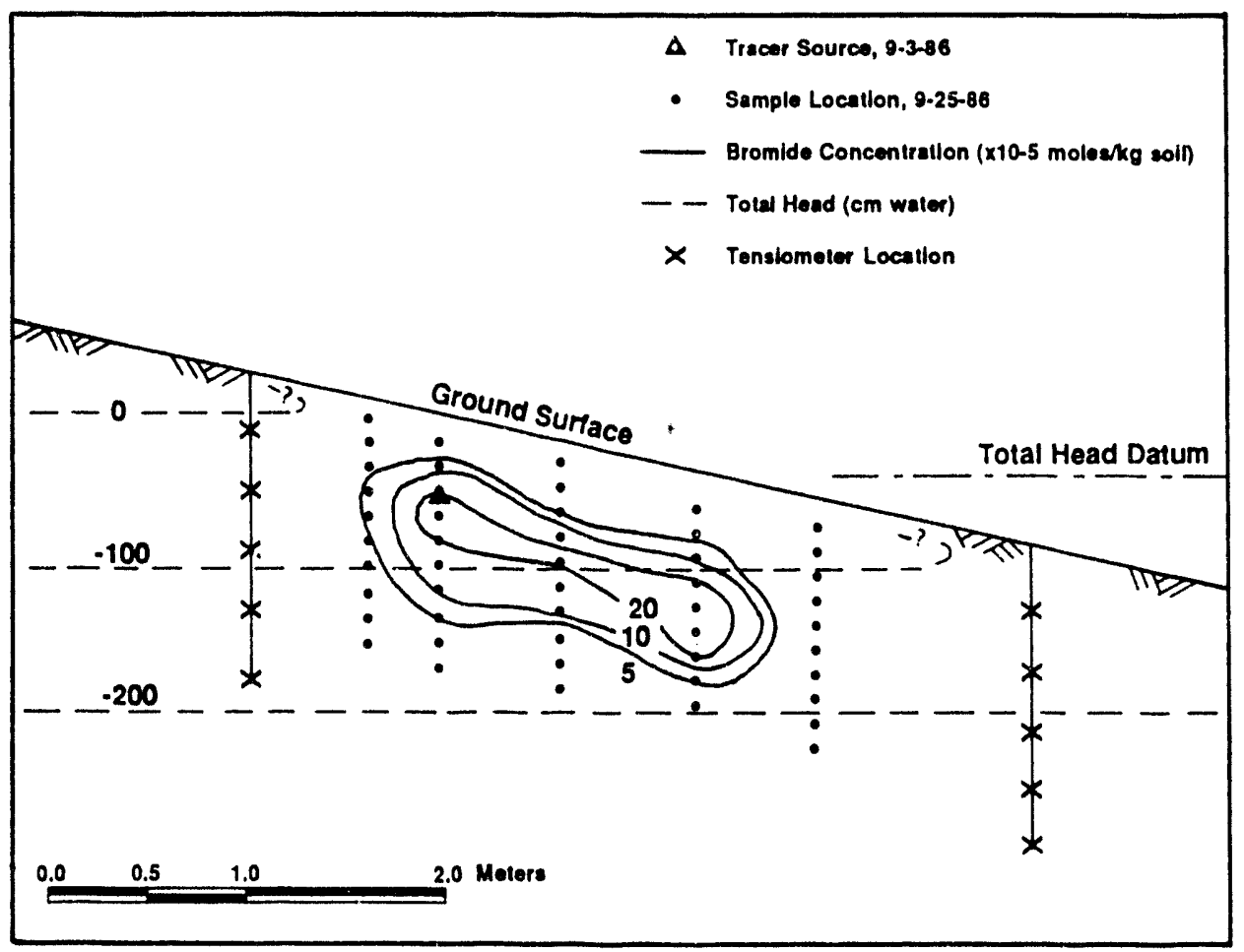

Figure 2-4. Contours of equal bromide concentration $\left(\times 10^{-5} \mathrm{~mol} / \mathrm{kg}\right.$ soil $)$ from hillslope tracer experiment on a sand dune (from McCord et al., 1991). 


\subsection{Applications of Stochastic Flow Theory}

Laboratory and field observations suggest that the effects of hydraulic heterogeneity, as reflected in the tension-dependent anisotropy of effective unsaturated hydraulic conductivity, are important; several field exampies are discussed by Yeh et al. (1985). A laboratory experiment with a layered sand (Stephens and Heermann, 1988) clearly shows that the lateral spreading of moisture is enhanced. A field experiment in a sand dune shows lateral migration of the tracer parallel to the hillslope even though the hydraulic gradient is vertical (see Figure 2-4). McCord et al. (1991) conclude that this observed behavior can be proprirly simulated only if a tension-dependent anisotropy of the effective conductivity is used.

Some simple analytical expressions can be used to illustrate the essential characteristics of the stochastic flow theory. For one-dimensional (in the mean) steady vertical infiltration into a heterogeneous soil with uniform mean tension, the results for the vertical $\left(\mathrm{K}_{11}\right)$ and horizontal $\left(\mathrm{K}_{22}\right)$ effective conductivities are:

$$
\begin{aligned}
& K_{11}=K_{g} \exp \left[-A^{*} H-\frac{\sigma_{f}^{2}+\sigma_{\alpha}^{2} H^{2}}{2\left(1+A \lambda_{1}\right)}\right] \\
& K_{22}=K_{g} \exp \left[-A^{*} H+\frac{\sigma_{f}^{2}+\sigma_{\alpha}^{2} H^{2}}{2\left(1+A \lambda_{1}\right)}\right] \\
& \frac{K_{22}}{K_{11}}=\exp \left[\frac{\sigma_{f}^{2}+\sigma_{\alpha}^{2} H^{2}}{1+A \lambda_{1}}\right] \\
& H=\text { mean tension } \\
& K_{g}=\exp \left\{E\left[\ln K_{s}\right]\right\}, \text { geometric mean } \\
& A=E[\alpha] \\
& \sigma_{f}^{2}=\operatorname{variance~of~} \ln K_{s} \\
& \sigma_{\alpha}^{2}=\text { variance of } \alpha \\
& \lambda_{1}=\text { vertical correlation scale } \\
& A^{*}=A\left[1-\frac{\sigma_{\alpha}^{2} \lambda_{1}}{A\left(1+\lambda_{1} A\right)}\right]
\end{aligned}
$$

These results are for the case with constant (independent of tension as in [2.1]) $\alpha$ which is not correlated with $\ln K_{s}$; also the soil is horizontally stratified (the horizontal correlation scale $\lambda_{2} \gg \lambda_{1}$ ). The corresponding expression for the tension variance is

$$
\sigma_{h}^{2}=\frac{\left(\sigma_{f}^{2}+\sigma_{\alpha}^{2} H^{2}\right) \lambda_{1}}{A\left(1+A \lambda_{1}\right)}
$$

Equations [2.3] through [2.6] demonstrate the strong dependence of the degree of anisotropy and the tension variance on mean tension particularly when the variance of $\alpha$ is large. These expressions can be used to make simple screening calculations of the 
large-scale unsaturated flow conditions under natural infiltration conditions. Ababo'! at a'. (1988) compared the vertical effective conductivity and the head variance predicted from these expressions with the results of detailed numerical simulations of steady infiltration into a three-dimensionally heterogeneous soil, and found generally favorable agreement (see their Section 7.4).

The use of the stochastically-derived effective conductivities and head variance has been demonstrated by Bensabui et al. (1992) in preliminary numerical simulations of the first experiment at the Las Cruces trench site (Hills and Wierenga, 1991). This modeling used the unsaturated flow code developed by Bouloutas (1989) which was modified to incorporate the effective conductivity results from the enhanced stochastic theory (Polmann, 1990). The variance of water content was also evaluated from the theory. The simulation of a four year experiment were carried out on a DEC 3100 workstation in a few hours. The results of the simulations were in general agreement with the field observations in terms of the overall pattern of moisture spreading as well as the degree of variability in moisture content. Deterministic (homogeneous) simulations clearly were not able to represent the extensive horizontal spreading seen in this long-term experiment. The input data on the variability of the unsaturated conductivity were synthesized from the Maddock soil and the saturated conductivity at the site because unsaturated conductivity data were not available for the Las Cruces site.

Numerical simulations of a two-dimensional stochastic mean flow configuration similar to the Las Cruces experiment have been compared with detailed simulations using a synthetically generated three-dimensional heterogeneous soil (Polmann et al., 1991). The synthesized medium was used so as to permit definitive comparisons using known soil variability parameters. Figure 2-5 summarizes the flow conditions and boundary conditions used in the simulations. Water was applied uniformly over the wetted strip for 10 days followed by a period of redistribution with no surface flux. Results of the simulations at 15 days are illustrated in Figure 2-6 which shows, in the top three panels, the detailed tension distribution for three different vertical sections across the infiltration strip; the bottom panel shows the corresponding result of the stochastic mean flow simulation. The upper panels clearly show the influence of the soil heterogeneity in creating a very irregular distribution of tension. Such irregular variation will certainly be encountered in the field, but the details will be unpredictable and usually of minimal applied interest. In contrast the lower panel reflects the smoothed or aggregated behavior of the moisture plume as predicted by the mean flow model using the using the effective parameters derived from stochastic theory. The stochastic result seems to capture the overall behavior, but the detailed results are so irregular that it is difficult to judge visually whether the stochastic results are adequate. The comparison can be viewed more quantitatively by including the predicted tension variance as illustrated in Figure 2-7; this shows the mean simulation $\pm 2 \sigma_{h}$ along with the detailed simulations for the three sections. It is seen that the mean simulation does seem to represent the central tendency of the detailed simulations and that most of the variation falls within the $\pm 2 \sigma_{h}$ interval around the mean. This notion of using the variance of the predicted variable as a measure of acceptable error is explored more systematically in Chapter 4 in addressing the issue of model validation. 



Figure 2-5. Detailed and mean simulation domains (from Polmann et al., 1991). 


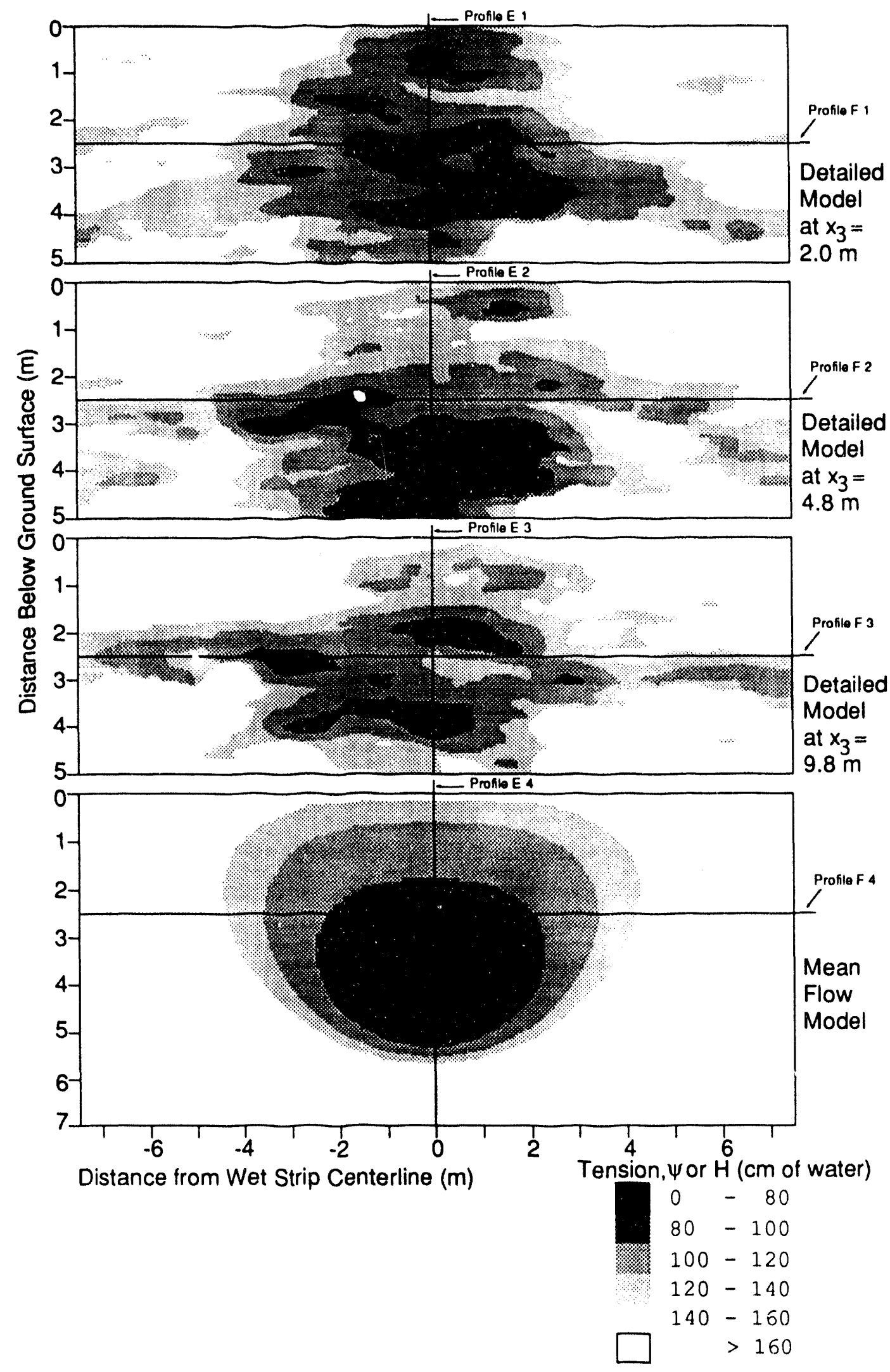

Figure 2-6. Tension distributions for the detailed and mean simulations at 15 days (from Polmann et al., 1991). 
(a.)

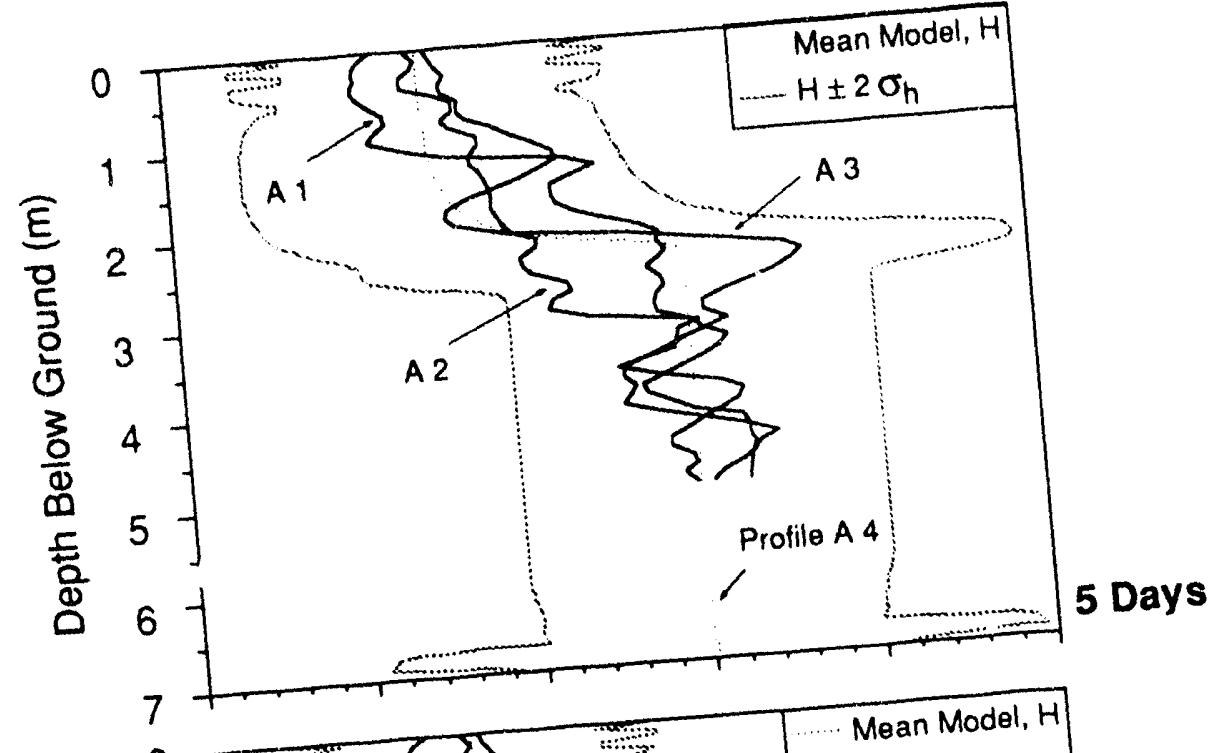

(b.)

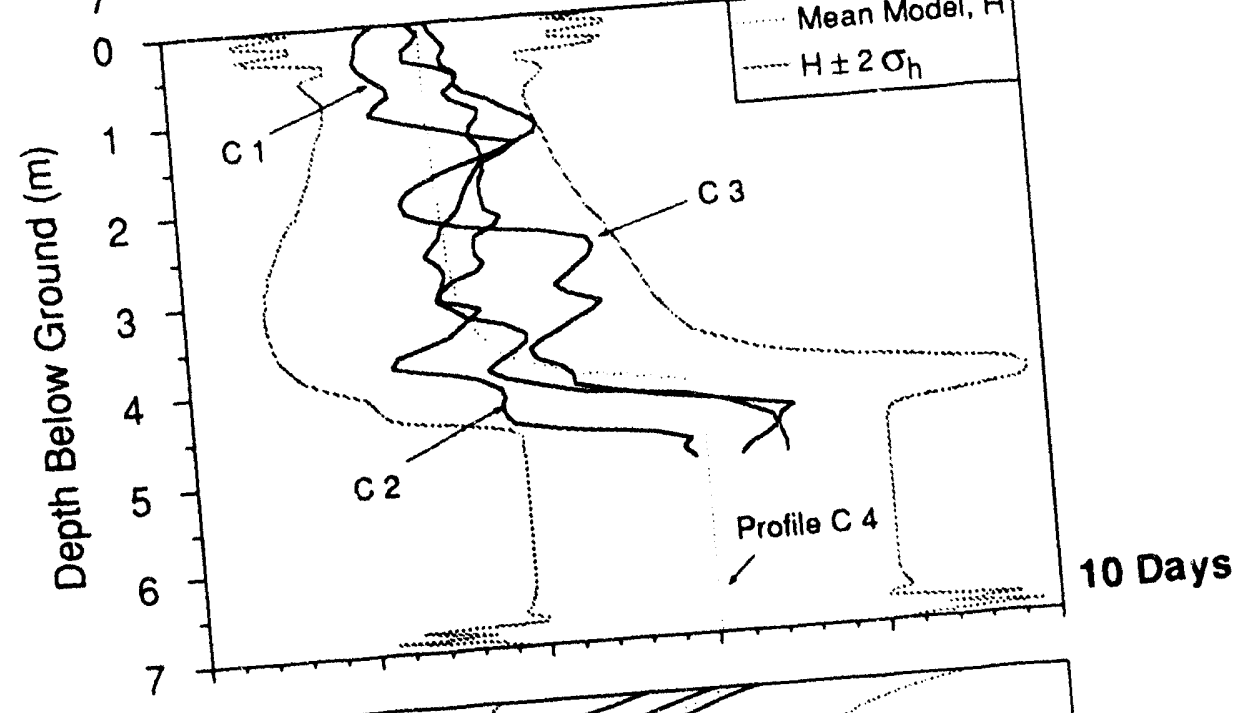

(c.)

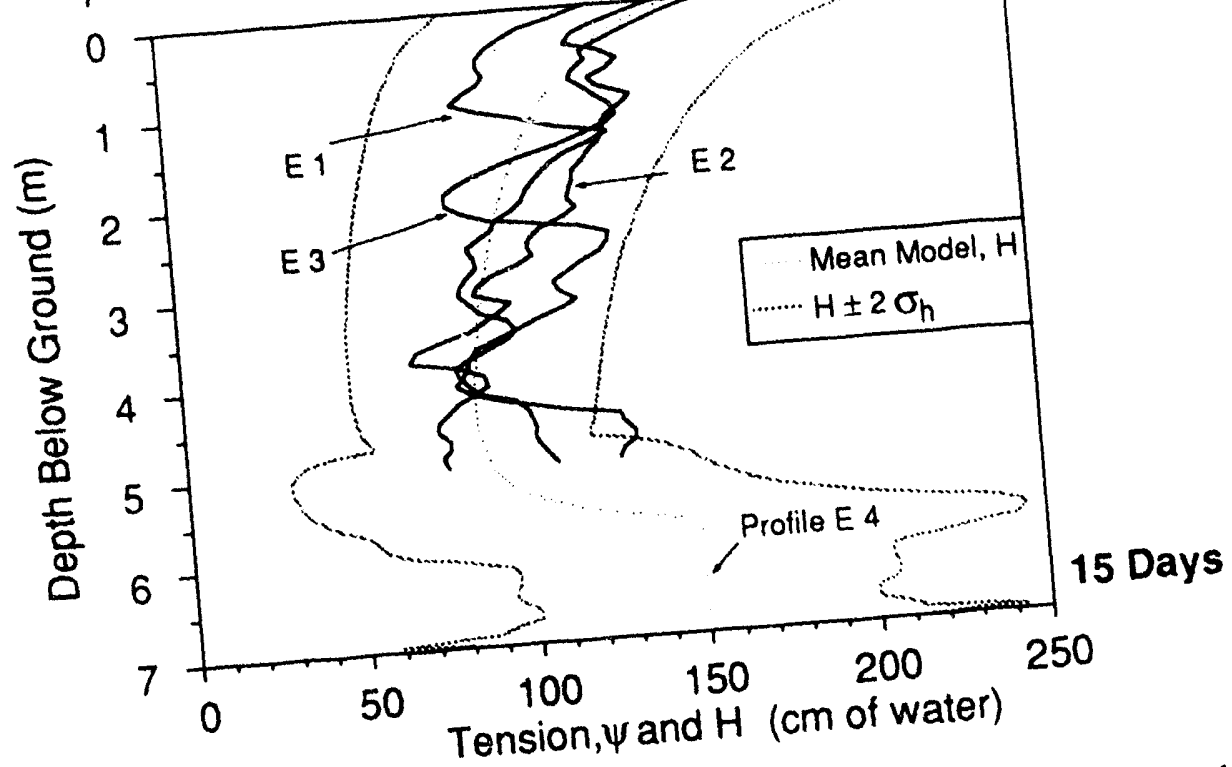

Figure 2-7. Vertical tension profiles under wetted strip centerline for the detailed and mean simulations (from Polmann et al., 1991) 
Overall, the experience in applying stochastic mean flow models demonstrates that with the enhanced stochastic theory and improved numerical simulation techniques it is now feasible to simulate realistic two-dimensional field conditions on scales of tens of meters and extending decades. The stochastic approach, which incorporates the effects of complex small-scale variability through effective parameters, is attractive because it provides a simplified aggregated representation which derives explicitly from small-scale measurements of hydraulic properties and their variability.

\subsection{Stochastic Unsaturated Contaminant Transport}

A stochastic treatment of transport of a nonreactive solute under unsaturated flow conditions has to be developed as part of the project research by using the spatially variable velocity field predicted from the flow theory in the governing transport equation (Mantoglou and Gelhar, 1985; Polmann, 1990). This theoretical approach is well established in the case of saturated flow (Gelhar and Axness, 1983; Dagan, 1984) and has been independently confirmed through carefully designed field experiments which show that the macrodispersivity can indeed be predicted from measurements of the spatial variability of saturated hydraulic conductivity (Sudicky, 1986; Garabedian et al., 1991). In the unsaturated case the theory shows that the macrodispersivity is influenced primarily by the variations in the unsaturated hydraulic conductivity with only a minor effect of the variation in moisture content (Polmann, 1990).

The unsaturated theory indicates that the longitudinal macrodispersivity increases modestly with increasing tension or the soil becomes drier (see Figure 2-8). In the unsaturated case the existing field experiments are not adequate to test the transport theory but some recent two-dimensional numerical simulations of solute transport in heterogeneous unsaturated soils indicate that the mixing processes can be described in a fashion similar to that for saturated flow (Russo, 1991).

Field observations of dispersion in unsaturated flow, as summarized in Figure 2-9, show that field-scale dispersivities are significantly larger than laboratory values and are of the same order of magnitude as predicted by the stochastic theory (Figure 2-8). There is no scientific consensus about how to characterize dispersion in unsaturated flow. This is evident from the fact that laboratory column tests continue to be advocated as a method of characterizing waste disposal sites (Wierenga et al., 1991). Clearly there is a need for carefully designed field tracer experiments extending over tens of meters with measurements of spatially variability of hydraulic parameters in order to evaluate the predictive capabilities of the unsaturater stochastic transport theory.

Transport of reactive solutes can be significantly influenced by chemical heterogeneity of the soil. This process has been analyzed for the case of saturated flow with a solute undergoing linear equilibrium sorption (Garabedian et al., 1988); it was found that the effective retardation factor in this case is the arithmetic mean and that the longitudinal macrodispersivity of a soring solute is increased relative to that for a nonreactive solute when there is a negative correlation between the sorption characteristic $K d$ and the log- 
conductivity, and is always increased by a $K d$ variation which is not correlated with the conductivity. The tracer test on Cape Cod (Garabedian et al., 1988) shows a significant enhancement (roughly a factor of 10 ) of the longitudinal macrodispersivity for a reactive tracer (lithium) relative to that for the nonreactive tracer (bromide) but complications in the reactive process preclude a definitive resolution of the role of chemical heterogeneity in this case. Robin et al. (1991) report on extensive muasurements of the variation of sorption properties for the Borden aquifer; those measurements show substantial variations in $K d$ but only a weak correlation with log-conductivity. It is important to consider the likely enhancement of the longitudinal dispersivity of heterogeneously sorbed species because it is now common practice to assume (without any real justification) that the same dispersivity applies to all transported species. The effects of spatially variable sorption have not been explored explicitly for the case of unsaturated flow, but, aside for some additional relatively minor effects due to variations in moisture, a similar enhancement of mixing could be anticipated.

Project research also addressed the influence of soil heterogeneity on diffusive vapor transport. Using a stochastic treatment, it has been found that the effective diffusion coefficient becomes a tensorial property with only a modest degree of anisotropy for perfectly dry soils (at most a factor of 2) but as much as an order of magnitude decrease in the effective diffusivity in the direction perpendicular to bedding in the more realistic case of moist soils (Ding, 1992). The calculations, which are based on the Polmann (1990) theory for the variability of moisture content applied to the Maddock soil, show that the horizontal (parallel to bedding) effective molecular diffusivity is 10 times the vertical diffusivity for a water saturation of 0.6 (see Figure 3-4, Ding, 1992). The implication is that transport of gaseous contaminants in the horizontal direction would be enhanced and vertical transport would be restricted. 




Figure 2-8. Longitudinal macrodispersivity versus mean tension for Maddock soil under steady-state flow with uniform mean tension (from Polmann, 1990).

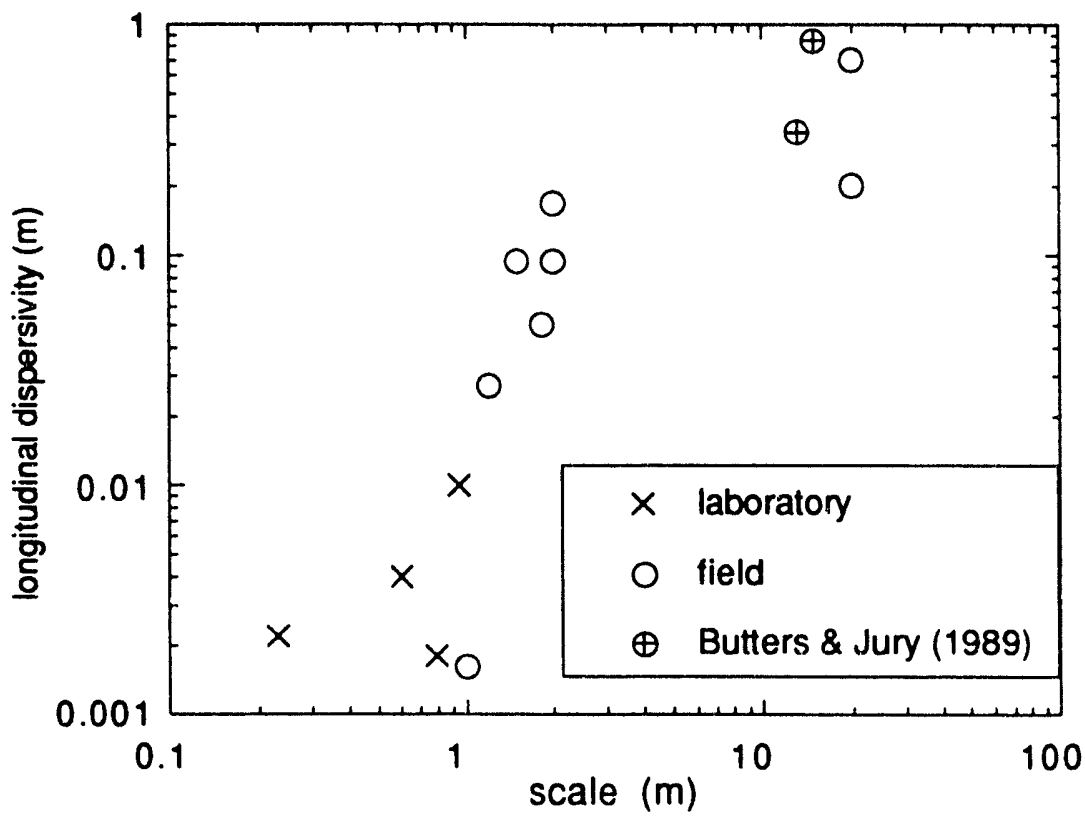

Figure 2-9. Longitudinal dispersivities for unsaturated soils as related to displacement scale (from Gelhar, 1992). 


\section{CHAPTER 3}

\section{NUMERICAL MODELING AND VAPOR TRANSPORT}

\subsection{Governing Equations}

To describe mathematically the movement of fluids and contaminants in the unsaturated zone, balance of mass equations are written for the quantities of interest. For example, to describe the fluid motion, the appropriate mass balance equations have the following form:

$$
\frac{\partial\left(n \rho^{\alpha} S^{\alpha}\right)}{\partial t}+\nabla \cdot\left(\rho^{\alpha} \mathbf{q}^{\alpha}\right)=F^{\alpha}, \quad \alpha=a, w
$$

In Equation [3.1], $n$ is porosity, $\rho^{\alpha}$ is density of fluid $\alpha, S^{\alpha}$ is saturation of phase $\alpha, \mathbf{q}^{\alpha}$ is the Darcy flux vector for phase $\alpha, F^{\alpha}$ represents sources or sinks of mass to or from phase $a$, and $a$ represents the two fluid phases present, air $(a)$ and water $(w)$. This equation is written for each of the fluids. In addition to these balance equations, materialspecific relationships must be specified. The usual expressions are the multi-phase version of Darcy's law, and relationships between relative permeability and fluid saturations and between saturations and the difference between the pressures of the two fluids (the so-called "capillary pressure"). Mathematically these take the following forms:

$$
\begin{aligned}
& \mathbf{q}^{\alpha}=-\frac{\mathbf{k} k_{r \alpha}}{\mu^{\alpha}} \cdot\left(\nabla p^{\alpha}-\rho^{\alpha} \mathbf{g}\right) \\
& S^{\alpha}=S^{\alpha}\left(p^{\alpha}-p^{w}\right) \\
& k_{r \alpha}=k_{r \alpha}\left(S^{\alpha}\right)
\end{aligned}
$$

Here, $\mathbf{k}$ represents intrinsic permeability, $k_{r \alpha}$ is the relative permeability of the medium to phase $\alpha, \mu^{\alpha}$ is the viscosity of fluid $\alpha, p^{\alpha}$ is the pressure of fluid $\alpha$, and $\mathbf{g}$ is the gravitational acceleration vector.

Equations [3.1] and [3.2] represent a set of twelve equations for the twelve unknowns $\left\{S^{\alpha}, p^{\alpha}, \mathbf{q}^{\alpha}, k_{r \alpha}\right\}$. The complex, nonlinear nature of this equation set makes solution very difficult. Therefore simplifying assumptions and numerical solution techniques are usually used.

One of the standard simplifying assumptions is based on the following observation: Because the viscosity of air is much less than that of water, the air can move much more easily (has a much higher "mobility") than the water. Therefore movement of significant quantities of air requires only small pressure gradients, which often means that the air pressure does not deviate significantly from atmospheric pressure throughout the soil. Thus a standard assumption, called the Richards assumption, is that the air pressure remains essentially constant, equal to atmospheric pressure (zero gage pressure). Then 
$p^{a}-p^{w}=-p^{w}, S^{w}=S^{w}\left(p^{w}\right)$, and all equations related to the air phase are eliminated. The equation set that results is usually referred to as Richards equation.

As discussed below (Section 3.3) and in more detail in Celia and Binning (1992a), determination of whether or not Richards equation is an adequate representation of an unsaturated system depends largely on the objectives of the analysis. If water movement and associated transport of non-volatile contaminants are the issues, then Richards equation is usually adequate. If, however, volatile contaminants are involved, such that movement in the air phase is likely, then solution of the air-phase equations is probably warranted.

\subsection{Numerical Methods for Richards Equation}

As detailed in Celia et al. (1990a), numerical solution of Richards equation depends on the form of the governing partial differential equation used in the analysis, and it depends critically on how the time derivative in that equation is treated. A summary of these concepts is provided in this section.

Richards equation is usually written in one of three common forms:

(i) $h$-based form

$$
C(h) \frac{\partial h}{\partial t}-\nabla \bullet(\mathbf{K} \bullet \nabla h+\nabla z)=0
$$

(ii) $\theta$-based form

$$
\frac{\partial \theta}{\partial t}-\nabla \cdot(\mathbf{D} \cdot \nabla \theta+\nabla z)=0
$$

(iii) Mixed form

$$
\frac{\partial \theta}{\partial t}-\nabla \bullet(\mathbf{K} \bullet \nabla h+\nabla z)=0
$$

In these equations, fluid and matrix compressibilities are ignored for convenience of presentation (they are always included in the computer codes), $C(h)$ is the specific moisture capacity function, $\mathbf{D}$ is the soil diffusivity tensor, and $\mathbf{K}$ is the unsaturated hydraulic conductivity. Historically, the $h$-based form has been used in hydrology, the $\theta-$ based form has been used in soil science, and the mixed form has been used (in a somewhat different zontext) in the petroleum industry.

In our research, we have concluded that the mixed form of the Richards equation, coupled with certain linearization techniques, is preferable to the more standard $h$-based formulations. The reasons for this are as follows: (i) while $h$-based formulations apply easily to both saturated and unsaturated conditions, the numerical approximations are non-mass-conservative unless special corrections are introduced; (ii) $\theta$-based formulations typically conserve mass, but they become ill-posed in the saturated zone; 
(iii) approximations based on the mixed form of the equation lead to mass-conservative solutions that apply easily to both saturated and unsaturated conditions. Mass balance results for a simple one-dimensional test problem demonstrate the importance of mass conservation. This problem is one of infiltration into an initially dry column of sand, with parameters being initial estimates of those that apply to the Las Cruces field site (Bouloutas, 1989; Polmann, 1990; Wierenga et al., 1991). Figure 3-1 shows the sensitivity of solutions to time step size: $h$-based simulators have pronounced timetruncation errors, leading to a loss of mass conservation, while the mixed formulation is essentially insensitive to time step for the same parameters. Figure 3-2 summarizes mass balance results, demonstrating the mass-balance problems of the $h$-based formulation.

In Celia et al. (1990a), we also point out that standard finite element solutions can perform poorly for problems involving infiltration into initially dry soils. Mathematically, this is because the time matrix is not diagonal, which in turn means that the numerical solution is not required to respect a maximum principle (which the governing partial differential equation does respect). Because the solution is not required to respect a maximum principle, non-physical oscillations can occur, typically ahead of a steep infiltrating front. The solution to this problem is diagonalization of the time matrix ("mass lumping"). When diagonalization is applied, linear analysis indicates that numerical solutions obey a maximum principle (Bouloutas, 1989) such that physically smooth infiltration fronts remain numerically smooth.

Numerical solutions that respect both mass conservation and a maximum principle are not guaranteed to be "good" approximations. However adherence to these two principles usually indicates a reasonable numerical solution. Conversely, solutions that exhibit up to $50 \%$ mass balance errors, such as those examined in Celia et al. (1990a), are clearly not acceptable.

Note that the basic principles discussed above, and presented in more detail in Celia et al. (1990a) and Celia and Binning (1992a), serve to explain why certain numerical approaches should be preferred. The importance of mass conservation and a respect for a maximum principle are clearly demonstrated. While more sophisticated methods, such as mixed finite element methods and variants thereof (Ewing et al., 1984; Kirkland et al., 1992) might produce improved efficiencies, the fundamental principles discussed in Celia et al. (1990a) continue to apply.

A final consideration in the solution of Richards equation is the matrix solution step. We have examined a number of alternatives, and concluded that a preconditioned conjugate gradient algorithm, with incomplete Cholesky preconditioning, is a very good choice for the Richards equation (Bouloutas, 1989; Bouloutas and Celia, 1992). Because unsaturated flow problems tend to require relatively small time steps, even diagonal preconditioning performs quite well. This is an important observation for parallelization of these algorithms, because diagonal scaling is an especially simple preconditioner to implement on parallel machines (Celia et al., 1992). 
FINITE DIFFERENCE SOLUTIONS, $\Delta Z=2.5 \mathrm{CM}$



FINITE DIFFERENCE SOLUTIONS (MODIFIED PICARD), $\triangle Z=2.5 \mathrm{CM}$

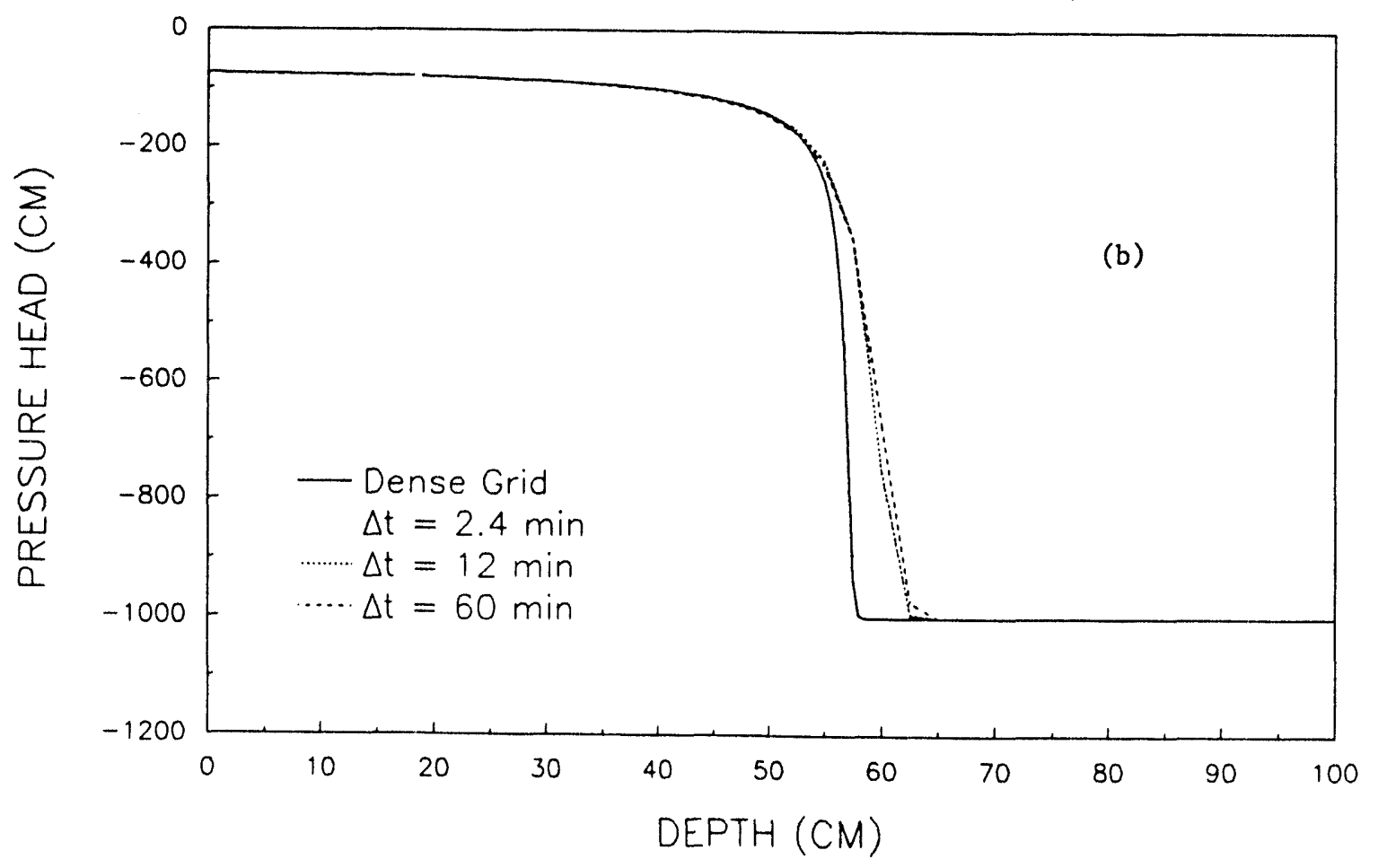

Figure 3-1. Numerical solutions using (a) $h$-based simulator and (b) mixed formulation (from Celia et al., 1990a). 


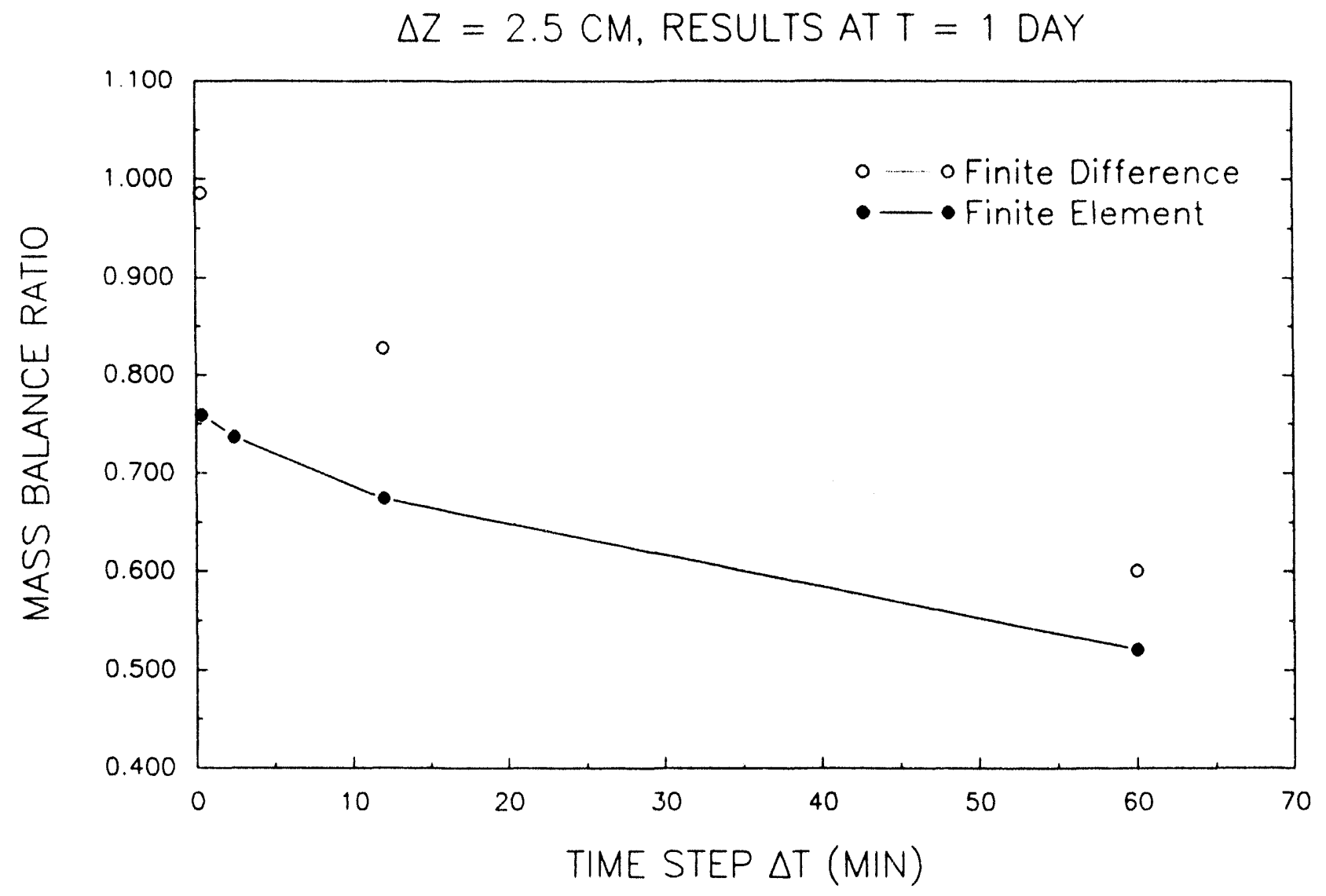

Figure 3-2. Mass balance result for finite element and finite difference solutions to the $h$ based equation. Mixed formulation solutions produre mass balance ratios equal to one for all time step sizes (from Celia et al, 1990a). 


\subsection{Inclusion of Air-Phase Dynamics}

When solving the equations that include air-phase dynamics, Equation [3.1] must be written for both the water and air phases. Now the system involves a set of coupled, nonlinear partial differential equations, whose solution is more complicated than that for the Richards equation. However, because the equations are similar in form to Richards equation, the algorithms developed for Richards equation may be extended directly to the two-phase flow case. Therefore, the two-phase numerical algorithms we have developed use a lumped finite element approximation coupled with a modified Picard linearization of the mixed form of the equations. This again provides favorable behavior in terms of mass balance and a maximum principlc. Details of the algorithm may be found in Celia and Birning (1992a). Note that we are cuirently pursuing improved numerical algorithms for this set of equations, based on fractional flow concepts. A description of this may be found in Celia and Binning (1992b). The solutions presented below are all based on the algorithm of Celia and Binning (1992a).

One way that a two-phase (air-water) simulator may be used is to demonstrate when Richards approximation is reasonable, and what information is lost when Richards equation is used. For example. consider a soil column with an initial moisture distribution corresponding to gravity drainage, which is subjected to a constant rainfall rate at the top surface. If the rainfall rate is higher than the vertical saturated conductivity of the medium, then, after some time, ponding will occur at the surface. The bottom boundary is either sealed (no flow of either fluid) or open to flow of both fluids. This is shown schematically in Figure 3-3. By examining all combinations of boundary conditions, one can observe that the only time that Richards equation does a poor job of describing water movement is when ponding occurs and the bottom is sealed. For the other sases Richards equation is a good apnroximation. However, the most interesting information from these simulations is the response of the air phase. As an example, consider the case of relatively low rainfall rate (no ponding) and an open bottom boundary. In this case, the air pressure remains close to atmospheric (the maximum deviation is approximately $2 \mathrm{~cm}$ (water equivalent) pressure head. However, as shown in Figure 3-4, there is significant movement of air in the column. And, even though the bottom of the column is open to air flow, a significant amount of air leaves the column through the top boundary; in this case more than one-third of the air that leaves the column in the first 100 minutes of infiltration does so through the top. The air particle trajectories clearly show how the air velocity bifurcates at the infiltrating water front. This demonstrates that if volatile contaminants are of concern, whether or not Richards equation is a good approximation for the motion of water may be an irrelevant question.

Other related two-phase flow results are presented and discussed in Celia and Binning (1992a). In particular, mass balance results for each of the phases are presented, and comparisons to $h$-based simulations are made. Also, specific discussion and demonstrations of the air-phase pressure profiles are presented. Details of the numerical methods may also be found in that reference. 


\section{Boundary Conditions for flux infiltration into bounded column}

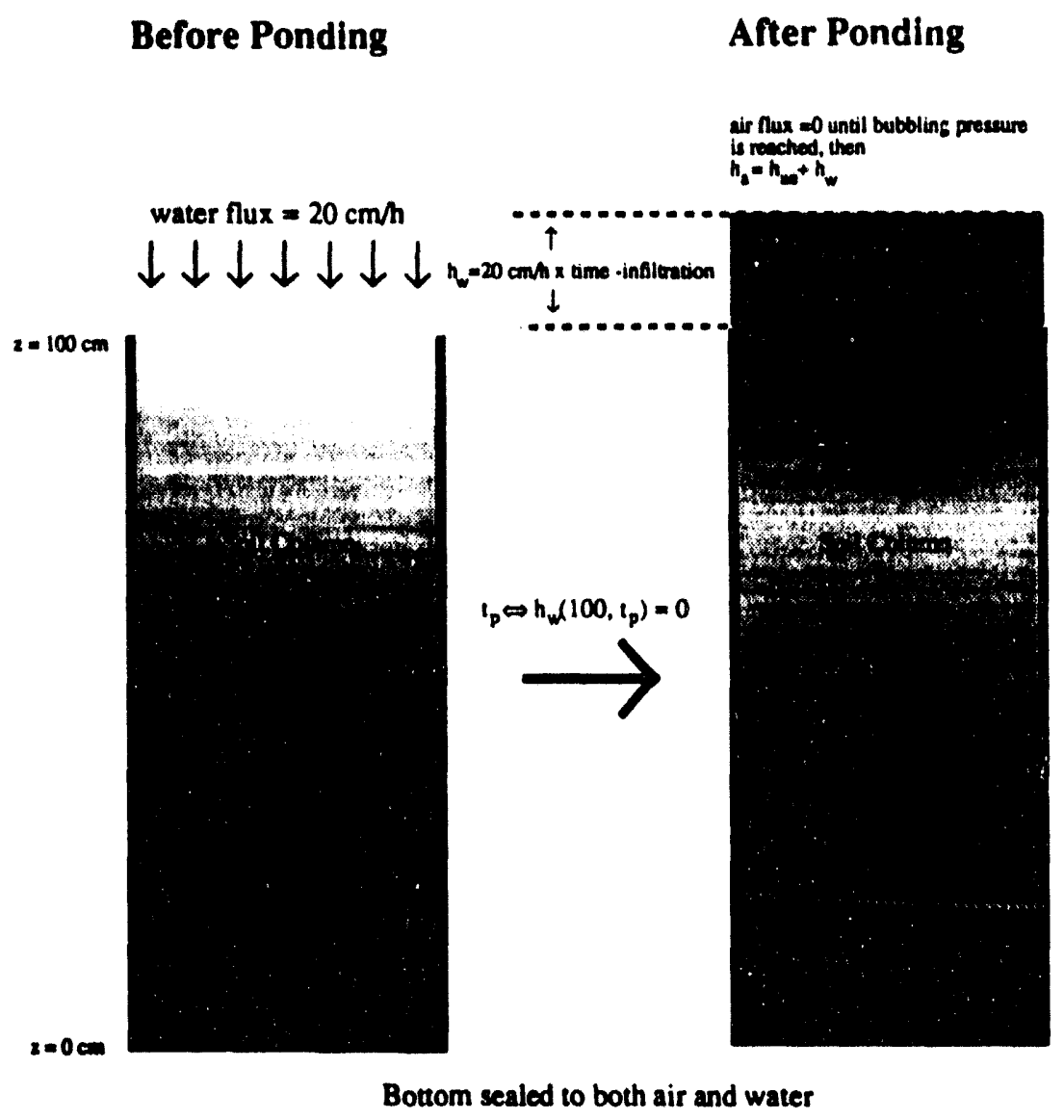

Figure 3-3. Experimental setup for air-water example showing boundary conditions, including possible ponding. Darker shading indicates higher water content (from Celia and Binning, 1992a) 


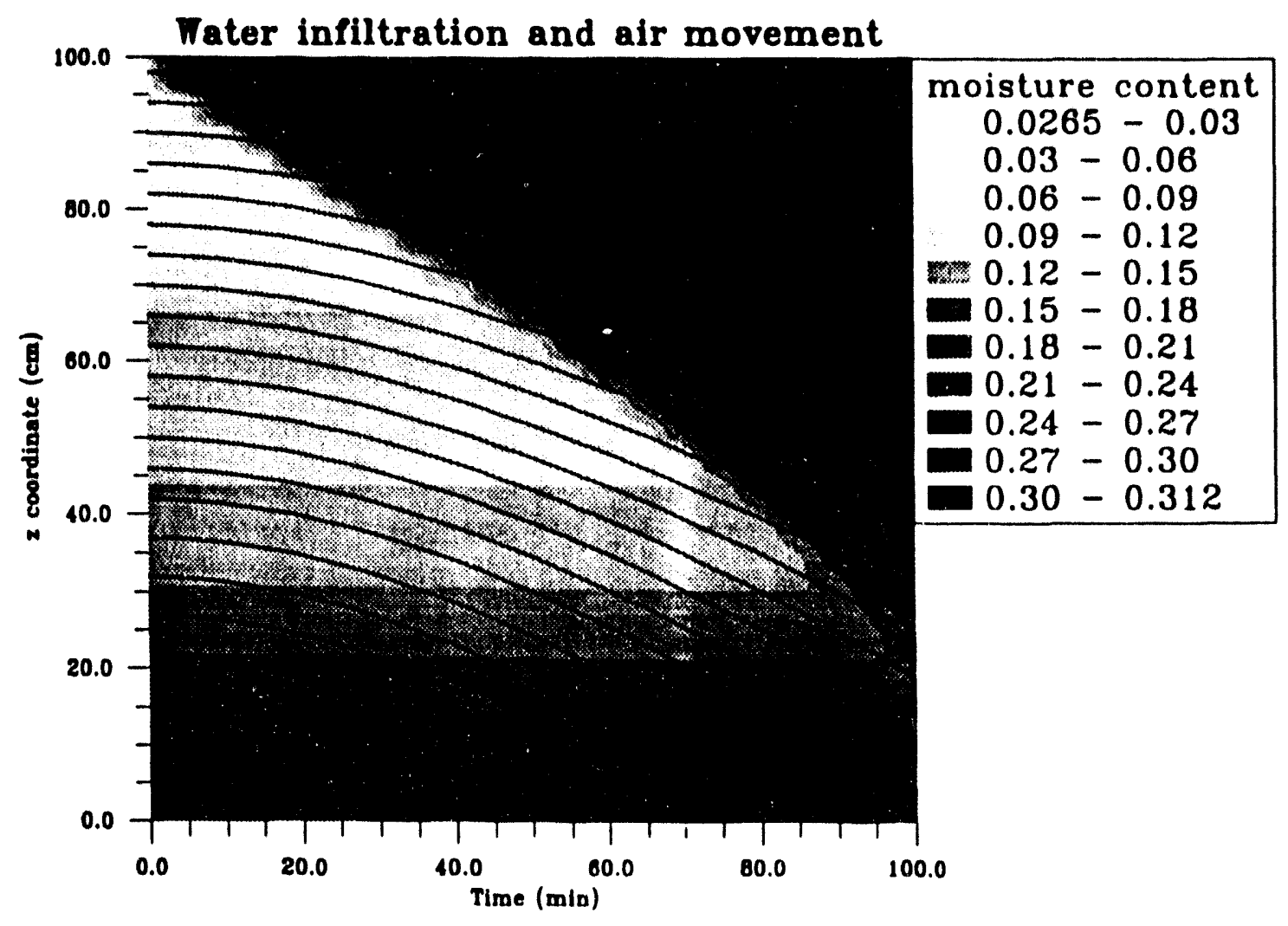

Figure 3-4. Time evolution of infiltrating water front (as shown in grey scale) and air movement (shown as particle trajectories) for column with open bottom and no ponding at the surface (from Celia and Binning, 1992a). 


\subsection{Modeling Contaminant Transport}

When modeling transport of contaminants, additional equations must be written and solved. These equations derive from conservation of mass and analogs of Fick's law of diffusion. The standard form is:

$$
\frac{\partial\left(n S^{\alpha} c_{i}^{\alpha}\right)}{\partial t}+\nabla \bullet\left(\mathbf{q}^{\alpha} c_{i}^{\alpha}\right)+\nabla \bullet \mathbf{J}_{i}^{\alpha}=F_{i}^{\alpha}, \quad \alpha=a, w
$$

where $c_{i}^{\alpha}$ is a measure of concentration of substance $i$ dissolved in phase $\alpha, F_{i}^{\alpha}$ is the net source of substance $i$ from outside of phase $\alpha$, including all other phases, and $\mathbf{J}_{i}^{\alpha}$ is the non-advective flux of substance $i$ in phase $\alpha$, assumed to be expressible in Fickian form as:

$$
\mathbf{J}_{i}^{\alpha}=-n S^{\alpha} \mathbf{D}_{i}^{\alpha} \bullet \nabla c_{i}^{\alpha}
$$

with $\mathbf{D}_{i}^{\alpha}$ being a diffusion/dispersion tensor.

Three different types of contaminant transport scenarios have been considered: (i) aqueous-phase contamination with no volatilization to the air; (ii) aqueous- and air-phase contamination with equilibrium partitioning between phases; and (iii) aqueous- and airphase contamination with nonequilibrium (kinetic) partitioning between phases. These different cases lead to different expressions for the right-hand side term, $F_{i}^{\alpha}$, in Equation [3.4]. These cases are treated separately below.

\section{Case 1 - No partitioning}

In this case, there is no need to write the air-phase transport equation, because by assumption no contaminant exists in the air phase. This also means that the air motion equation does not need to be solved, presuming Richards assumption is applicable. The transport equation has, to this point, been solved using standard finite element and finite difference methods. Improved computational methods, such as the ELLAM algorithms of Celia et al. (1990b), would make the calculations more efficient, but the general conclusions about flow and transport are unchanged.

As an example, consider the column experiments used previously when looking at water and air flow (Figures 3-3 and 3-4). Specifically, consider the case of infiltration without ponding (surface flux rate of $8.3 \mathrm{~cm} / \mathrm{hr}$ ). Both open and sealed bottom boundaries have been considered. Let a source of contamination be buried at some distance below the surface, as illustrated in Figure 3-5. For this example, with no partitioning to the air phase, the concentration profile evolves with time as shown in Figure 3-6. Notice that the area under the plots of concentration as a function of depth, for different times, is not constant. This does not indicate a loss of mass, rather it simply reflects the changing moisture content profile. 


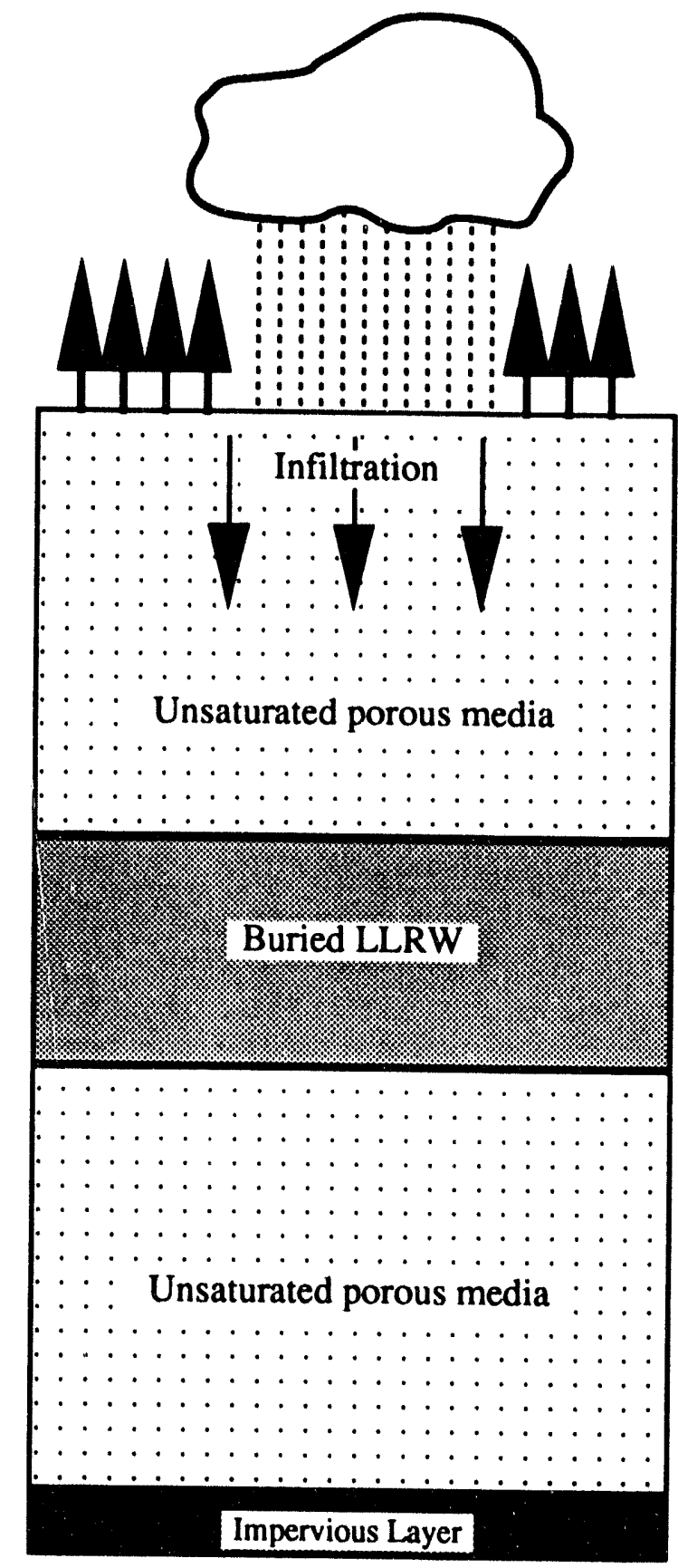

Figure 3-5. Schematic for contaminant transport simulations (from Sweed et al., 1992). 
Case 2 - Equilibrium partitioning

For this case, the concentrations in the water and air phases are assumed to be in equilibrium, related by Henry's Law. The general expression is

$$
c_{i}^{\alpha}=H_{i} c_{i}^{w}
$$

where $H_{i}$ is a modified Henry's law constant (see Sweed et al., 1992). When this is used, the air and water transport equations may be combined into one equation, as detailed in Sweed et al. (1992). For this case, we have considered a number of chemical compounds, including TCE (for which significant experimental data has been measured at Princeton) and triated methane (which might be a typical contaminant at a LLW burial site). Detailed results are presented in Sweed et al. (1992). As an example, consider TCE. Results analogous to those of Figure 3-6 are shown in Figure 3-7. Notice that the profile is much more diffuse, due to the partitioning between phases and the difference in phase velocities.

For equilibrium partitioning, the concentration profiles are highly dependent on the Henry's constant. For example, if triated methane is taken as the contaminant, then a significant quantity reaches the land surface quite quickly. This is because large values of $H$ weight the net flux vector toward the air-phase velocity. See Sweed et al. (1992) for details.

Case 3 - Non-equilibrium partitioning

The non-equilibrium partitioning uses the following kinetic exchange expression:

$$
F_{i}^{w}=-F_{i}^{\alpha}=K \theta_{\alpha}\left(c_{i}^{\alpha}-H_{i} c_{i}^{w}\right)
$$

where $\theta_{a}$ is the fluid content of air (equal to porosity minus moisture content). This expression introduces an additional parameter, which is the rate coefficient $K$. For demonstration purposes, we have run a set of simulations using rate coefficients measured at Princeton for TCE. For details, see Sweed et al. (1992) and Cho (1992). Figures 3-8 and 3-9 show water and air phase concentration profiles for the case of $K=0.07 \mathrm{~h}^{-1}$ which is at the lower end of the range of values for TCE partitioning in airwater systems. Now the profiles in the water phase are similar to those for Case 1, the nopartitioning case. However, the air-phase concentrations are significantly different than those in the water, owing to the different phase velocities in the air and water and the lack of equilibrium conditions.

These three simple examples demonstrate the importance of understanding the partitioning behavior of a specific waste components. They also demonstrates the potential importance of air-phase velocities. Additional details may be found in Sweed et al. (1992). 


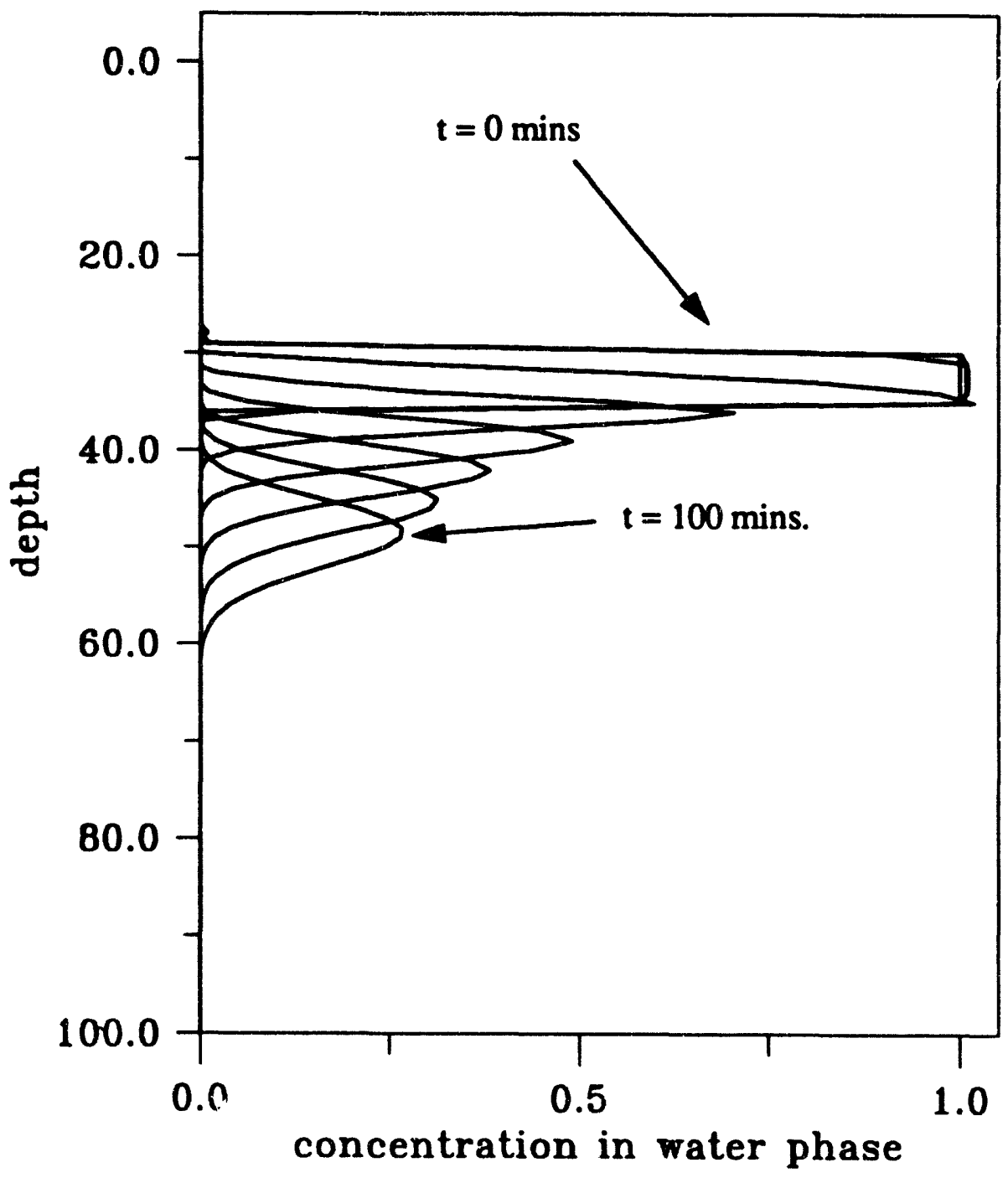

Figure 3-6. Contamination transport only in the water phase. Concentration profiles are shown at 10 minute intervals. 


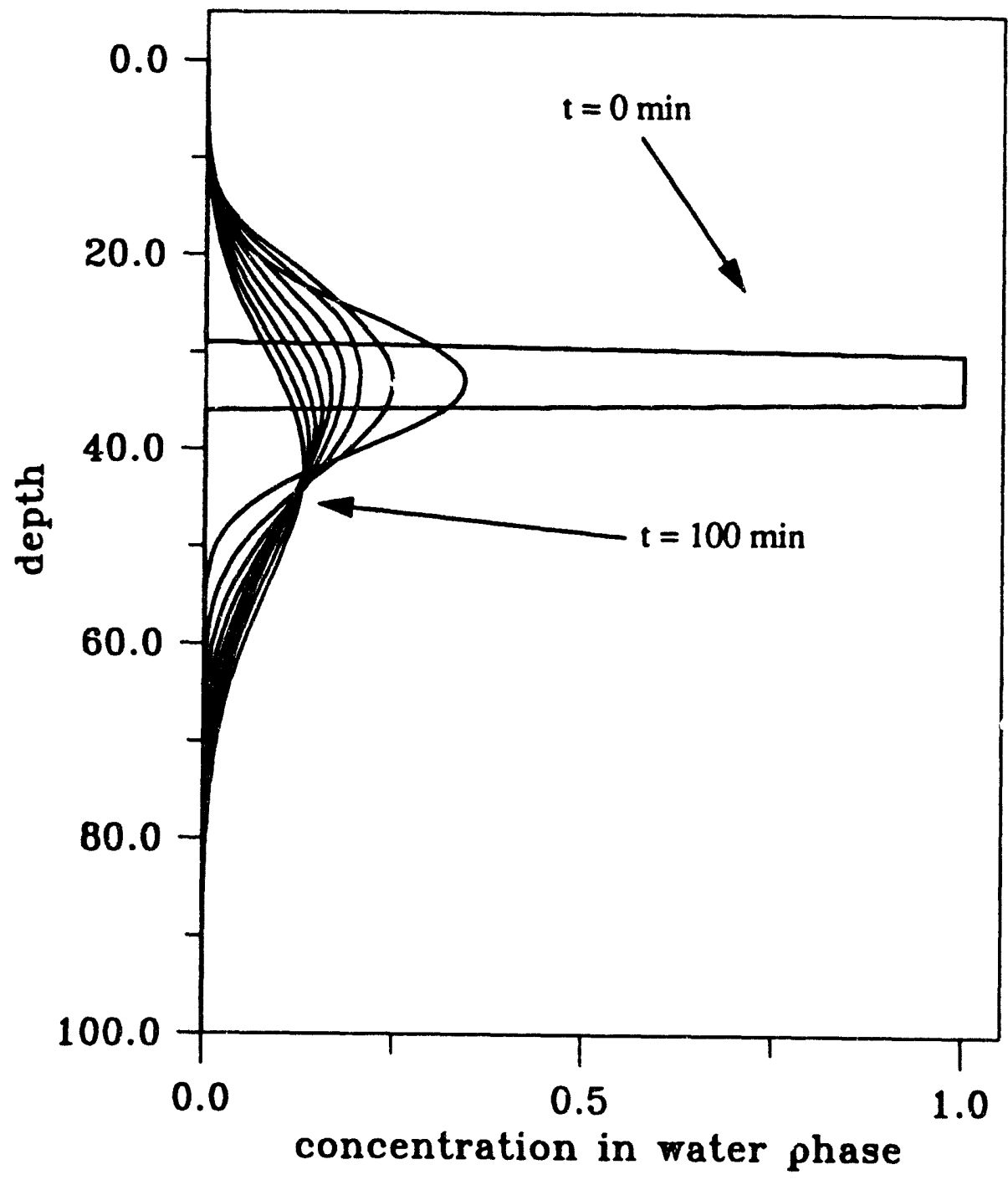

Figure 3-7. Contaminant plume evolution in time with equilibrium partitioning between the water and air phases. Concentration in the air phase differs from the water-phase concentration by Henry's constant. 


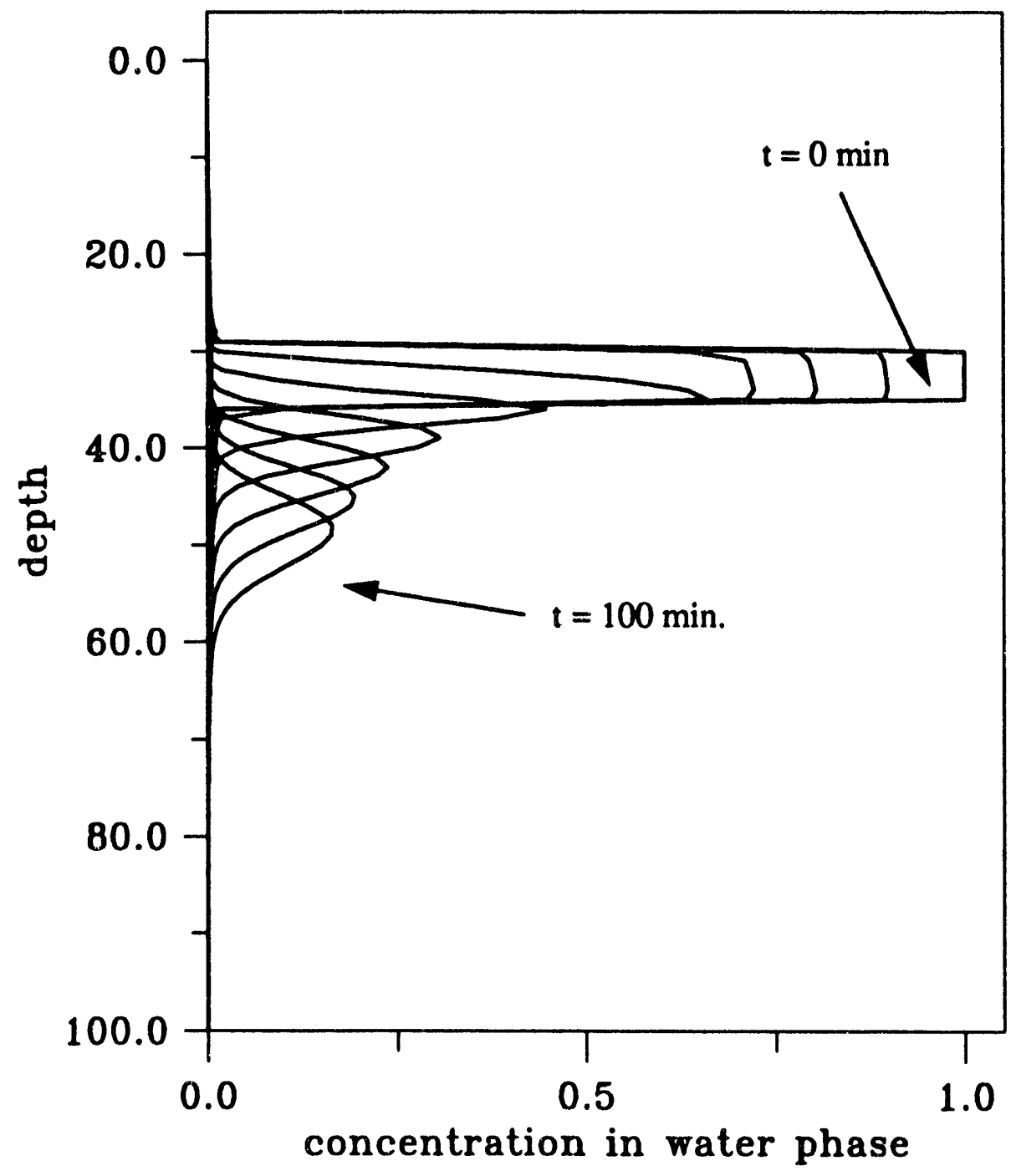

Figure 3-8. Concentration in the water phase with a kinetic mass transfer between the water and air phases. 


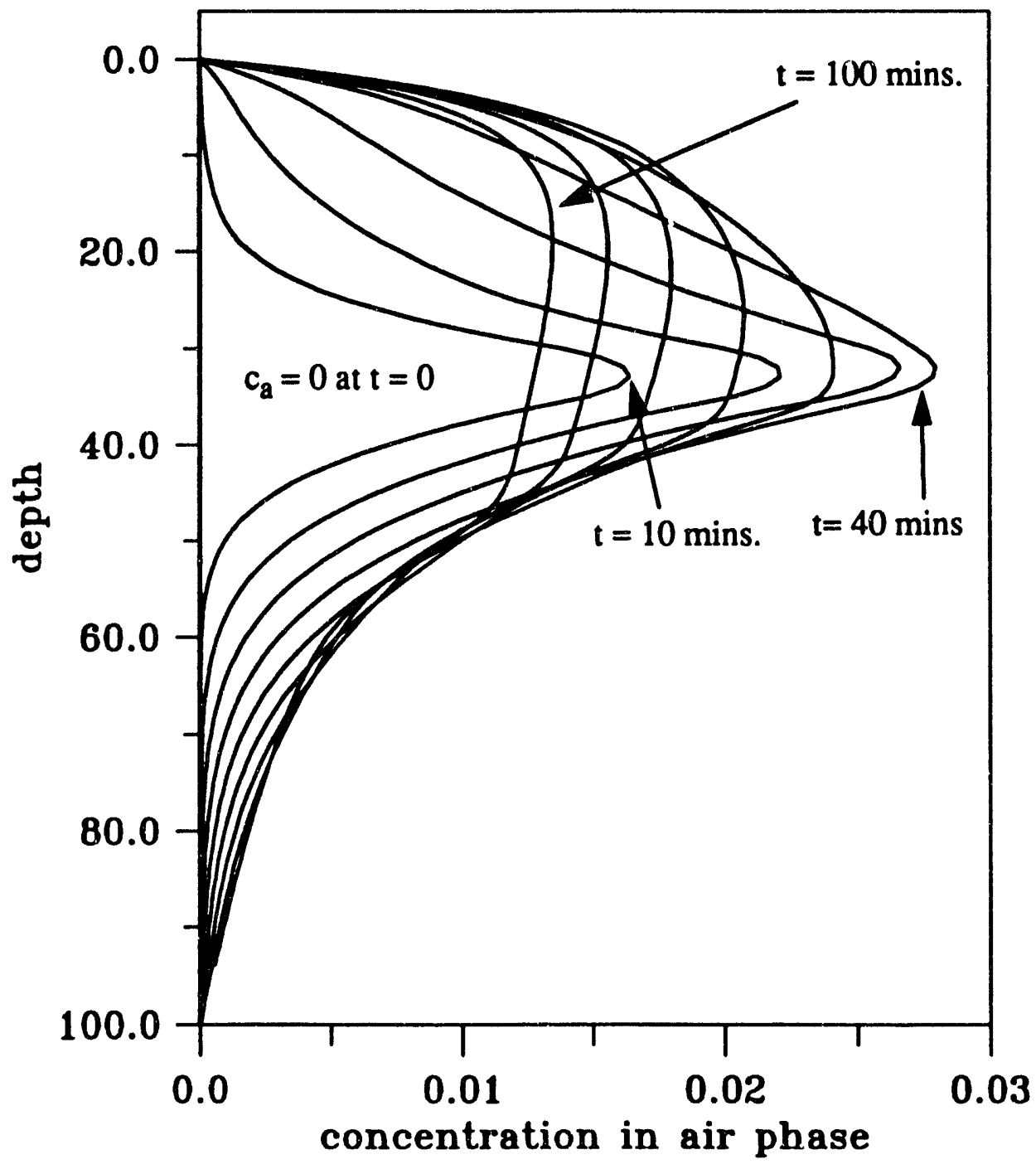

Figure 3-9. Concentration in the air phase for kinetic mass transfer with initial condition $\mathrm{ca}=0$ and $\mathrm{cw}=1$ between $30-35 \mathrm{~cm}$ depth. 


\subsection{Conclusion}

When solving unsaturated flow and transport equations, using either Richards equation or the more general two-phase equations, reliable numerical methods are prerequisite. Our research has shown why certain approximation methods perform better than others, based on concepts of mass conservation and control of non-physical oscillatory behavior. Detailed analysis of approximations for Richards equation has led to these conclusions. These detailed analyses also provide the basis for the design and implementation of numerical approximations for the more general two-phase flow case. Our method of choice uses the mixed form of the governing equations coupled with a modified Picard linearization procedure and a lumped finite element approximation in space.

When volatile contaminants are present in the unsaturated zone, vapor transport must be considered. In this case, both the movement of the air phase and the partitioning of contaminants between the water and air phases must be understood and properly incorporated into numerical models. While Richards equation may provide a good estimate of the water movement, it provides no information about the air phase motion. As such, two-phase simulations may be necessary to adequately analyze contaminant transport in the vapor phase. 


\section{CHAPTER 4}

\section{PERFORMANCE ASSESSMENT, SITE CHARACTERIZATION AND MODEL VALIDATION}

\subsection{Introduction}

The ultimate goal of most of the theoretical and modeling work described in this report is to provide tools for evaluating the performance of low-level radioactive waste (LLW) disposal facilities. Ideally, performance evaluations should establish beyond a reasonable doubt whether or not a given site will meet stated safety requirements. In reality, we must settle for an imperfect assessment which measures safety in a probabilistic sense (USNRC, 1991). In this respect performance assessment of LLW sites has some similarities to` risk analyses of nuclear power plants, manned spacecraft, and other complex physical systems. There are, however, some important differences. The performance of a waste disposal site is highly dependent on the way that the natural environment responds to uncertain external disturbances. The "natural barriers system" at such a site is not as well understood as manmade systems which are built to specification. Natural systems are difficult to monitor and control and waste disposal performance assessments typically require predictions to be made over long time scales. All of these factors suggest that performance assessments of disposal sites need to be handled differently than those of power plants and other manmade systems.

We have seen in Chapter 2 that the hydrogeological properties of natural environments can be very heterogeneous. Heterogeneities can occur over a wide range of scales (Gelhar, 1986). In arid environments anomalous events such as high intensity storms may affect moisture and solute transport in the unsaturated zone (Gee and Hillel, 1988). It seems reasonable to expect that our ability to predict exposure and other relevant measures of site performance will depend on the heterogeneity of the site, the nature of external disturbances, and the data available for performance assessment. A complete performance analysis should consider all of these factors in an integrated way.

In this Chapter we outline a stochastic approach to performance assessment which addresses some of the distinctive problems encountered in low-level waste disposal applications. We confine our attention to the characterization of the natural unsaturated subsurface environment (vadose zone) in the vicinity of a low-level waste disposal facility. This environment has an important influence on the waste isolation capabilities of facilities located at or near the ground surface in arid regions. Our emphasis on the unsaturated zone reflects the scope of our research and does not imply that this is the only important part of the "natural barriers" system. Other important transport pathways are discussed in performance assessment reviews such as Kozak et al. (1990).

The discussion which follows emphasizes the following aspects of performance assessment: 
1. Site Characterization -- Site characterization identifies the key physical and chemical processes which affect waste transport at the site of interest. Ideally, the characterization should suggest quantitative models which may be used to predict exposure levels and other variables needed to assess risk. It should also provide the data needed to estimate uncertain model parameters.

2. Model Validation -- Model validation checks to see whether the models developed during site characterization are consistent with available observations of the predicted variables. The criteria used to establish consistency are application-dependent but should, in any case, account for heterogeneity and uncertainty.

3. Uncertainty Evaluation -- Uncertainty evaluation provides quantitative estimates of the accuracy of model predictions over the time and space scales of interest in the performance assessment. If they are available, such estimates can be used to evaluate the risk that the site will not perform adequately.

It is useful to briefly consider how these three activities are related.

In performance assessment we are concerned with the uncertainty of long-term predictions (e.g. 100 years in low-level waste applications). Generally speaking, the time available for a performance assessment study is much less than the prediction horizon. For this reason we need to evaluate prediction accuracy before the fact, using uncertainty (or risk) analysis. The estimates of model uncertainty provided by risk analysis techniques are usually derived from the model under investigation (Kendall and Stuart, 1977). The confidence intervals of classical linear regression analysis assume, for example, that the variables in the regression model are linearly related (Draper and Smith, 1967). If the relationship between these variables is actually nonlinear, the confidence intervals do not adequately describe the model's predictive capabilities. Most risk analysis techniques only work when uncertainty is limited to the model's inputs (e.g. its constitutive parameters or source terms). If the model's basic structure is incorrect (i.e. if it cannot give accurate predictions for any input values) classical risk analysis techniques are not applicable (McLaughlin and Wood, 1988a,b).

The above discussion suggests that we need to establish whether or not a model has the correct structure before we can proceed with a quantitative analysis of prediction uncertainty. This is the purpose of model validation. A performance assessment should start with a site characterization program which produces scientifically credible models of the local environment. These models should be checked by comparing their predictions to available field measurements. Models which cannot be rejected can be assumed, at least tentatively, to have the proper structure. These "validated" models can then be used to evaluate the uncertainty of performance predictions. Although validation tests can never prove that a model is structurally correct they can screen out models which are clearly incompatible with available data. This lends more credibility to the performance assessment and increases the likelihood of obtaining legitimate estimates of prediction uncertainty. 
The ideas summarized above are developed further in the following sections. Our discussion of performance assessment is designed primarily to present general concepts and to indicate where more investigation is needed. Specific techniques are discussed in more detail in the cited references.

\subsection{Site Characterization}

Chapter 3 presents the equations which are generally used to describe the movement of fluids (e.g. water and air) and associated contaminants in the unsaturated zone. The applicability of these equations depends, to a large extent, on problem scale. In most low level waste applications we are interested in predictions of contaminant movement over spatial scales of hundreds of meters and over time scales of tens of years. Available field evidence suggests that the geohydrologic properties of the unsaturated zone can vary significantly over much smaller scales -- a few meters in the horizontal and even less in the vertical (see Chapter 2). Important sources of small to intermediate scale spatial heterogeneity include variations in soil texture and chemical composition, macropores of various sizes and shapes, relic stream channels, etc. Short-term spatial and temporal variations in precipitation can also be important if they are large enough to temporarily saturate macropores or to create localized areas of perched water. Since natural variability is significant over the modeling domains of interest in performance evaluation, the constitutive parameters and source terms which appear in the fundamental flow and transport equations will generally be time and/or location-dependent.

We cannot hope to adequately characterize all of the sources of heterogeneity mentioned above with available exploration technology. We can, however, hope to account for the large-scale effects of small-scale heterogeneities which recur in a more or less regular way. It seems reasonable to expect that large scale measures of waste migration such as travel times to site boundaries will be affected more by the aggregate effects of small scale variability than by any individual anomaly. This is, of course, a hypothesis that needs to be tested. But if it is true we can analyze waste disposal performance with large scale models that are based on so-called "effective parameters". The stochastic theory summarized in Chapter 2 shows how certain effective parameters (unsaturated hydraulic conductivity, moisture retention, and macrodispersivity) can be evaluated from field information. Predictions based on these parameters are intended to describe the aggregate (or large-scale) features of the subsurface flow and transport.

The modeling concepts outlined above have important implications for site characterization. These can be summarized as follows:

1. Scale Distinctions -- Stochastic methods provide a convenient way to derive largescale "average" behavior from small scale equations with variable paraneters. This is, however, feasible only if the site characterization process provides 1) information about large-scale hydrogeologic features and 2) information about small-scale variability (Rajaram and McLaughlin, 1990). Large-scale features include systematic changes in geological structure, trends, seasonal effects, etc. Such features can be described deterministically (e.g. in terms of large-scale changes in mean properties). Small-scale 
variability includes local variations in soil properties such as hydraulic conductivity and day-to-day variations in precipitation. Such features can be described probabilistically (e.g. in terms of covariance or spectral density functions).

2. Clustered Sampling -- Geological observations of the site and its surroundings can provide useful qualitative information about large-scale features. This generally needs to be supplemented by quantitative information obtained from soil samples and borehole measurements. The most efficient way to obtain both large and small-scale information from a field sampling program is to collect data in a number of widely-spaced clusters (Figure 4-1). Measurements from each cluster may be used to estimate soil property means and variances. Estimates from different clusters can be compared with hypothesis testing and analysis of variance techniques which are able to identify large-scale trends (see Scheffe, 1959). If a relatively large number of soil samples (e.g. greater than 20) are available from different depths within a single borehole it may be possible to get a rough estimate of the vertical correlation scales of relevant soil parameters. It may also be possible to obtain enough data to estimate horizontal correlation scales of readily measured variables such as porosity and saturated hydraulic conductivity. Correlation scale estimates will, however, be quite uncertain when derived from the limited amounts of data generally available (Kendall and Stuart, 1977). In practice, it may be better to infer correlation scales from qualitative geological information (e.g. lithology, soil texture, etc.). This possibility is discussed in detail in Gelhar (1993). In any case, uncertainties in soil property statistics will have an effect on the accuracy of predictions obtained from stochastic theories. The magnitude of this effect can be determined with the aid of sensitivity analysis (see Chapter 2).

3. Estimating Effective Parameters -- The statistics required to derive the effective parameters of large-scale stochastic models of unsaturated flow and transport depend on the particular parameterizations used to represent soil property functions such as moisture retention and unsaturated hydraulic conductivity (or relative permeability). The parameterization suggested in Chapter 2, as well as related parameterizations such as those used by Yeh et al. (1985a, b, c) and Mantoglou and Gelhar (1987a, b, c) emphasize the importance of the slope of the log hydraulic conductivity vs. tension curve (called in Chapter 2). The mean and variance of this slope provide important information about the textural (fine vs. coarse) variability of unsaturated soils and, as a result, they have a significant impact on the amount of horizontal spreading predicted by large-scale stochastic models. Estimates of $\alpha$ should be derived directly from laboratory measurements of log hydraulic conductivity over a reasonable range of tensions (up to hundreds of centimeters at arid sites), rather than indirectly from moisture retention curves. If possible, these slope estimates should be expressed as a function of tension (see Chapter 2). Moreover, the site characterization program should provide enough measurements to give at least a rough estimate of the variance in each sampling cluster. This issue deserves special mention since field investigations have not traditionally provided direct measurements of the unsaturated hydraulic conductivity function. Other important soil parameters include the saturated log hydraulic conductivity and the slope of the moisture retention curve (possibly expressed as a function of tension). Means, variances, and (possibly) correlation scales should be estimated for each of these 


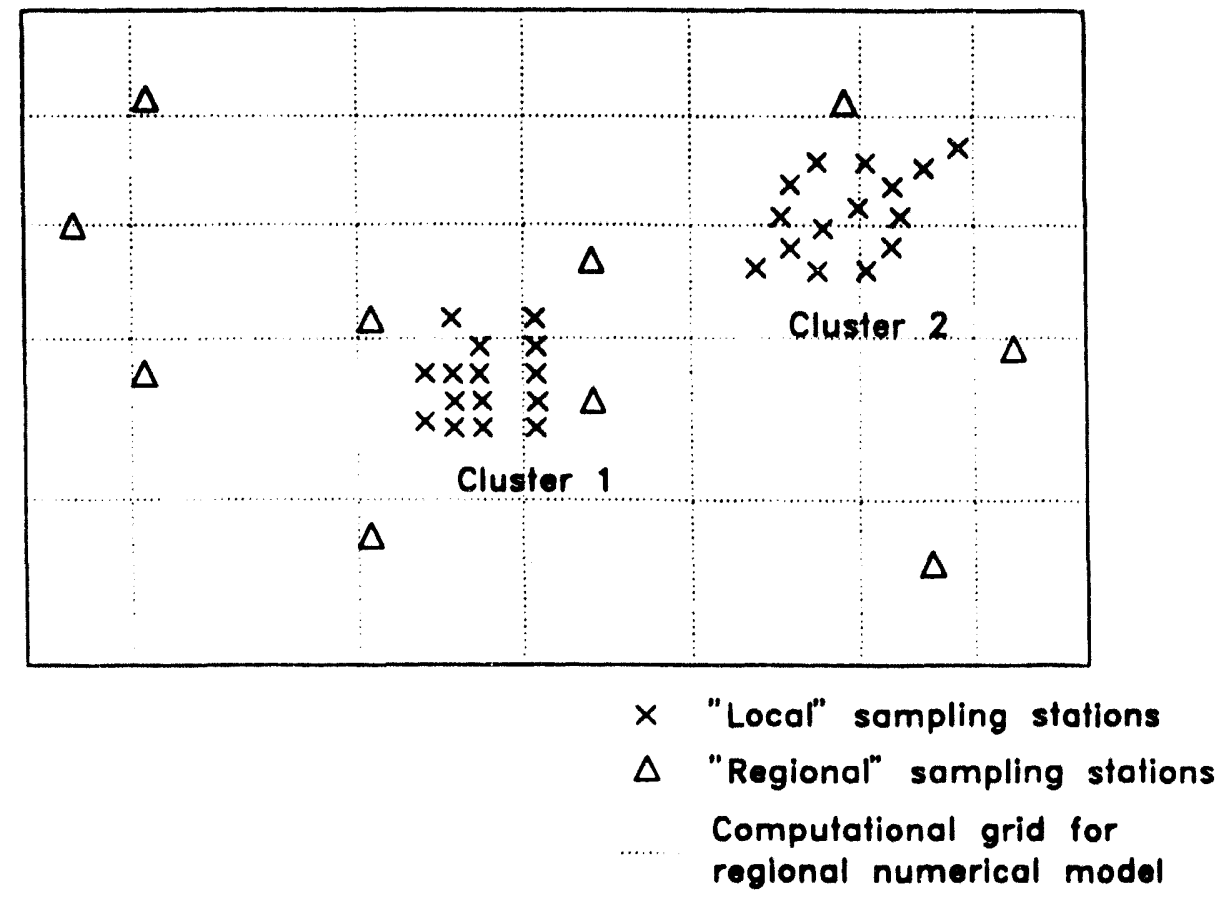

Figure 4-1. A clustered sampling strategy for site characterization (from Rajaram and McLaughlin, 1990). 
parameters in each sampling cluster. In addition, the correlation between $\alpha$, the saturated log conductivity, and the moisture retention slope should be estimated from soil samples where all these parameters are measured.

4. Site-Specific Sampling -- Particular care should 'e taken to tailor a site characterization effort to the unique features of each site. Generic site-independent characterization criteria are not necessarily more objective than customized sitedependent criteria. In fact, generalized criteria are more likely to miss critical features which may dominate waste transport. The real-world low-level waste disposal examples described in Bedinger and Stevens (1990) clearly reveal the importance of site-specific hydrogeological features. A site characterization effort should begin by identifying important transport pathways and a tentative set of related models. Subsequent data collection efforts should be designed 1) to serve the needs of the models and 2) to test the validity of model predictions. The particular models described in this report may be relevant to some sites (particularly sites where the unsaturated zone is an important transport pathway) but not to others. This question needs to be decided on a case-by-case basis.

If the above guidelines are followed, it should be possible to develop credible models of large-scale flow and transport in the vicinity of a low-level waste disposal site. Even large-scale models can be very computationally demanding to run when they are used to predict three-dimensional behavior over space scales of kilometers and time scales of tens of years. Recent improvements in numerical methods (see Chapter 3) and computer hardware have greatly expanded our ability to simulate large-scale unsaturated flow. But it should be recognized that practical licensing decisions made over the next several years may have to be based on simpler models which are even more aggregated than those discussed in this report. This topic is discussed further at the end of Section 4.4.

\subsection{Model Validation}

The problem of testing models to see whether or not they provide adequate descriptions of reality is as old as science itself. Over the years many investigators have pointed out that "model validation" efforts can never guarantee that the model in question is correct (see Konikow and Bredehoeft, 1992, for a recent discussion of this point). At best, validation tests can reject inadequate models or, more specifically, indicate where models fail to meet reasonable performance expectations. The topic of model validation is a venerable part of classical statistics, which is largely concerned with methods for testing various hypotheses (Scheffe, 1959). Classical statistical tests have not, however, typically been applied to hydrogeologic models such as those described in this report. This is primarily because such models are much more difficult to analyze than the simpler algebraic models discussed in most statistics texts.

Fortunately, the stochastic theory outlined in Chapter 2 and in the cited references provides enough information to apply classical hypothesis testing theory to the problem of validating large-scale models of low-level waste migration. This section describes a preliminary application of hypothesis testing techniques to two unsaturated flow models, 
one based on classical deterministic concepts and one based on effective parameters derived from stochastic concepts. Our model validation example relies on data collected at the Las Cruces, New Mexico field site mentioned in Chapter 2 (Wierenga et al., 1991). This example covers a more limited range of space and time scales (roughly 20 meters over two years) than a real performance assessment application. Nevertheless, it illustrates our approach as well as some of the important research issues that arise in model validation. The discussion which follows includes a summary of basic concepts, brief descriptions of the models investigated, and a review of important results.

\subsubsection{Basic Concepts}

The models produced by a practical site characterization effort will inevitably describe large-scale behavior, whether or not this is explicitly stated. Depending on the modeler's point-of-view, the model predictions may be interpreted as ensemble means, large-scale trends, or averages over space and time. The measurements available for testing such models are generally also averaged, but over much smaller space and time scales. So, for example, we may wish to test a model of large-scale unsaturated flow by comparing its predictions of moisture content to borehole measurements obtained from a neutron probe. The measurements of moisture content are averaged over a spatial scale of a few centimeters while the large-scale predictions of the model are most likely to apply over scales of tens of meters or more. These scale differences need to be accounted for when evaluating a model's performance.

If we follow the approach used in Chapter 2 and suppose that small-scale heterogeneity may be characterized stochastically, the ensemble mean of the predicted variable provides a convenient measure of large-scale behavior. In this case we can develop a quantitative analysis of the differences between small-scale measurements and large-scale model predictions. Consider, for example, a large-scale moisture content prediction $\sigma_{i}$, evaluated at location $x_{i}$ and time $t$ which is compared to a small-scale neutron probe measurement $\sigma_{i}{ }^{*}$ taken at the same location and time. The measurement residual $\varepsilon_{i}$ may be written as:

$$
\begin{aligned}
& \varepsilon_{i}^{*}=\theta_{i}^{*}-\hat{\theta}_{i}(\hat{\eta})=\left[\theta_{i}^{*}-\theta_{i}\right]+\left[\theta_{i}-\bar{\theta}_{i}\right]+\left[\bar{\theta}_{i}-\hat{\theta}_{i}(\eta)\right]+\left[\hat{\theta}_{i}(\eta)-\hat{\theta}_{i}(\hat{\eta})\right] \\
& \text { meas. error small-scale structural input error } \\
& \text { variability error }
\end{aligned}
$$

where $\theta_{i}$ is the true moisture content at $\left(x_{i}, t_{i}\right), \bar{\theta}_{i}$ is the mean moisture content (interpreted here to be the large-scale component of the local moisture content), $\eta$ is a vector of true model input values, and $\hat{\eta}$ is a vector of estimated model inputs. The variables $\hat{\theta}(. \eta)$ and $\hat{\theta}(\hat{\eta})$ are the model predictions obtained from $\eta$ and $\hat{\eta}$, respectively.

Equation [4-1] clearly identifies the various factors which contribute to observed differences between measurements and model predictions:

i) Measurement Error -- Differences between the measured and true small-scale values of moisture content. 
ii) Small-Scale Variability -- Differences between the large-scale trend we wish to predict and the true small-scale values of moisture content.

iii) Structural Error -- Differences between the large-scale mean and the model prediction obtained with perfect inputs.

iv) Input Estimation Error -- Differences between the model prediction obtained with perfect inputs and the prediction obtained with inputs estimated from a limited amount of field data.

The magnitudes of measurement errors, small-scale fluctuations, and input estimation errors can be quantified in terms of their respective statistics (e.g. means and variances). These statistics can be estimated, at least roughly, given certain assumptions about field measurement procedures, natural variability, and the mcthods used to derive model inputs. Structural errors, on the other hand, are the result of approximations, omissions, and faulty assumptions which are difficult to quantify. Such errors may arise if we apply one or two-dimensional models to three-dimensional problems, if we assume that soil property curves are linear when they are actually nonlinear, or if we assume conservative transport in situations where sorption is important.

One of the primary objectives of model validation is to determine whether structural errors are negligible (see Section 4.1). Hypothesis testing theory provides a convenient way to check for structural errors. We start by posing the following null hypothesis:

$$
H_{0}: \text { Model structural errors are negligible and } \hat{\theta}_{i}(\eta)=\bar{\theta}_{i}, \quad \forall i
$$

If this hypothesis is correct we should be able to attribute deviations between measurements and predictions solely to measurement error, small-scale variability, and input estimation error. We can test the hypothesis by 1 ) assuming that structural errors are zero, 2) deriving the resulting residual error statistics (e.g. mean and variance) from [4-1], and 3) checking to see if these derived statistics are "close" to the corresponding sample statistics.

It is relatively easy to show that the mean of each measurement residual should be zero when $H_{0}$ is true if the measurements and model input estimates are unbiased (Luis and McLaughlin, 1992). The variance of the measurement residual can also be derived if we introduce some ardditional assumptions. If $H_{0}$ is true and we assume that measurement errors, small-scale fluctuations, and input estimation errors are mutually uncorrelated the measurement residual variance is:

$$
\sigma_{\varepsilon_{i}}^{2}=\sigma_{m_{i}}^{2}+\sigma_{s_{i}}^{2}+\sigma_{e_{i}}^{2}
$$

where $\sigma_{m_{i}}^{2}$ is the variance of measurement error $\left[\theta_{i}^{*}-\theta_{i}\right], \sigma_{s_{i}}^{2}$ is the variance of the smallscale fluctuation $\left[\theta_{i}-\bar{\theta}_{i}\right]$, and $\sigma_{e_{i}}^{2}$ is the variance of the input estimation error $[\hat{\theta}(\eta)-\hat{\theta}(\hat{\eta})]$. The assumption that different errors are uncorrelated is made primarily to 
facilitate our discussion of hypothesis testing concepts. It is not crucial to the approach and may be relaxed if necessary (Luis and McLaughlin, 1992).

We need quantitative estimates of the variances appearing on the right side of [4-3] before we can evaluate the measurement residual variance. In our example, the measurement error variance can be inferred from replicated neutron probe calibration tests. The small-scale moisture content variance can be inferred from the enhanced stochastic theory described in Chapter 2 if the site characterization program provides sufficient information on small-scale soil property statistics. Note that this does not imply that the model itself must be based on stochastic concepts. The stochastic theory is needed only to evaluate the effects of small-scale variability on measurement residuals.

In classical regression analysis the effects of input estimation errors are generally inferred from a model sensitivity analysis (Draper and Smith, 1967). Dettinger and Wilson (1981) have used a variant of this approach to estimate the $\sigma_{e_{i}}^{2}$ term in [4.3]. In our notation, their approximation is given by:

$$
\sigma_{\varepsilon_{i}}^{2} \cong\left[\frac{\partial \hat{\theta}_{i}(\hat{\eta})}{\partial \eta}\right] P_{\eta \eta}\left[\frac{\partial \hat{\theta}_{i}(\hat{\eta})}{\partial \eta}\right]^{T}
$$

where $\partial \hat{\theta}_{i}(\hat{\eta}) / \partial \eta$ is a vector of sensitivity derivatives of the moisture content at $\left(x_{i}, t_{i}\right)$ taken with respect to the input variable vector (evaluated at the estimated value $\hat{\eta}$ ) and $P_{\eta \eta}$ is the estimation error covariance associated with $\hat{\eta}$. The derivative vector is generally evaluated numerically.

The estimation error covariance matrix $P_{\eta \eta}$ may be obtained in a variety of ways, depending on the input estimation procedure. For example, if statistical techniques such as kriging or maximum likelihood are used to estimate model inputs from field observations it is usually possible to obtain an approximate expression for $P_{\eta \eta}$ directly from the estimation algorithm (Schweppe, 1973; Kendall and Stuart, 1977; Journel and Huijbreghts, 1978). Monte Carlo techniques also provide a way to evaluate the effect of input estimation errors.

It should be pointed out that all available methods for evaluating the input estimation error variance component of [4-3] are computationally demanding when applied to the models described in Chapters 2 and 3. This is a major practical problem which needs to be resolved before we can derive accurate expressions for the measurement residual variance. Although it may be difficult to evaluate $\sigma_{\varepsilon_{i}}^{2}$ in practice we can obtain a conservative lower bound by assuming that the input estimation error variance $\sigma_{\varepsilon_{i}}^{2}$ is zero. Then all input estimation errors are interpreted as errors in model structure and variance-based hypothesis tests will be more inclined to reject $H_{0}$. This is the approach we have taken in the Las Cruces case study presented in Section 4.3.2.

When we have evaluated (or at least bounded) the measurement residual variance we may test the hypothesis that model structure errors are negligible. There are many possible tests which emphasize different types of structural error. The requirement that the 
measurement residuals have zero mean (when $H_{0}$ is true) can be checked with the following test:

Decide $H_{0}$ is true if: $m_{\varepsilon}=\left|\frac{1}{I} \sum_{i=1}^{l} \frac{\varepsilon_{i}}{\sigma_{\varepsilon_{i}}}\right|<v$

Reject $H_{0}$ otherwise

where $v$ is a test threshold selected to give a desired significance level (the "significance level" is the probability of rejecting $H_{0}$ when the hypothesis is true). If $H_{0}$ is true and the measurements are sufficiently far apart for the residuals to be uncorrelated, the normalized sample mean $m_{\varepsilon}$ will have a mean of zero and a standard deviation of 1 . If we assume that $m_{\varepsilon}$ is normally distributed (based on central limit considerations) the threshold value may be readily obtained from a standard normal probability table. If some of the measurements are too close for spatial correlations to be ignored, the test sample size $(I)$ may be reduced to account in an approximate way for correlation effects (Luis and McLaughlin, 1992).

The requirement that the measurement residuals have variances equal to the values derived from [4-3] (when $H_{0}$ is true) can be checked with the following test:

Decide $H_{0}$ is true if: $\chi^{2}=\sum_{i=1}^{l} \frac{\varepsilon_{i}^{2}}{\sigma_{\varepsilon_{i}}^{2}}<v$

Reject $H_{0}$ otherwise

If $H_{0}$ is true and the measurements are sufficiently far apart for the residuals to be uncorrelated normally distributed random variables, the test statistic $\chi^{2}$ follows a chisquared probability distribution with $I$ degrees of freedom. As with the mean test, the number of degrees of freedom may be reduced to account for correlation effects when the measurements are closely spaced.

The simple tests suggested above consider different aspects of the validation problem. The mean residual test checks for systematic biases while the mean-squared residual test checks for overall fit. These tests can be applied to all available measurements or to selected subsets such as all measurements taken at a particular time or along a particular transect. Moreover, these are not the only possible tests of mean shift and variance consistency. The range of possible testing alternatives complicates the task of reaching an unequivocal yes or no decision about the results of a model validation. Our view is that it is wise to carry out many different tests in order to establish an overall picture of model performance. This is illustrated in the example discussed in the next section.

\subsubsection{The Las Cruces Validation Study}

This section describes a relatively simple field application of the model validation approach outlined above. Our objective is to illustrate validation methodology rather than to advocate a particular model or modeling philosophy. The two models we consider both 
attempt to predict large-scale trends in moisture content. They differ only in the way that their effective properties are derived:

1) The Deterministic Model -- The effective soil properties (moisture retention and unsaturated log hydraulic conductivity curves) are assumed to equal the arithmetic averages of available measurements of the corresponding small-scale properties. The effective properties are assumed to be isotropic and to have the same functional form everywhere in the computational domain (see Figure 4-2).

2) The Stochastic Model -- Effective soil properties are derived from the enhanced stochastic theory of Chapter 2 . The resulting unsaturated hydraulic conductivities exhibit tension-dependent anisotropy but have the same basic functional form everywhere in the computational domain (see Figure 4-3).

Both models use the modified Picard numerical algorithm described in Chapter 3 to solve Richard's equation over the two-dimensional computational domain shown in Figure 2-5. Both sets of effective parameters are derived from laboratory analyses of approximately 450 soil cores taken from the north face of the Las Cruces trench site (Wierenga et al., 1989). The unsaturated log conductivity curves used to derive soil property statistics for the stochastic model are obtained indirectly from moisture retention curves, with the aid of the capillary bundle theory described by Mualem (1976) and van Genuchten (1980). It is likely that the resulting statistics underestimate the variability of the slope parameter $\alpha$, although it is difficult to know since unsaturated conductivity data are not available in the tension range observed over most of the site.

Our Las Cruces validation study tests each model's ability to predict about two years of neutron probe soil moisture measurements taken in a $14 \mathrm{~m}$ by $6 \mathrm{~m}$ vertical cross-section running parallel to the south side of the trench face. These measurements were obtained by sampling 8 neutron probe access tubes at $50 \mathrm{~cm}$ intervals in the vertical. Water was applied (at a rate of $1.8 \mathrm{~cm} /$ day for the first 82 days of the experiment) over a $4 \mathrm{~m}$ wide irrigation strip running perpendicular to the sampling cross-section. The adjacent ground surface was covered to impose a no-flux condition at the upper boundary of the computational domain (see Figure 2-5). Both models assume no flux conditions at the left (symmetry) and right boundaries. Measured initial values of volumetric moisture content ranged from about 5 to 10 percent, implying initial tensions greater than several thousand centimeters (see Figures 4-2 and 4-3).

Extensive information on our Las Cruces validation study is provided in Luis (1991) and in Luis and McLaughlin (1992). The validation procedure generally follows the approach described in the preceding section. Additional information on the field site is provided in Chapter 2 and in Wierenga et al. (1989), Nicholson et al. (1989), Nicholson (1990), and Luis and McLaughlin (1992). Since both models considered in our study depend on many assumptions (e.g. mean flow confined to a plane parallel to the trench face, initial and boundary conditions well known, effective parameters derived from simplified 

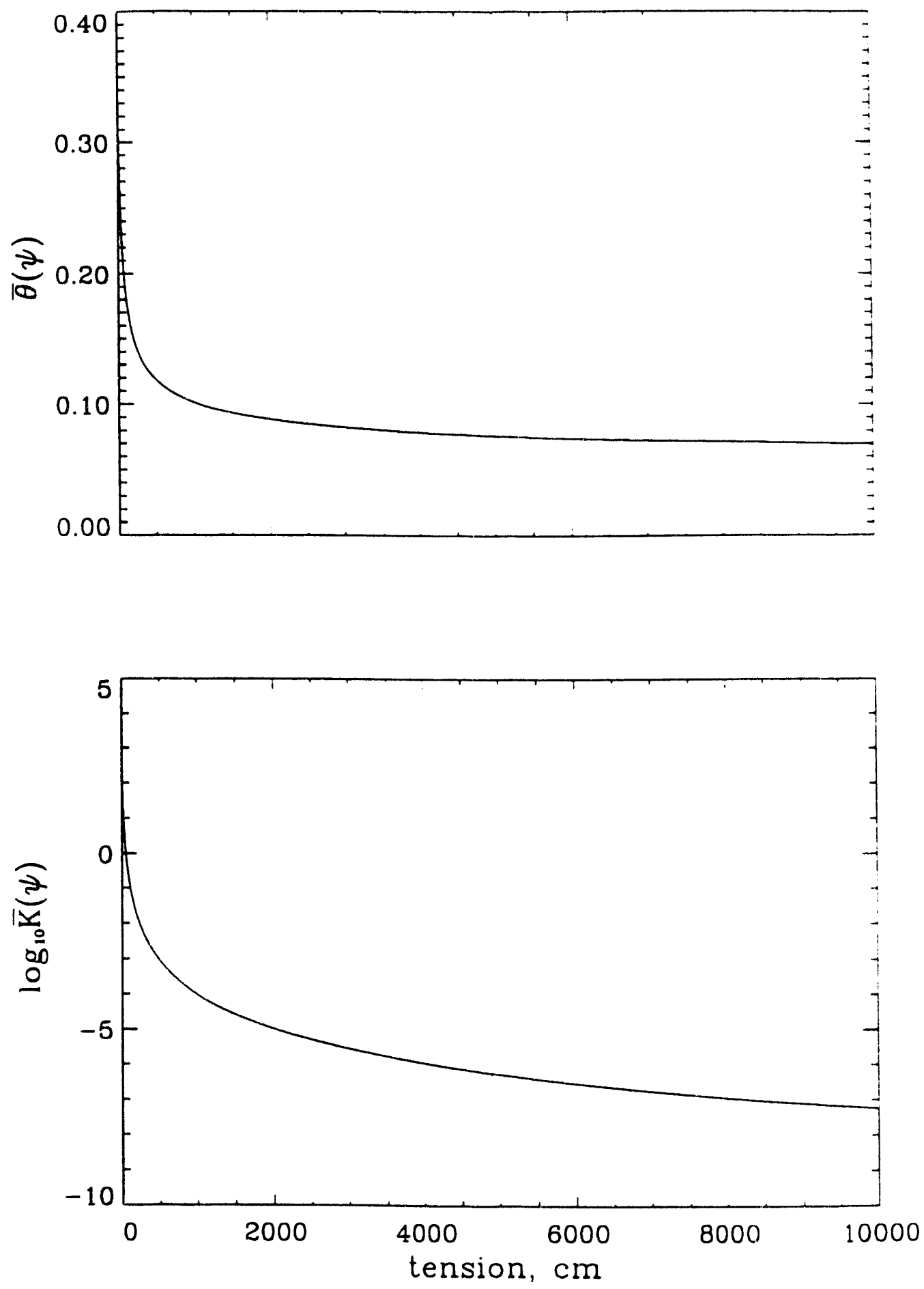

Figure 4-2. Effective parameters for the deterministic model. 


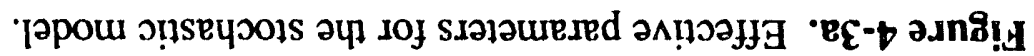

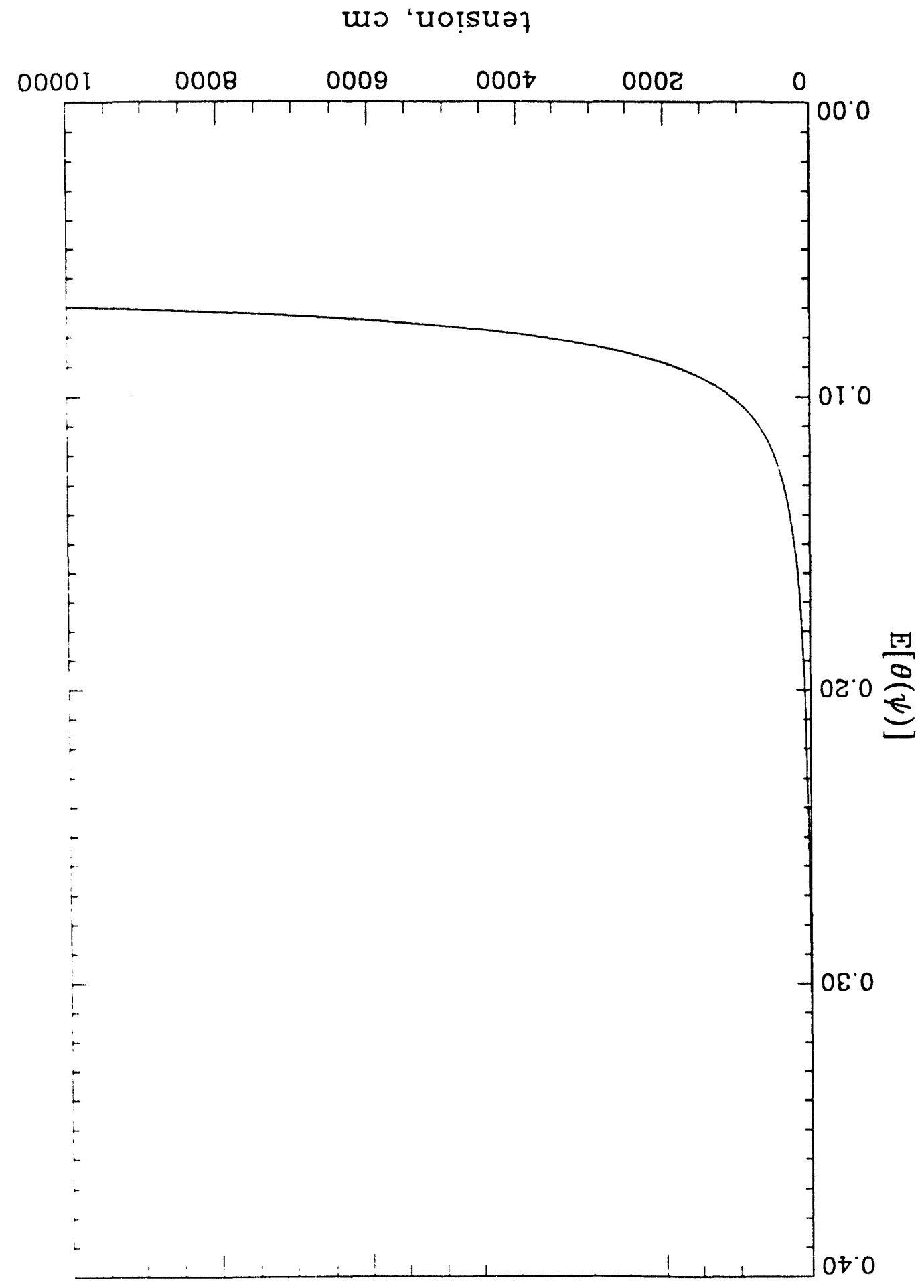



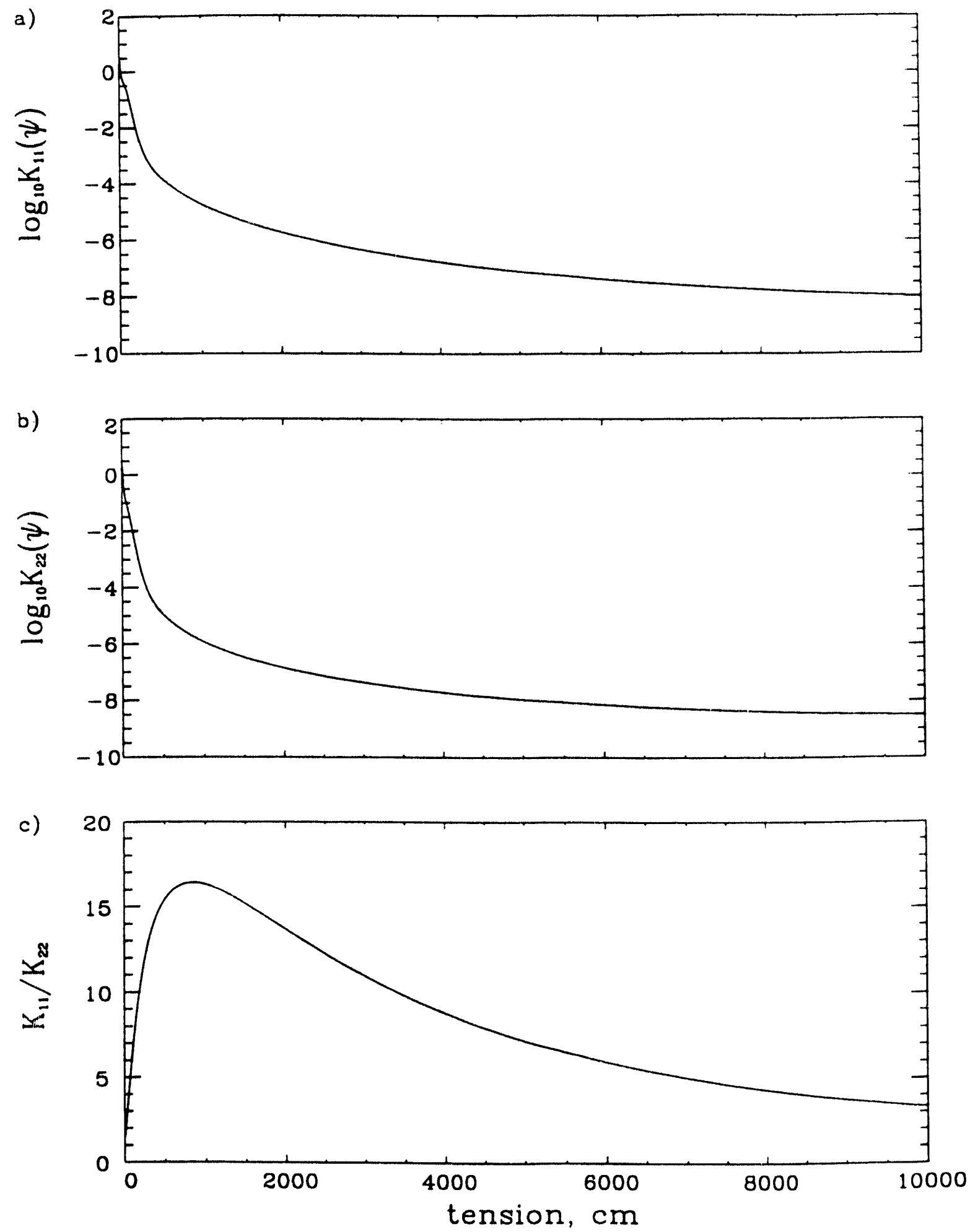

Figure 4-3b, c, d. Effective parameters for the stochastic model. 
expressions, etc.) and we neglect the effect of input estimation errors, it seems reasonable to expect that our hypothesis tests will find that structural errors are significant, at least at some times and locations.

Our Las Cruces validation study tests each model's ability to predict about two years of neutron probe soil moisture measurements taken in a $14 \mathrm{~m}$ by $6 \mathrm{~m}$ vertical cross-section running parallel to the south side of the trench face. These measurements were obtained by sampling 8 neutron probe access tubes at $50 \mathrm{~cm}$ intervals in the vertical. Water was applied (at a rate of $1.8 \mathrm{~cm} /$ day for the first 82 days of the experiment) over a $4 \mathrm{~m}$ wide irrigation strip running perpendicular to the sampling cross-section. The adjacent ground surface was covered to impose a no-flux condition at the upper boundary of the computational domain (see Figure 2-5). Both models assume no flux conditions at the left (symmetry) and right boundaries. Measured initial values of volumetric moisture content ranged from about 5 to 10 percent, implying initial tensions greater than several thousand centimeters (see Figures 4-2 and 4-3).

Extensive information on our Las Cruces validation study is provided in Luis (1991) and in Luis and McLaughlin (1992). Thie validation procedure generally follows the approach described in the preceding section. Additional information on the field site is provided in Chapter 2 and in Wierenga et al. (1989), Nicholson et al. (1989), Nicholson (1990), and Luis and McLaughlin (1992). Since both models considered in our study depend on many assumptions (e.g. mean flow confined to a plane parallel to the trench face, initial and boundary conditions well known, effective parameters derived from simplified expressions, etc.) and we neglect the effect of input estimation errors, it seems reasonable to expect that our hypothesis tests will find that structural errors are significant, at least at some times and locations.

It is useful to begin our discussion of validation results by comparing contour plots of measured and predicted moisture content values for Las Cruces Experiment 1. The plots obtained 82 days and 737 days after the start of the experiment are shown in Figures 4-4 and 4-5 respectively. Note that the computational domain extends well below the region plotted in these figures. This reduces the effect of the constant (ambient) tension condition imposed at the lower computational boundary. Tick marks around the borders of the plots indicate the node spacing used in the numerical simulation grid. A kriging algorithm is used to interpolate experimental data onto the same grid (Journel and Huijbreghts, 1978). It should be kept in mind that the outermost neutron tube boreholes are located at $x=-8.11 \mathrm{~m}$ and $+5.75 \mathrm{~m}$. Data values from these boreholes are extrapolated out to the $-10 \mathrm{~m}$ and $+10 \mathrm{~m}$ boundaries of the plots.

The smooth model predictions shown in Figures 4-6 and 4-7 differ substantially from the irregular experimental results. This effect can be seen to increase with time as the experimental moisture distribution shows a tendency to move laterally along preferred paths. By contrast, the predicted distributions quickly assume bulb-like shapes which persist throughout the simulation. Note that the stochastic model, with its anisotropic effective conductivity, predicts more horizontal spreading and slower vertical movement than the deterministic model. 

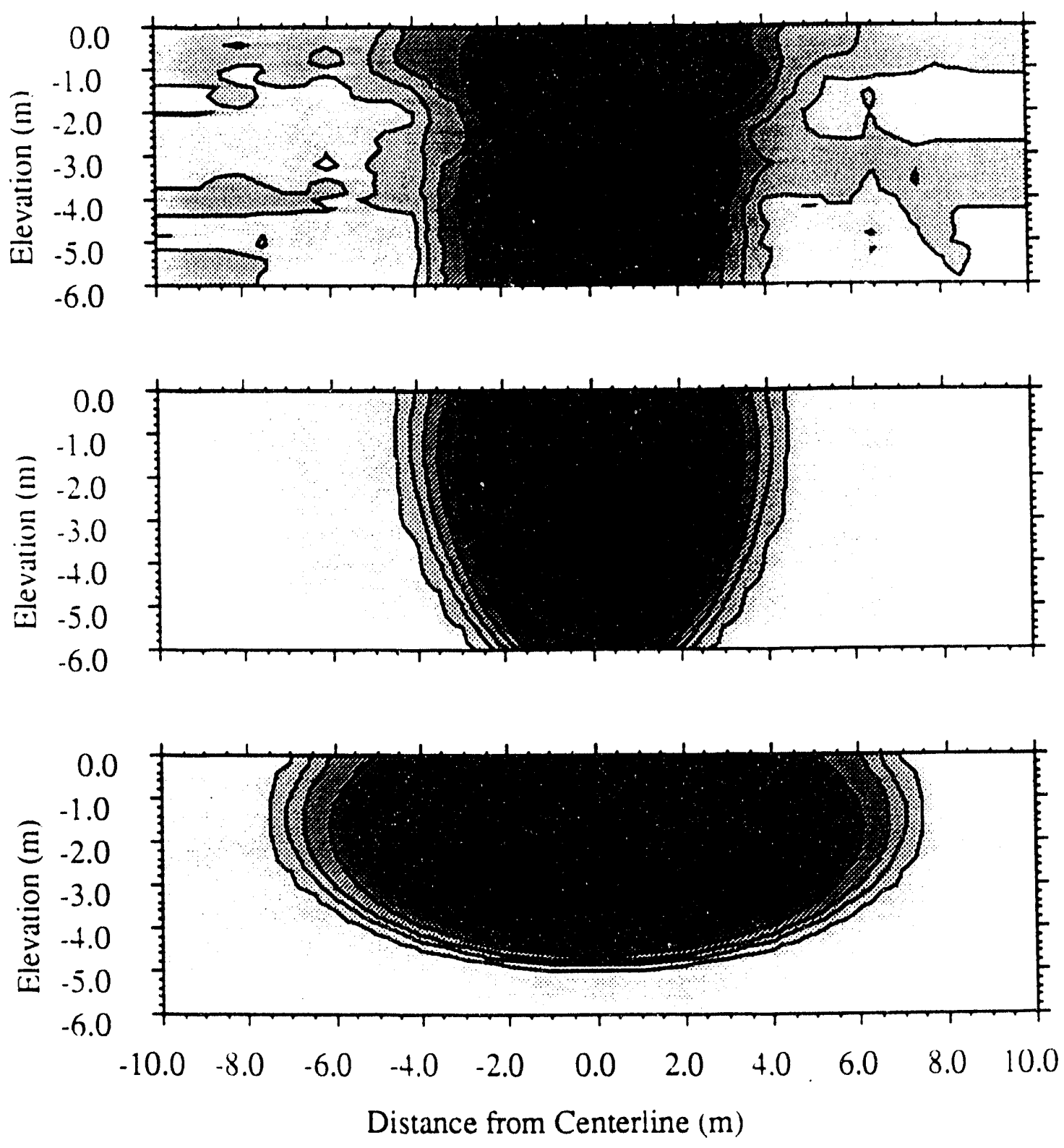



0.15 to 0.17

0.17 to 0.20

0.20 to 0.23

0.23 to 0.25

above 0.25

Figure 4-4. Las Cruces moisture content contours at 82 days. 

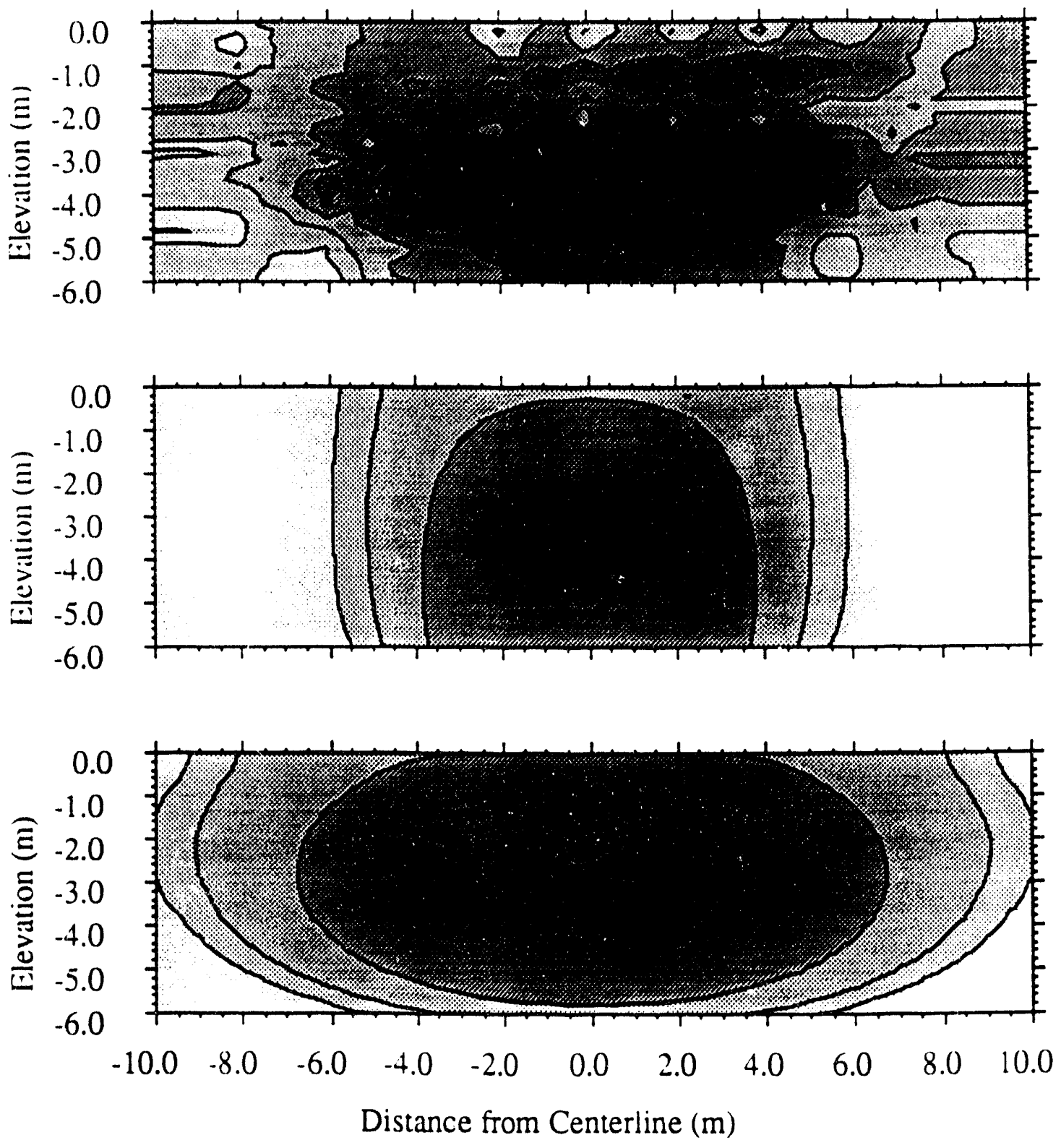

\begin{tabular}{l|l|l}
$\square$ below 0.05 & & 0.15 to 0.17 \\
$\square$ & 0.05 to 0.07 & 0.17 to 0.20 \\
0.20 to & 0.23 \\
0.07 to 0.10 & \\
0.10 to 0.12 & \\
0.23 to 0.25 \\
0.12 to 0.15 & & above 0.25
\end{tabular}

Figure 4-5. Las Cruces moisture content contours at 737 days. 


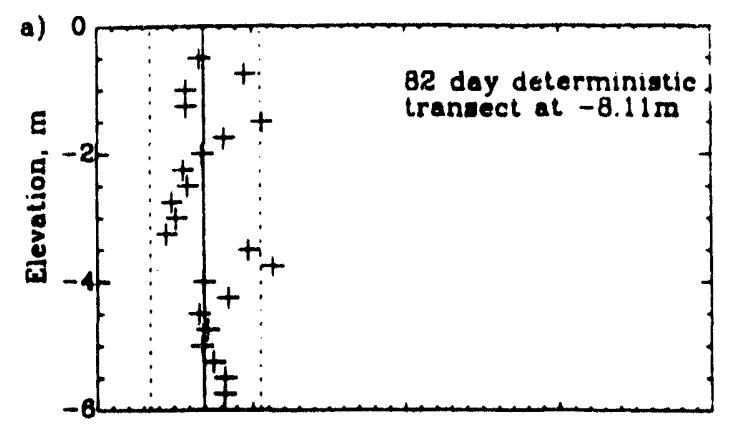

b)
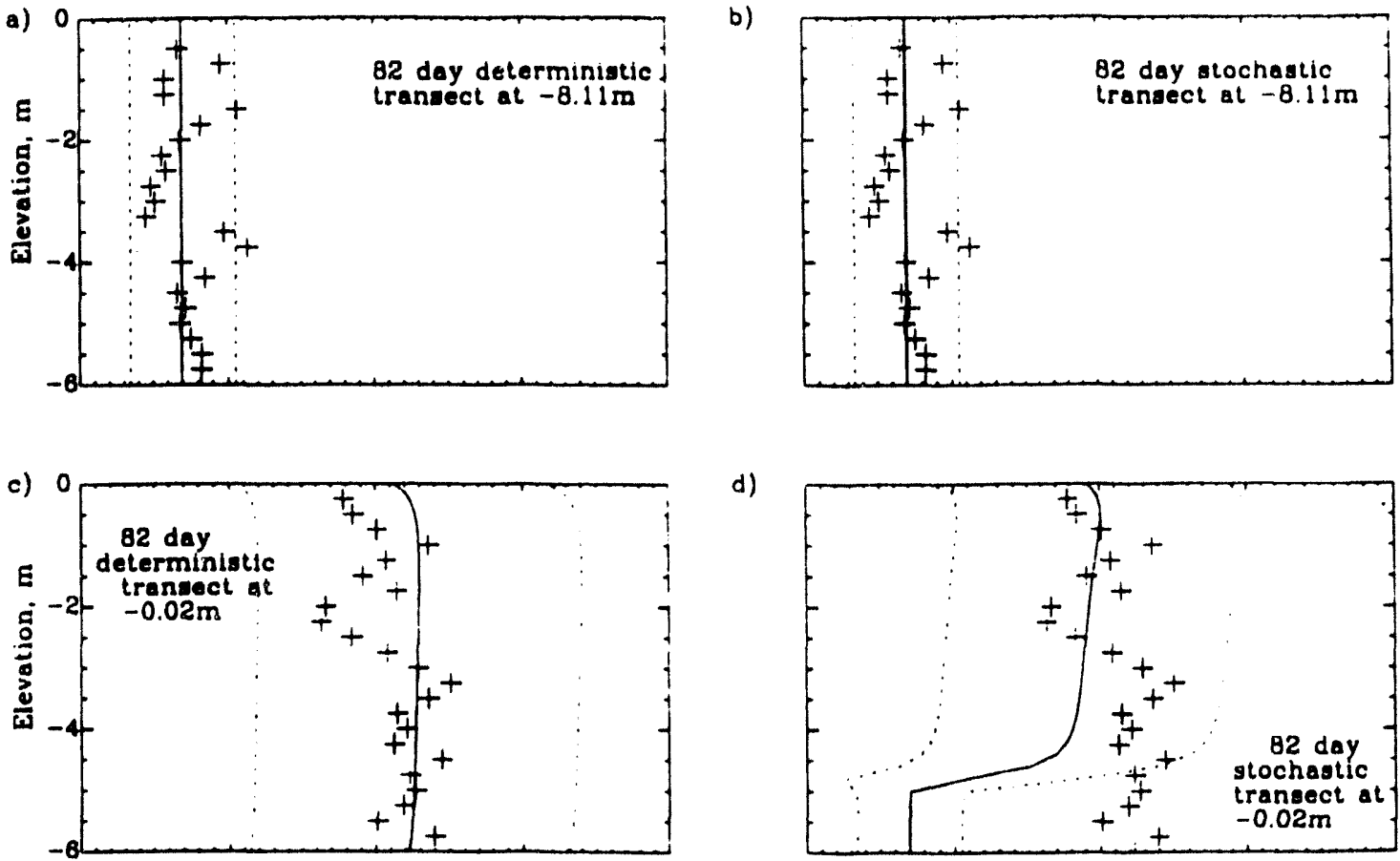

d)
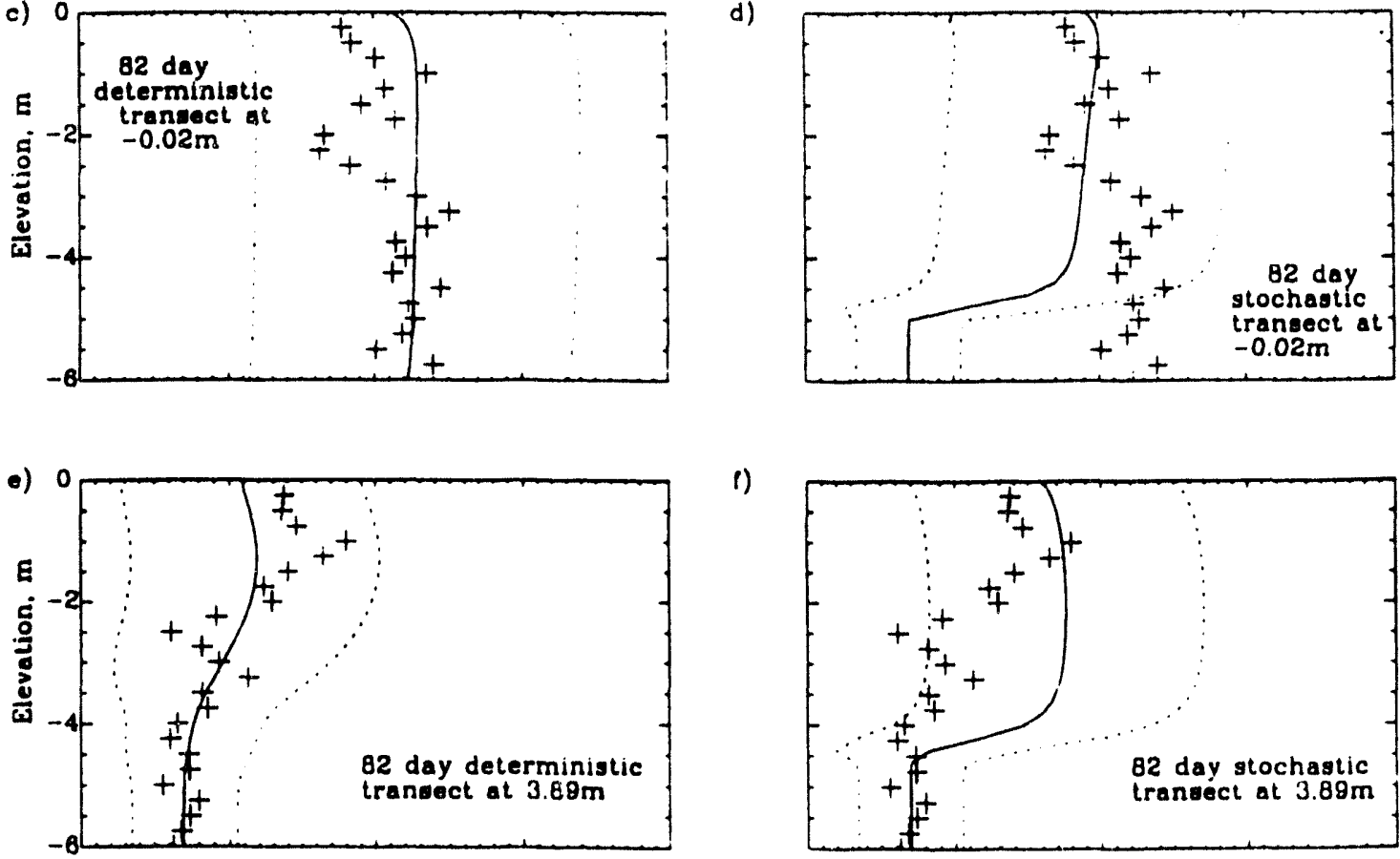

1)


Figure 4-6. Las Cruces vertical transects of moisture content at 82 days. 


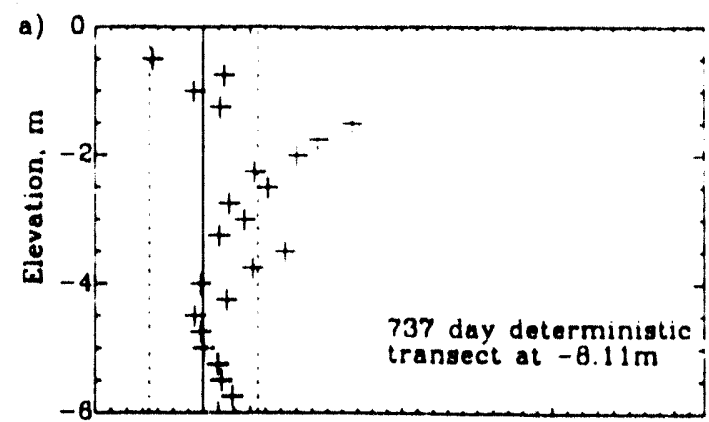

b)
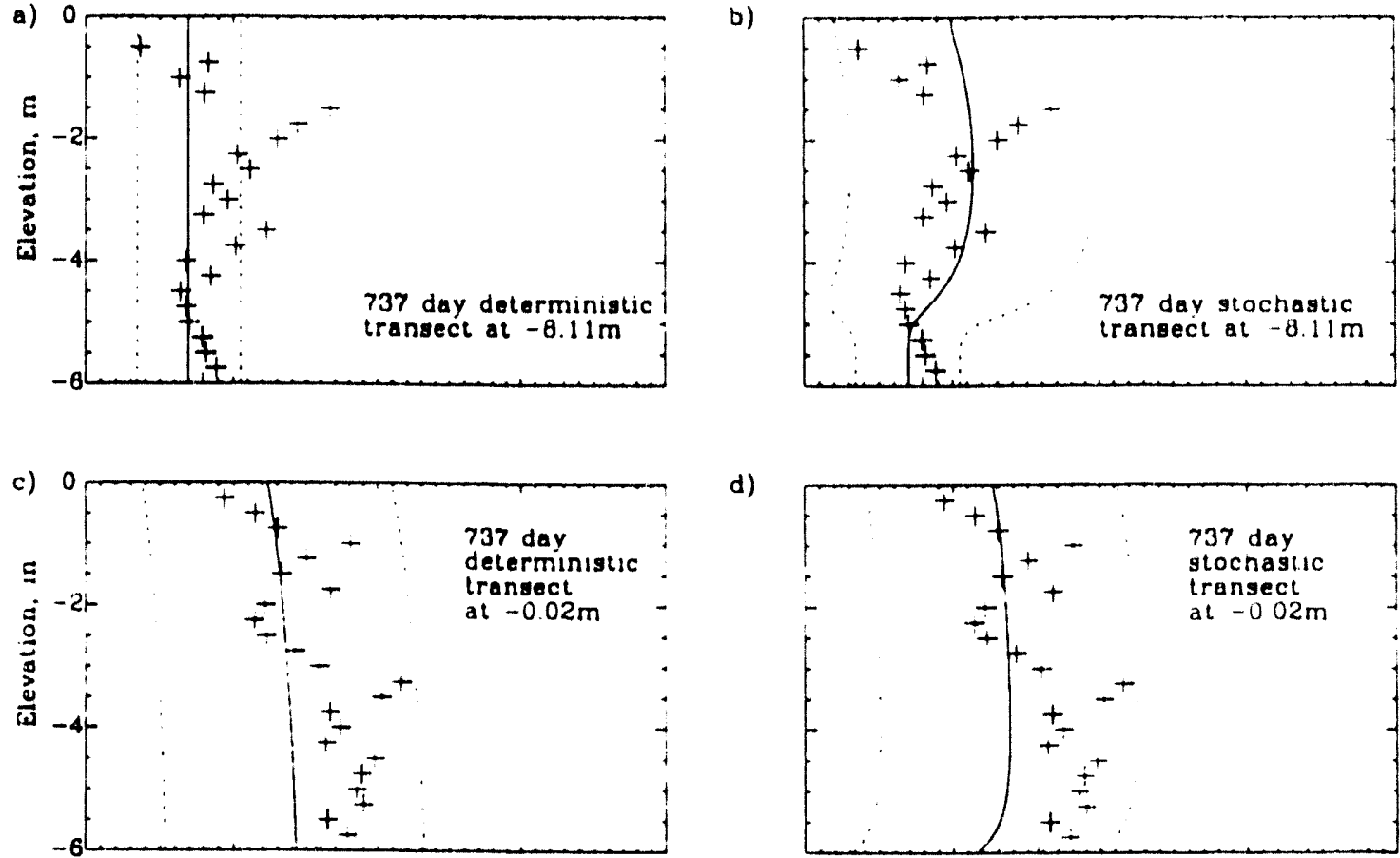

d)
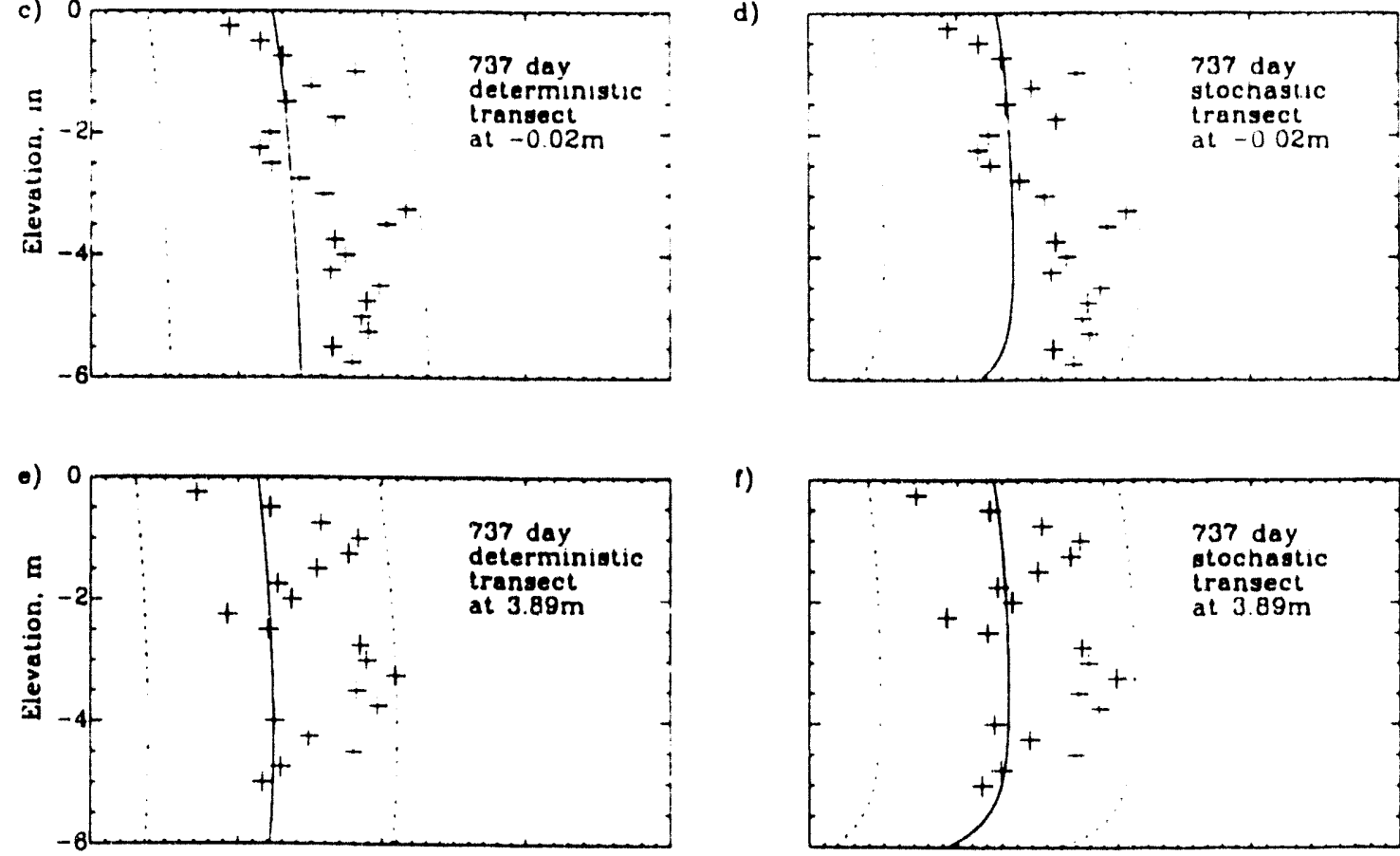

1)
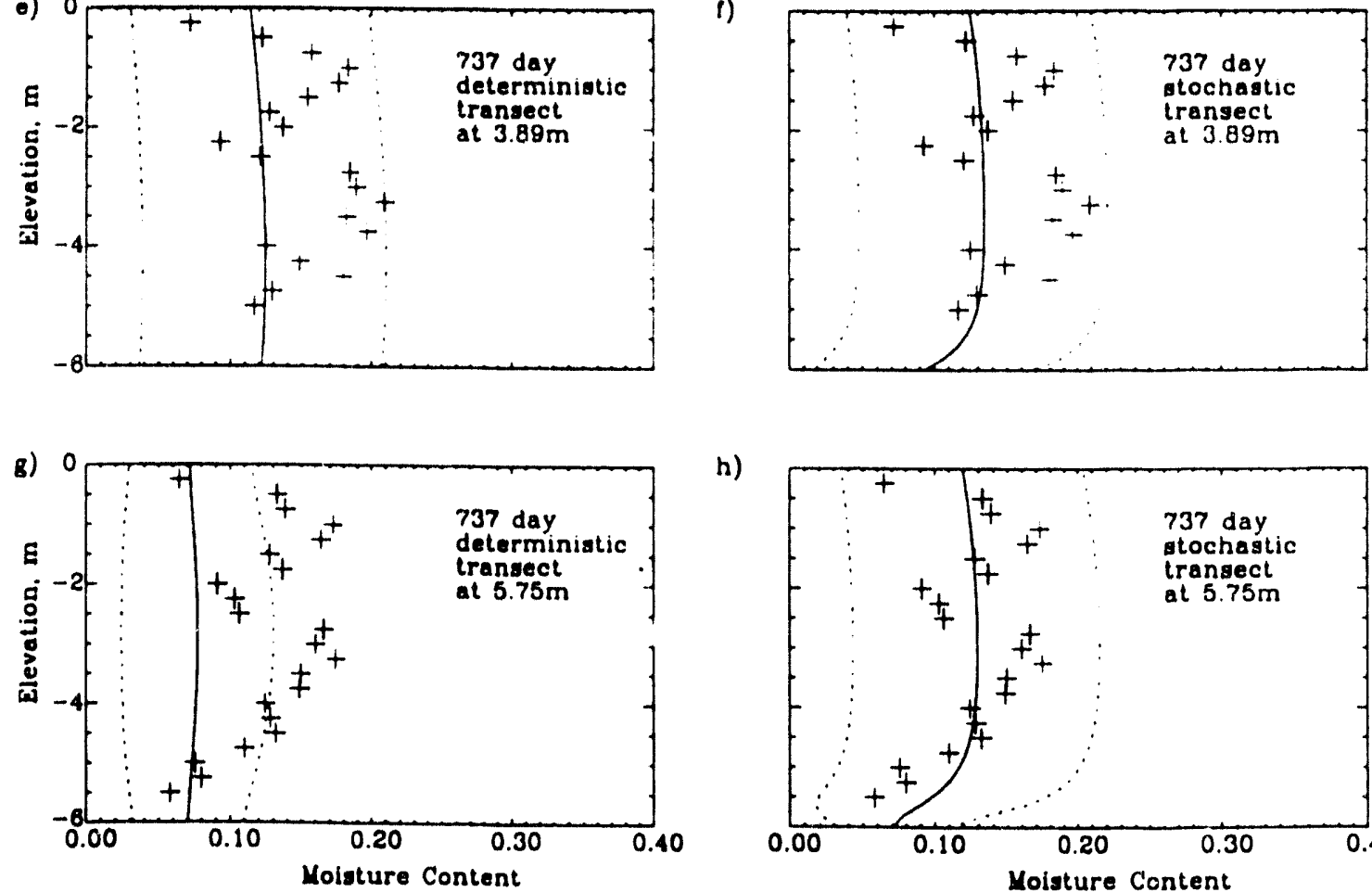

h)



Figure 4-7. Las Cruces vertical transects of moisture content at 737 days. 
It is possible to get a more spatially aggregated view of model performance if we compare the spatial moments of the measured and predicted moisture contents (Luis, 1991, Table 4-2). These moment comparisons generally confirm the qualitative differences in spreading and vertical infiltration which are revealed in the contour plots. They also suggest that the deterministic model matches the field observations somewhat better at 82 days while the stochastic model does better at 737 days.

Contour plots and spatial moment evaluations provide some indication of model capabilities but they do not compare the model prediction to the variable of primary interest here -- the mean moisture content. This can be done if we apply the error decomposition and hypothesis testing concepts described earlier. In particular, we can construct approximate confidence regions (based on a normality assumption) from the measurement residual variance of [4-3] and we can compute the test statistics and thresholds needed to carry out hypothesis tests. Figures 4-6 and 4-7 compare model predictions, $95 \%$ confidence regions and experimental data in selected neutron probe access tubes at 82 and 737 days, respectively. These results suggest that the deterministic model does better at early times while the stochastic model does better at later times (especially at the neutron probe sampling tubes furthest from the centerline).

The hypothesis tests of [4-5] and [4-6] provide additional insight about the model's behavior. Tables 1 through 3 (next page) show the results of the mean residual and meansquared residual tesis at a significance level of $5 \%$. Since the number of degrees of freedom (validation measurements) is reasonably large the test outcomes are nearly the same for all significance levels in the range from 1\% to 50\% (see Luis, 1991). The tests reported in Table 1 consider all locations simultaneously but are distinguish between sampling times. Those in Table 2 consider only the two outlying sampling tubes at -8.11 and $5.75 \mathrm{~m}$. Those in Table 3 consider only the centerline sampling tube. In each case, a "pass" means that the test accepts the Hypothesis $H_{0}$ (no model error) while a "fail" means that the test rejects this hypothesis.

The test results summarized in Tables $1-3$ confirm the general conclusions mentioned earlier -- the stochastic model does a better job at predicting long-term horizontal spreading while the deterministic model does a better job of predicting short-term vertical infiltration. When all times and locations are considered simultaneously both models fail the mean and mean-squared residual tests. This is not surprising, considering that we have made conservative assumptions throughout (e.g. ignoring input estimation error and spatial correlation between neutron probe measurements) and that we are evaluating prediction performance in a single vertical plane rather than a larger three-dimensional region. Certainly, the results of the test raise questions about the validity of basing longterm performance assessments on the deterministic predictions, which seem to degrade over time. On the other hand, the results are too inconclusive to lend much credibility to the stochastic model. It would be useful to carry out additional tests using data collected at times greater than 737 days. 


\section{TABLE 1}

Hypothesis Tests Results Over All Locations at Specified Times (5\% Significance Level)

Time (days)

$$
\begin{gathered}
\text { Mean Residual } \\
\text { Test }
\end{gathered}
$$

Determ.
Mean-Squared Residual Test

Determ.
Stoch.

$\begin{array}{cllll}30 & \text { Fail } & \text { Fail } & \text { Fail } & \text { Fail } \\ 82 & \text { Pass } & \text { Fail } & \text { Pass } & \text { Fail } \\ 371 & \text { Fail } & \text { Pass } & \text { Fail } & \text { Pass } \\ 737 & \text { Fail } & \text { Fail } & \text { Fail } & \text { Pass }\end{array}$

\section{TABLE 2}

Hypothesis Tests Results at Outlying Boreholes at Specified Times (5\% Significance Level)

Time (days)

\begin{tabular}{lllll}
\multicolumn{2}{c}{ (5\% Significance Level) } \\
\multicolumn{2}{c}{$\begin{array}{c}\text { Mean Residual } \\
\text { Test }\end{array}$} & \multicolumn{2}{c}{$\begin{array}{c}\text { Mean-Squared Residual } \\
\text { Test }\end{array}$} \\
& Determ. & Stoch. & Determ. & Stoch. \\
& & & & \\
e (days) & & & & Pass \\
30 & Pass & Pass & Pass & Pass \\
82 & Pass & Pass & Fail & Pass \\
371 & Fail & Pass & Fail & Pass \\
737 & Fail & Pass & &
\end{tabular}

\section{TABLE 3}

Hypothesis Tests Results at Centerline Borehole at Specified Times
(5\% Significance Level)
Mean Residual
Test
Mean-Squared Residual
Test
Determ. Stoch.

Time (days)
30

82

371

737

$$
\begin{aligned}
& \text { Fail } \\
& \text { Pass } \\
& \text { Pass } \\
& \text { Fail }
\end{aligned}
$$

$$
\begin{gathered}
\text { Fail } \\
\text { Fail } \\
\text { Pass } \\
\text { Fail }
\end{gathered}
$$

Fail
Pass
Pass
Pass

Fail

Fail

Pass

Pass 
Although the validation study described here is based on real field data it should not necessarily be interpreted as a typical application. We have emphasized predictions of mean moisture content rather than variables such as travel time or solute concentration, which are of more direct concern in performance assessments. The time and space scales of the validation study are probably too small to test the predictive capabilities of most interest in a typical low-level waste application (e.g. we may not care if the stochastic model is unable to predict very early time behavior). Surface conditions at the Las Cruces site are more controlled than at actual disposal sites where temporally-varying precipitation and evapotranspiration rates complicate the modeling process. Finally, we cannot always expect to have soil property data sets as large as those available at the Las Cruces site. Many of these issues can be addressed from within the general validation framework we have proposed. We view the Las Cruces application as an encouraging but preliminary test of our approach.

\subsection{Uncertainty Evaluation}

The basic goal of uncertainty evaluation is to determine the accuracy of the model predictions used to assess site performance. If the model's predictions fall well within the limits which define "acceptable" performance, we have some basis for granting a license. This raises the difficult question of what constitutes "well within" performance limits. One solution to this dilemma is to adopt a probabilistic (or risk-oriented) approach. Risk is measured in terms of the probability that the actual exposure can exceed a stated threshold (see Figure 4-8). Although we would like the risk to be zero, this is clearly unrealistic. In the absence of perfect information about the site and about future disturbances we can never be absolutely certain that the exposure threshold will not be violated. If we specify some small but non-zero exceedance probability we are essentially defining what we mean by "well within the limits of acceptable performance". If a model's prediction uncertainty is high we need to allow for a large margin of error when evaluating site performance. Otherwise we will not be able to attain the desired exceedance probability. If the predictions are accurate the margin of error can be reduced (compare Figures 4-8a and 4-8b).

We can use an error decomposition similar to [4-1] to identify the factors which contribute to prediction uncertainty when structural errors are negligible. In this case the true value of a particular variable $u(x, t)$ (e.g. exposure) is related to the model prediction as follows:

$$
\begin{aligned}
& \qquad(x, t)=\hat{u}(\hat{\eta} ; x, t)+[u(x, t)-\bar{u}(x, t)]+[\hat{u}(\eta ; x, t)-\hat{u}(\hat{\eta} ; x, t))] \\
& \text { true value prediction small-scale variability input error }
\end{aligned}
$$

Note that we have assumed here that Hypothesis $H_{0}$ of [4-2] is true so that $\bar{u}(x, t)=\hat{u}(\eta ; x, t)$ for any location $x$ and time $t$. This leaves only two sources of prediction error -- small-scale variability and input estimation error. Although we can never know for sure if structural error can be neglected over the entire time and space horizon of a performance assessment we can still condition the uncertainty analysis on Hypothesis $H_{0}$. If the model validation process fails to reject $H_{0}$ this is a reasonable 

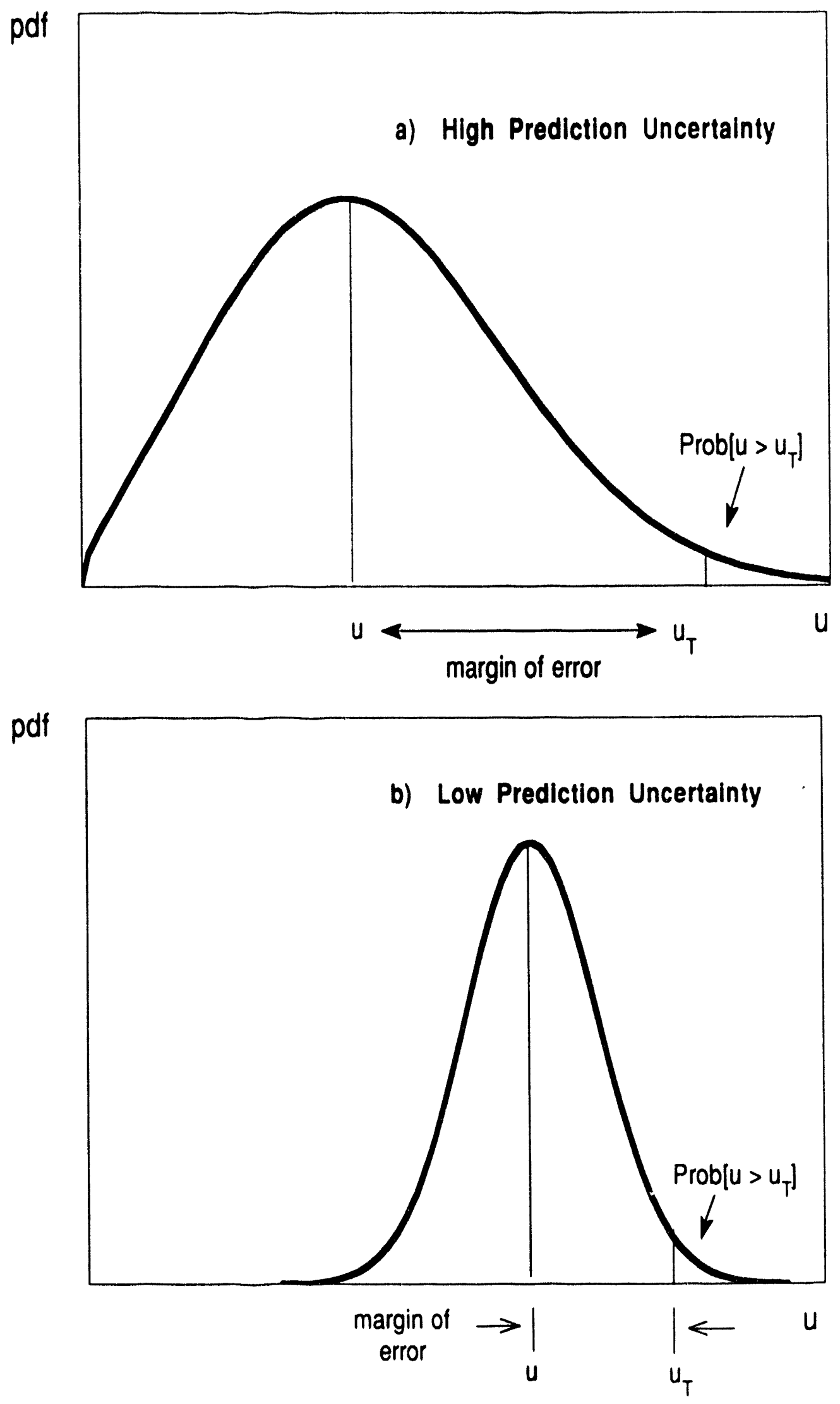

Figure 4-8. Margins of error for models with different prediction uncertainty $(u=$ exposure, $u_{T}=$ specified exposure threshold, $\hat{u}=$ model prediction) 
approach. If the validation rejects $H_{0}$ we have to improve the model before we can proceed with an uncertainty analysis based on [4.7].

The probability that the true exposure will exceed a given threshold can be derived from [4.7] if the joint probability density of the small-scale fluctuation and the input estimation error is known. The only general method available for deriving this density is the Monte Carlo simulation. Unfortunately, Monte Carlo methods are too computationally demanding to be applied to low-level waste performance analyses which rely on models as complex as those described in this report. Furthermore, the exceedance probability of interest in the performance assessment must be evaluated at the tail of the exposure distribution where Monte Carlo results are the least reliable.

For the reasons given above it seems unlikely that we can expect to carry out a probabilistic risk analysis which properly accounts for the complex physical processes which are known to occur at low-level disposal sites. We must either rely on very simple models which are amenable to Monte Carlo analysis but which may not pass validation tests or we must adopt a different approach to performance assessment. One plausible alternative is to simplify the relevant models as much as possible and then to use these models to derive rough bounds for prediction error. For example, approximate expressions for the variance of small-scale fluctuations in travel time or solute concentration could be derived from a version of the stochastic theory summarized in Chapter 2. Approximations similar to [4.4] could be used to evaluate the variance due to input estimation error. Although the resulting variances will not be as informative as the probabilities of exceedance required by a complete risk analysis they could give at least some indication of the margin of error needed in a given application.

The key to a scientifically credible, computationally feasible analysis of long-term site performance is the development of simplified models which are amenable to statistical analysis. These models should capture the physical processes that affect waste migration over distances of hundreds of meters and over time scales of tens of years. They will most likely achieve computational efficiency by sacrificing temporal and spatial resolution. This suggests that it may be more realistic to pose licensing regulations in terms of aggregated variables which are amenable to uncertainty analysis than in terms of point values. Although we can construct physically credible models which predict point quantities hundreds or even thousands of years into the future we cannot analyze the uncertainty of these predictions. As a result, the predictions have little relevance to the licensing process, which must account for risk.

The concepts introduced in the preceding paragraphs represent only a preliminary look at uncertainty evaluation. A number of other investigators have considered methods for evaluating the uncertainty of subsurface transport models (see, for example, Black and Freyberg, 1987; Graham and McLaughlin, 1988a, b; Vomvoris and Gelhar, 1990 and Li and McLaughlin, 1991). It seems fair to say that most of these studies are also preliminary. Nevertheless, recent progress by many researchers suggests that we are coming closer to the goal of accounting in a scientifically defensible way for natural variability and model uncertainty when assessing site performance. This is a long term 
effort which will have more impact on licensing decisions made in ten years than on those made in the next six months. In the meantime, regulators need to use common sense, remain sensitive to the need for good field data, and exercise healthy skepticism when using models to assess site performance. 


\section{CHAPTER 5}

\section{CONCLUSIONS}

This report summarizes recent enhancements and applications of MIT's stochastic approach for modeling contaminant transport in heterogeneous unsaturated soils. The stochastic viewpoint provides a convenient and realistic way to account for the complex soil variability found at typical low level radioactive waste disposal sites. It is important to account for such variability in modeling, site characterization, and performance assessment studies since small-scale heterogeneities can have a significant impact on the large-scale movement of moisture and associated contaminarits. This was shown in earlier studies (Yeh et al., 1985c; Mantoglou \& Gelhar, 1987c) which were based on simplified stochastic descriptions of soil variability.

The investigations described in this report extend earlier work by providing a more general framework for describing spatial variations in unsaturated log hydraulic conductivity and moisture retention functions. The generalized theory provides a pratical tool for estimating effective (or large-scale) soil properties for site-specific simulations models. In particular, the theory predicts that small-scale heterogeneity causes the effective log unsaturated hydraulic conductivity to exhibit tension-dependent aniostropy and hysteresis. It also predicts that the unsaturated longitudinal macrodispersivity will increase as the soil dries (i.e. will increase with increasing tension), reflecting the influence of small-scale velocity fluctuations. Finally, it predicts that the effective molecular diffusivity used to model large-scale diffusive transport of gaseous contaminants is significantly anisotropic in layered moist soils.

The effective parameters from the stochastic theory may be incorporated into models of unsaturated flow and transport for the water and vapor phases. The resulting model equations require numerical solution due to their high degree of nonlinearity. The project described here has been able to achieve significant improvements in numerical accuracy and efficiency by requiring numerical simulators to respect the physical principles (e.g. mass conservation) inherent in the governing equations. This makes it possible to carry out realistic simulations of systems with very dry initial conditions and high degrees of heterogeneity. Numerical simulation of the simultaneous movement of water and air in unsaturated soils has demonstrated the importance of coupling the air and wate phases in a more complete contaminated transport model.

Unsaturated flow and transport modeling can provide the basis for quantitative assessments of the performance of low-level waste sites. Model-based performance assessments should recognize the uncertainties associated with predictions based on simplyfying assumptions and limited data. The stochastic theory provides a systematic framework for evaluating such uncertainties. This report describes how stochastic techniques can be used to guide site characterization, model validation, and performance assessment efforts. Site characterization should provide information on the relative contributions of large and small-scale variability, preferably through the use of clustered 
sampling schemes. Particular attention should be given to the collection of data needed to compute the effective parameters used in stochastic flow and transport models.

Carefully designed site-specific sampling programs are to be preferred over programs based on generic guidelines.

Once a site has been adequately characterized and model predictions have been generated, the predictions need to be tested (compared with field data) to check that the model's assumptions are appropriate. The stochastic flow and transport theory can be combined with hypothesis testing concepts to give a systematic approach to model testing (or 'model validation'). This approach distinguishes errors in model structure from other sources of error and explicitly tests the hypothesis that the model's structure is correct. The Las Cruces example presented in this report shows that a hypothesis-based validation approach is feasible and informative. A stochastic model with no apparent structural errors may be used to establish confidence bounds on model predictions and to assess the probability that specified exposure standards will be violated. This systematic approach to performance assessment relies on the insights of the stochastic theory and on the efficiency and accuracy improvements provided by advanced numerical simulation techniques. It should prove useful in future model-based investigations of low level waste sites. 


\section{REFERENCES}

Ababou, R., L.W. Gelhar and D. McLaughlin, Three-dimensional Flow in Random Porous Media, Parsons Lab Report 318, R88-08, MIT, 1988.

Bedinger, M.S. and P.R. Stevens (Ed), Safe Disposal of Radionuclides in Low-Level Radioactive Waste Disposal Sites, Circular 1036, U.S. Geological Survey, Denver, 1990.

Bensabat, J., L.W. Gelhar and D.B. McLaughlin, "Stochastic Modeling of the First Las Cruces Trench Experiment", Appendix C, Las Cruces Trench Experiments in Voss, C.F. and T.J. Nicholsin, eds., Phase 1, Test Cases 10,11 and 12: Flow in Transport Experiments in Unsaturated Tuff and Soil, V-International INTRAVAL Project, Nuclear Energy Agency - OECD, Swedish Nulcear Power Inspectorate, Stockholm, Sweden, 1992.

Black, T.C. and D.L. Freyberg, Stochastic modeling of vertically averaged concentration uncertainty in a perfectly stratified aquifer, Water Resour. Res. 23(6):997-1004, 1987.

Bouloutas, E.B., "Improved Methods of Modeling Flow and Transport Processes in Partially Saturated Porous Media," PhD Thesis, Dept. of Civil and Env. Engg., MIT, 1989.

Bouloutas, E.T. and M.A. Celia, "Fast Iterative Solvers for Linear Systems Arising in the Numerical Solution of Unsaturated Flow Problems," to appear, Advances in Water Resources, 1992.

Celia, M.A., E.T. Bouloutas, and R.L. Zarba, " A general mass-conservative numerical solution for the unsaturated flow equation," Water Resour. Res. 26(7)1483-1496, 1990a.

Celia, M.A., T.F. Russell, I. Herrera, and R.E. Ewing, "An Eulerian-Lagrangian Localized Adjoint Method for the Advection-Diffusion Transport Equation," Advances in Water Resources, 13, 187-206, 1990b.

Celia, M.A., R.E. Ewing, and P. O'Leary, "Numerical Simulation of Unsaturated Flow and Contaminant Transport on a Massively Parallel Machine," presented at AGU Fall Meeting, 1991.

Celia, M.A. and P. Binning, "A Mass-Conservative Numerical Solution for Two-Phase Flow in Porous Media with Application to Unsaturated Flow", Water Resour. Res. 28(10), 2819-2828, 1992a.

Celia, M.A. and P. Binning, "Multiphase Models of Unsaturated Flow: Approaches to the Governing Equations and Numerical Methods," (Invited Paper), Proc. IX Int. Conf. on Computational Metnods in Water Resources, Vol. 2: Mathematical Modeling in Water Resources, Russell et al. (eds.), Elsevier, 257-272, 1992b. 
Celia, M.A., P. O'Leary, and R.E. Ewing, "Unsaturated Flow and Transport Simulation using PVM," to be submitted to Advances in Water Resources, 1992.

Cho, H.J., "The Volatilization of Organic Compounds in Unsaturated Porous Media during Infiltration," PhD Thesis, Dept. of Civil Engg., Princeton University, 1992.

Dagan, G., "Solute Transport in Heterogeneous Porous Formations, J. Fluid Mech. 145: $151-177,1984$.

Dettinger, M.D. and J.L. Wilson, First-order analysis of uncertainty in numerical models of groundwater flow, 1, Mathematical development, Water Resour. Res., 17(1):149-161, 1981 .

Ding, Y., Diffusive Transport of Gaseous Contaminants in Heterogeneous Unsaturated Soils, S.M. thesis, Parsons Lab, Dept. of Civil and Env. Engg., MIT, Cambridge, MA, 1992.

Draper, N.R. and M. Smith, Applied Regression Analysis, John Wiley, New York, 1967.

Ewing, R.E., T.F. Russell, and M.F. Wheeler, "Convergence Analysis of an Approximation of Miscible Displacement in Porous Media by Mixed Finite Elements and a Modified Method of Characteristics," Comp. Math. Appl. Mech. Engg., 47, 73-92, 1984.

Garabedian, S. P, L. W. Gelhar and M. A. Celia, "Large-Scale Dispersive Transport in Aquifers: Field Experiments and Reactive Transport Theory," Parsons Lab Rpt. 315, MIT, 1988.

Garabedian, S.P., D.R. LeBlanc, L.W. Gelhar and M.A. Celia, "Large-scale naturalgradient tracer test in sand and gravel, Cape Cod, Massachusetts, 2, Analysis of tracer moments for a nonreactive tracer", Water Resour. Res., 27(5):911-924, 1991.

Gee, G.W. and D. Hillel, Groundwater recharge in arid regions: Review and critique of estimation methods, Hydrological Processes, Vol. 2, 255-266, 1988.

Gelhar, L.W., "Stochastic subsurface hydrology from theory to applications," Water Resour. Res., 22(9): 135S-145S, 1986.

Gelhar, L.W., Stochastic Subsurface Hydrology, Prentice-Hall, Inc., 1993.

Gelhar, L.W. and C.L. Axness, "Three-dimensional stochastic analysis of macrodispersion in aquifers, Water Resour. Res. 19(1):161-180, 1983.

Gelhar, L.W., A. Mantoglou, C. Welty and K.R. Rehfeldt, "A Review Of Field Scale Physical Solute Transport Processes In Saturated And Unsaturated Porous Media," Final Rpt. RP2485-5, Elec. Power Res. Inst., Palo Alto, CA, EPRI EA-4190, August 1985. 
Gelhar, L.W., C. Welty and K.R. Rehfeldt, "A critical review of data on field-scale dispersion in aquifers", Water Resour. Res., 28(7):1958-1974, 1992.

Graham, W. and D. McLaughlin, Stochastic analysis of nonstationary subsurface solute transport, 1, Unconditional moments, Water Resour. Res., 25(2):215-233, 1989a.

Graham, W. and D. McLaughlin, Stochastic analysis of nonstationary subsurface solute transport, 2, Conditional moments, Water Resour. Res., 25(11):2331-2355, 1989.

Hills, R.G. and P.J. Wierenga, Model Validation at the Las Cruces Trench Site, Report NUREG/CR-5716, U.S. Nuclear Regulatory Commission, Washington, DC, 1991.

Journel, A.G. and Ch. I. Huijbreghts, Mining Geostatistics, Academic Press, San Diego, 1978.

Kendall, M. and A. Stuart, The Advanced Theory of Statistics, Vol. 2 Inference and Relationship, Macmillan, New York, 1977.

Kirkland, M.R., R.G. Hills, and P.J. Wierenga, "Algorithms for solving Richards equation in variably saturated soils," Water Resources Research, 28(8):2049-2058, 1992.

Konikow, L.F. and J.D. Bredehoeft, Groundwater models cannot be validated, Advances in Water Resources, 15, 75-83, 1992.

Kozak, M.W., M.S.Y. Chu and P.A. Mattingly, A Performance Assessment Methodology for Low-Level Waste Facilities, Report NUREG/CR-5532, U.S. Nuclear Regulatory Commission, Washington, DC, 1990

LeBlanc, D.R., D.L. Rudolph, R.G. Kachanoski, and M.A. Celia, "Design and Operation of an Infiltration Experiment in the Unsaturated Zone, Cape Cod, Massachusetts," in USGS Toxic Substance Hydrology Program: Proc. of the Technical Meeting, Mallard (ed.), Monterey, CA, 1991.

Li, S-G. and D. McLaughlin, A nonstationary spectral method for solving stochastic groundwater problems: Unconditional analysis, Water Resour. Res. 27(7):1589-1605, 1991.

Luis, S. J., A Stochastic Approach to Validation of Unsaturated Flow Models for Site Characterization 1.S. and Civil Engineer's thesis, Dept. of Civil and Env. Engg., MIT, August 1991.

Luis, S. and D. McLaughlin, A stochastic approach to model validation, Advances in Water Resources, 15, 15-32, 1992. 
Mantoglou, A, A theoretical approach for modeling unsaturated flow in spatially variable soils: Effective flow models in finite domains and nonstationarity, Water Resour. Res. 28(1): 251-267, 1992.

Mantoglou, A. and L.W. Gelhar, "Large-Scale Models of Transient Unsaturated Flow and Contaminant Transport Using Stochastic Methods", Report 299, Parsons Lab, MIT, Cambridge MA, 1985.

Mantoglou, A., and L. W. Gelhar, "Capillary tension head variance, mean soil content, and effective specific soil moisture capacity of transient unsaturated flow in stratified soils," Water Resour. Res., 23(1):47-56, 1987a.

Mantoglou, A., and L. W. Gelhar, "Effective Hydraulic Conductivities of Transient Unsaturated Flow in Stratified Soils," Water Resour. Res., 23(1):57-67, $1987 \mathrm{~b}$.

Mantoglou, A., and L. W. Gelhar, "Stochastic Modeling of Large-Scale Transient Unsaturated Flow Systeins," Water Resour. Res., 23(1):37-46, $1987 \mathrm{c}$.

McCord, J.T., D. B. Stephens, and J. L. Wilson, "Hysteresis and state-dependent anisotropy in modeling unsaturated hillslope hydrologic processes", Water Resour. Res. 27(7):1501-1518, 1991

McLaughlin, D. and E.F. Wood, A distributed parameter approach for evaluating the accuracy of groundwater predictions, 1, Theory, Water Resour. Res., 24(7):1037-1047, 1988a.

McLaughlin, D. and E.F. Wood, A distributed parameter approach for evaluating the accuracy of groundwater predictions, 2, Application to groundwater flow, Water Resour. Res., 24(7): 1048-1060, 1988b.

Mualem, Y., A new model for predicting the hydraulic conductivity of unsaturated porous media, Water Resour. Res., I2(3):513-522, 1976.

Nicholson, T.J., Recent Accomplishments in the INTRAVAL Project - A Status Report on Validation Efforts, Proc. Geoval 90 Symposium on Validation of Geosphere Flow and Transport Models, Stockholm, Sweden, May 14-17, 1990.

Nicholson, T.J., P.J. Wierenga, G. Gee, E.A. Jacobson, D.J. Polmann, D. McLaughlin, and L.W. Gelhar, Validation of Stochastic Flow and Transport Models for Unsaturated Soils: Field Study and Preliminary Results, Proc. DOE/AECL Conference on Cieostatistical, Sensitivity, and Uncertainty Methods for Groundwater Flow and Kadionuclide Transport Modeling, San Francisco, CA, Sept. 15-17, 1988, (CONF870971), Buxton, B.E. (Ed.), Battelle Press, Columbus, Ohio, pp. 275-296, 1989.

Office of the Federal Register, Code of Federal Regulations, Subparts C and D, 10, 672678, 1987. 
Polmann, D.J., E.G. Vomvoris, D. McLaughlin, E.M. Hammick and L.W. Gelhar, Application of Stochastic Methods to the Simulation of Large-Scale Unsaturated Flow and Transport, Report NUREG/CR-5094, U.S. Nuclear Regulatory Commission, Washington, DC, 1988.

Polmann, D.J., Application of Stochastic Methods to Transient Flow and Transport in Heterogeneous Unsaturated Soils, Ph.D. Thesis, Dept. of Civil and Env. Engg., MIT, Cambridge, MA, 1990.

Polmann, D. J., D. McLaughlin, L. W. Gelhar and R. Ababou, "Stochastic modeling of large-scale flow in heterogeneous unsaturated soils," Water Resour. Res., 27(7): 1447 $1458,1991$.

Rajaram, H. and D. McLaughlin, Identification of large-scale spatial trends in hydrologic data, Water Resour. Res., 26(10):2411-2423, 1990.

Robin, M.J.L., E.A. Sudicky, R.W. Gillham and R.G. Kachanoski, "Spatial variability of strontium distribution coefficients and their correlation with hydraulic conductivity in the Canadian forces base Borden aquifer", Water Resour. Res. 27(10):2619-2632, 1991.

Russo, D., "Stochastic analysis of simulated vadose zone solute transport in a vertical cross section of heterogeneous soil during nonsteady water flow", Water Resour. Res. 27(3):267-283, 1991.

Scheffe, H., The Analysis of Variance, John Wiley, New York, 1959.

Schweppe, F.C., Uncertain Dynamic Systems, Prentice-Hall, New Jersey, 1973.

Stephens, D.B. and S. Heermann, Dependence of anisotropy on saturation in a stratified sand, Water Resour. Res. 24(5):770-778, 1988.

Sudicky A, E., "A Natural Gradient Experiment on Solute Transport in a Sand Aquifer: Spatial Variability of Hydraulic Conductivity and Its Role in the Dispersion Process", Water Resour. Res., 22(13):2069-2082, 1986.

Sweed, H.G., P. Binning, and M.A. Celia, "Vapor-Phase Transport of Low Level Radioactive Waste in the Unsaturated Zone," Water Resources Program Report, Princeton University, 1992.

U.S. Nuclear Regulatory Commission, "Standard Review of Plan for the Review of a License Application for a Low-Level Radioactive Waste Disposal Facility", NUREG1200 Rev. 2, Jan. 1991.

van Genuchten, M.Th., "A Closed-Form Equation for Predicting the Hydraulic Conductivity of Unsaturated Soils, Soil Sci. Soc. America Journal, 44, 5:892-898, 1980. 
Vomvoris, E.G. and L.W. Gelhar, Stochastic analysis of the concentration variability in a three-dimensional heterogeneous aquifer, Water Resour. Res. 26(10):2541-2562, 1990.

Wierenga, P.J., L.W. Gelhar, C.S. Simmons, G.W. Gee, and T.J. Nicholson, Validation of Stochastic Flow and Transport Models for Unsaturated Soils: A Comprehensive Field Study, Report NUREG/CR-4622, U.S. Nuclear Regulatory Commission, Washington, DC, August, 1986.

Wierenga, P.J., D. Hudson, J. Vinson, M.Nash and A Toorman, Soil Physical Properties ai the Las Cruces Trench Site, Soil and Water Science Report No. 89-002, Universicy of Arizona, Tucson, 1989.

Wierenga, P.J., R.G. Hills, and D.B. Hudson, "The Las Cruces trench site: characterization, experimental results, and one-dimensional flow predictions," Water Resources Research, 27(10):2695-2706, 1991.

Yeh, T.C., L.W. Gelhar and A.L. Gutjahr, Stochastic analysis of unsaturated flow in heterogeneous soils, 1, Statistically isotropic media, Water Resour. Res., 21(4):447-456, 1985a.

Yeh, T.C., L.W. Gelhar and A.L. Gutjahr, Stochastic analysis of unsaturated flow in heterogeneous soils, 2, Statistically anisotropic media with variable $\alpha$, Water Resour. Res., 21(4):457-464, 1985b.

Yeh, T.C., L.W. Gelhar and A.L. Gutjahr, Stochastic analysis of unsaturated flow in heterogeneous soils, 3, Observations and applications, Water Resour. Res., 21 (4):465471, 1985 c. 


